\title{
Oxidative Addition of Aryl Tosylates to Palladium(0) and Coupling of Unactivated Aryl Tosylates at Room Temperature
}

Amy H. Roy and John F. Hartwig

Department of Chemistry, Yale University

P.O. Box 208107, New Haven, CT 06520-8107

\section{Supporting Information}

1. Synthetic procedures, spectral and analytical data, and kinetic data

2. References for synthetic procedures

3. X-ray crystal structure and data for 5

4. X-ray crystal structure and data for 6 
General Methods. ${ }^{1} \mathrm{H},{ }^{13} \mathrm{C}$, and ${ }^{31} \mathrm{P}\left\{{ }^{1} \mathrm{H}\right\}$ NMR spectra were recorded on a Bruker DPX 400 or $500 \mathrm{MHz}$ Spectrometer, General Electric QE $300 \mathrm{MHz}$ spectrometer, or a General Electric Omega 500 spectrometer with tetramethylsilane or residual protiated solvent as a reference. Elemental analyses were performed by Robertson Microlabs, Inc., Madison, NJ. All ${ }^{31} \mathrm{P}$ and ${ }^{13} \mathrm{C}$ NMR spectra were proton decoupled. GC and GC/MS analyses were conducted with an HP-1 methyl silicone column. Ether, toluene, tetrahydrofuran, benzene, and pentane were distilled from sodium/benzophenone. PPF- $t$-Bu (PPF- $t$-Bu = 1-diphenylphosphino-2-di- $t$ butylphosphinoethylferrocene) was purchased from Strem Chemicals and used without further purification. CyPF- $t$-Bu (CyPF- $t$-Bu $=1$-dicyclohexylphosphino-2-di- $t$ butylphosphinoethylferrocene) was obtained from Solvias AG and Strem Chemicals and used without further purification. $\left\{\mathrm{Pd}\left[\mathrm{P}(o \text {-tolyl })_{3}\right](\mathrm{Ph})(\mu-\mathrm{Br})\right\}_{2},{ }^{1} \mathrm{Pd}(\mathrm{dba})_{2},{ }^{2}$ and $\mathrm{Pd}\left[\mathrm{P}(o \text {-tolyl })_{3}\right]_{2}{ }^{1}$ were prepared by literature procedures.

Synthesis of Pd(PPF-t-Bu)[P(o-tolyl $\left.)_{3}\right]$ (1). A solution of $9.1 \mathrm{mg}(0.017 \mathrm{mmol})$ of PPF- $t$-Bu in $\mathrm{C}_{6} \mathrm{D}_{6}$ was added dropwise to a suspension of $12.0 \mathrm{mg}(0.0168 \mathrm{mmol})$ of $\mathrm{Pd}\left[\mathrm{P}(o \text {-tolyl })_{3}\right]_{2}$ in $\mathrm{C}_{6} \mathrm{D}_{6}$. The suspension was stirred at room temperature for $10 \mathrm{~min}$, during which time the solids dissolved. The solution was transferred to an NMR sample tube and was characterized by ${ }^{1} \mathrm{H}$ and ${ }^{31} \mathrm{P}\left\{{ }^{1} \mathrm{H}\right\}$ NMR spectroscopy. ${ }^{1} \mathrm{H}$ NMR $\left(\mathrm{C}_{6} \mathrm{D}_{6}\right) \delta 0.98(\mathrm{~d}, \mathrm{~J}=12.0 \mathrm{~Hz}, 9 \mathrm{H}), 1.24(\mathrm{~d}, \mathrm{~J}=11.2 \mathrm{~Hz}$, $9 \mathrm{H}), 1.86(\mathrm{t}, \mathrm{J}=6.4 \mathrm{~Hz}, 3 \mathrm{H}), 2.35\left(\mathrm{~s}, 9 \mathrm{H}\right.$, coordinated $\left.\mathrm{P}(o \text {-tolyl })_{3}\right), 3.32(\mathrm{~m}, 1 \mathrm{H}), 3.72(\mathrm{br} \mathrm{s}, 1 \mathrm{H})$, $3.86(\mathrm{~s}, 5 \mathrm{H}), 3.91(\mathrm{t}, \mathrm{J}=2.8 \mathrm{~Hz}, 1 \mathrm{H}), 4.18(\mathrm{br} \mathrm{s}, 1 \mathrm{H}), 6.87-7.18(\mathrm{~m}, 16 \mathrm{H}), 7.32(\mathrm{t}, \mathrm{J}=10.0 \mathrm{~Hz}, 2 \mathrm{H})$, 7.90 (br s, 2H), 7.99 (br t, J=8.0 Hz, 2H). ${ }^{31} \mathrm{P}\left\{{ }^{1} \mathrm{H}\right\} \mathrm{NMR}\left(\mathrm{C}_{6} \mathrm{D}_{6}\right) \delta 1.28$ (t, J=87.9 Hz), 19.78 (br $\mathrm{t}, \mathrm{J}=83.0 \mathrm{~Hz}), 71.69$ (br t, J=87.9 Hz). ${ }^{3}$

Synthesis of Pd(CyPF-t-Bu) $\left[\mathbf{P}(\boldsymbol{o} \text {-tolyl })_{3}\right]$ (2). A solution of $9.4 \mathrm{mg}(0.017 \mathrm{mmol})$ of CyPF- $t$-Bu in $\mathrm{C}_{6} \mathrm{D}_{6}$ was added dropwise to a suspension of $12.1 \mathrm{mg}(0.0169 \mathrm{mmol})$ of $\mathrm{Pd}\left[\mathrm{P}(o \text {-tolyl })_{3}\right]_{2}$ in $\mathrm{C}_{6} \mathrm{D}_{6}$. The suspension was stirred at room temperature for $10 \mathrm{~min}$, during which time the solids dissolved. The solution was transferred to an NMR sample tube and was characterized by ${ }^{1} \mathrm{H}$ and ${ }^{31} \mathrm{P}\left\{{ }^{1} \mathrm{H}\right\}$ NMR spectroscopy. ${ }^{1} \mathrm{H}$ NMR $\left(\mathrm{C}_{6} \mathrm{D}_{6}\right) \delta$ 0.90-2.0 (m, $\left.22 \mathrm{Cy} \mathrm{H}\right), 1.05$ (br d, J=8.8 Hz, $9 \mathrm{H}), 1.26$ (d, J=11.6 Hz, 9H), 1.88 (t, J=6.8 Hz, 3H), 2.2-2.6 (br, 9H), $3.15(\mathrm{~m}, 1 \mathrm{H}), 4.05$ (t, $\mathrm{J}=2.0 \mathrm{~Hz}, 1 \mathrm{H}), 4.13$ (s, 5H), 4.25 (br s, 1H), 4.36 (br s, 1H), 6.99-7.10 (m, 12H). ${ }^{31} \mathrm{P}\left\{{ }^{1} \mathrm{H}\right\} \mathrm{NMR}$ $\left(\mathrm{C}_{6} \mathrm{D}_{6}\right) \delta 11.72$ (br), 21.42 (br), $69.59(\mathrm{t}, \mathrm{J}=87.9 \mathrm{~Hz}){ }^{3}$

Independent Synthesis of Pd(PPF-t-Bu)(Ph)(OTs) (3). A solution of $200 \mathrm{mg}(0.240 \mathrm{mmol})$ of $\mathrm{Pd}(\mathrm{PPF}-t-\mathrm{Bu})(\mathrm{Ph})(\mathrm{Br})(5)$ in $\mathrm{C}_{6} \mathrm{H}_{6}$ was added to a suspension of $68 \mathrm{mg}(0.24 \mathrm{mmol})$ of AgOTs in $\mathrm{C}_{6} \mathrm{H}_{6}$. The reaction was complete after stirring for $20 \mathrm{~min}$, as determined by ${ }^{31} \mathrm{P}\left\{{ }^{1} \mathrm{H}\right\} \mathrm{NMR}$ spectroscopy. The suspension was filtered through Celite, and the orange solution was concentrated under vacuum. Addition of pentane and stirring of the resulting mixture led to 
precipitation of the product as an orange solid. The product was filtered, washed with pentane, and dried under vacuum to give $162 \mathrm{mg}$ (75\% yield) of product. ${ }^{1} \mathrm{H} \mathrm{NMR}\left(\mathrm{CD}_{2} \mathrm{Cl}_{2}\right) \delta 1.36(\mathrm{~d}$, $\mathrm{J}=13.2 \mathrm{~Hz}, 9 \mathrm{H}), 1.63$ (d, J=12.0 Hz, 9H), 2.01 (t, J=7.2 Hz, 3H), 2.21 (s, 3H), 3.28 (m, 1H), 3.69 (s, 5H), 4.10 (br s, 1H), 4.34 (br s, 1H), 4.61 (br s, 1H), 6.45 (br, 2H), 6.45 (t, J=7.2 Hz, 1H), 6.58 (br, 2H), 6.80 (d, J=8.4 Hz, 2H), 6.90 (d, J=7.6 Hz, 2H), 7.09-7.19 (m, 4H), 7.26-7.31 (m, $1 \mathrm{H}), 7.59-7.64(\mathrm{~m}, 3 \mathrm{H}), 8.17-8.22(\mathrm{~m}, 2 \mathrm{H}) .{ }^{13} \mathrm{C} \mathrm{NMR}\left(\mathrm{CD}_{2} \mathrm{Cl}_{2}, 40{ }^{\circ} \mathrm{C}\right) \delta 17.71(\mathrm{~d}, \mathrm{~J}=5.0 \mathrm{~Hz})$, 21.30, 31.64 (d, J=5.9 Hz), 31.70 (d, J=6.8 Hz), 32.43 (m), 37.47, 38.52, 68.62 (d, J=7.0 Hz), 70.88 (d, J=8.3 Hz), 70.95, 75.73 (d, J=5.2 Hz), 78.26 (dd, J=47.63, 10.80 Hz), 95.96 (dd, $\mathrm{J}=16.8,6.5 \mathrm{~Hz}), 123.22,126.40,126.77,128.04$ (d, J=11.3 Hz), 128.31, 128.77 (d, J=11.4 Hz), 129.92 ( J=2.8 Hz), 131.77 (br), 132.12 (d, J=58.1 Hz), 132.24 (d, J=58.1 Hz), 133.73 (d, J=9.6 $\mathrm{Hz}$ ), 136.02 (d, J=12.6 Hz), 136.67 (br), 139.05, 140.95, 157.83 (d, J=103.3 Hz). ${ }^{31} \mathrm{P}\left\{{ }^{1} \mathrm{H}\right\} \mathrm{NMR}$ $\left(\mathrm{CD}_{2} \mathrm{Cl}_{2}\right) \delta 25.4(\mathrm{~d}, \mathrm{~J}=37.4 \mathrm{~Hz}), 67.5(\mathrm{~d}, \mathrm{~J}=37.5 \mathrm{~Hz})$. Anal. calc'd for $\mathrm{C}_{45} \mathrm{H}_{52} \mathrm{P}_{2} \mathrm{O}_{3} \mathrm{SFePd}: \mathrm{C}$, 60.24; H, 5.85. Found: C, 59.94; H, 5.78.

Independent Synthesis of Pd(CyPF-t-Bu)(Ph)(OTs) (4). A solution of $113 \mathrm{mg}(0.140 \mathrm{mmol})$ of $\mathrm{Pd}(\mathrm{CyPF}-t-\mathrm{Bu})(\mathrm{Ph})(\mathrm{Br})(6)$ in $\mathrm{C}_{6} \mathrm{H}_{6}$ was added to a suspension of $48 \mathrm{mg}(0.17 \mathrm{mmol})$ of AgOTs in $\mathrm{C}_{6} \mathrm{H}_{6}$. The reaction was complete after stirring for $20 \mathrm{~min}$, as determined by ${ }^{31} \mathrm{P}\left\{{ }^{1} \mathrm{H}\right\}$ NMR spectroscopy. The suspension was filtered through Celite, and the orange solution was concentrated under vacuum. Addition of pentane and stirring of the resulting mixture led to precipitation of the product as an orange solid. The product was filtered, washed with pentane, and dried under vacuum to give $90 \mathrm{mg}$ (71\% yield) of product. ${ }^{1} \mathrm{H} \mathrm{NMR}\left(\mathrm{CD}_{2} \mathrm{Cl}_{2}\right)$ $\delta 0.76-2.95$ (m, 22 Cy H), 1.11 (d, J=13.5 Hz, 9H), 1.62 (d, J=12.0 Hz, 9H), 1.92 (t, J=8.0 Hz, $3 \mathrm{H}), 2.26(\mathrm{~s}, 3 \mathrm{H}), 3.02(\mathrm{~m}, 1 \mathrm{H}), 4.26(\mathrm{~s}, 5 \mathrm{H}), 4.52(\mathrm{t}, \mathrm{J}=2.0 \mathrm{~Hz}, 1 \mathrm{H}), 4.56$ (br s, 1H), 4.95 (br s, $1 \mathrm{H}), 6.79-6.84(\mathrm{~m}, 4 \mathrm{H}), 6.89(\mathrm{~d}, \mathrm{~J}=7.0 \mathrm{~Hz}, 2 \mathrm{H}), 7.05-7.12(\mathrm{~m}, 3 \mathrm{H}) .{ }^{31} \mathrm{P}\left\{{ }^{1} \mathrm{H}\right\} \mathrm{NMR}\left(\mathrm{C}_{7} \mathrm{H}_{8},-40{ }^{\circ} \mathrm{C}\right)$ $\delta 25.2(\mathrm{~d}, \mathrm{~J}=31.9 \mathrm{~Hz}), 71.6(\mathrm{~d}, \mathrm{~J}=30.5 \mathrm{~Hz})$. Anal. calc'd for $\mathrm{C}_{45} \mathrm{H}_{64} \mathrm{P}_{2} \mathrm{O}_{3} \mathrm{SFePd}$ : C, 59.44; H, 7.11. Found: C, 59.71; H, 7.34.

Independent Synthesis of Pd(PPF-t-Bu)(Ph)(Br) (5). A solution of $218 \mathrm{mg}(0.400 \mathrm{mmol})$ of PPF- $t$-Bu in THF was added dropwise to a suspension of $200 \mathrm{mg}(0.180 \mathrm{mmol})\{\mathrm{Pd}[\mathrm{P}(o-$ tolyl $\left.\left.)_{3}\right](\mathrm{Ph})(\mu-\mathrm{Br})\right\}_{2}$ in THF. The reaction mixture was stirred at room temperature for $20 \mathrm{~min}$, during which time all the solids dissolved. After 20 min the reaction was complete, as determined by ${ }^{31} \mathrm{P}\left\{{ }^{1} \mathrm{H}\right\}$ NMR spectroscopy. The orange solution was filtered through Celite and concentrated. Addition of pentane led to precipitation of $226 \mathrm{mg}$ (78\% yield) of the product as an orange solid. The product was filtered, washed with pentane, and dried under vacuum. ${ }^{1} \mathrm{H}$ $\operatorname{NMR}\left(\mathrm{CD}_{2} \mathrm{Cl}_{2}\right) \delta 1.39(\mathrm{~d}, \mathrm{~J}=12.8 \mathrm{~Hz}, 9 \mathrm{H}), 1.72(\mathrm{~d}, \mathrm{~J}=12.0 \mathrm{~Hz}, 9 \mathrm{H}), 2.07$ (t, J=8.0 Hz, 3H), 3.43 (m, 1H), $3.67(\mathrm{~s}, 5 \mathrm{H}), 4.22(\mathrm{~m}, 1 \mathrm{H}), 4.33(\mathrm{~m}, 1 \mathrm{H}), 4.59(\mathrm{~m}, 1 \mathrm{H}), 6.36-7.28(\mathrm{~m}, 10 \mathrm{H}), 7.60-7.66$ $(\mathrm{m}, 3 \mathrm{H}), 8.18-8.24(\mathrm{~m}, 2 \mathrm{H}) .{ }^{13} \mathrm{C} \mathrm{NMR}\left(\mathrm{CD}_{2} \mathrm{Cl}_{2},-15{ }^{\circ} \mathrm{C}\right) \delta 18.18(\mathrm{~d}, \mathrm{~J}=3.0 \mathrm{~Hz}), 31.05(\mathrm{~d}, \mathrm{~J}=4.3$ 
$\mathrm{Hz}$ ), 32.00 (br), 32.66 (dd, J=5.7, $3.1 \mathrm{~Hz}$ ), 37.48 (s at $25^{\circ} \mathrm{C}$, d with $\mathrm{J}=1.8 \mathrm{~Hz}$ at $-80{ }^{\circ} \mathrm{C}$ ), 38.86 , 67.92 (d, J=7.0 Hz), 70.20 (d, J=7.8 Hz), 70.42, 75.28 (d, J=4.7 Hz), 78.00 (dd, J=41.1, 8.7 Hz), 95.61 (dd, J=18.2, $6.3 \mathrm{~Hz}$ ), 121.07, 124.51 (d, J=7.5 Hz), 127.38 (d, J=12.1 Hz), 127.65 (d, J=7.0 $\mathrm{Hz}), 128.57$ (d, J=11.6 Hz), 128.74 (d, J=1.3 Hz), 131.33 (d, J=2.0 Hz), 131.80 (d, J=51.9 Hz), 132.49 (d, J=64.1 Hz), 132.61 (d, J=7.8 Hz), 133.33 (d, J=6.0 Hz), 136.23 (d, J=14.0 Hz), 139.56, $160.44(\mathrm{~d}, \mathrm{~J}=113.1 \mathrm{~Hz}) .{ }^{31} \mathrm{P}\left\{{ }^{1} \mathrm{H}\right\} \mathrm{NMR}\left(\mathrm{CD}_{2} \mathrm{Cl}_{2}\right) \delta 21.2(\mathrm{~d}, \mathrm{~J}=38.0 \mathrm{~Hz}), 67.6$ (d, J=38.0 Hz). Anal. calc'd for $\mathrm{C}_{38} \mathrm{H}_{45} \mathrm{P}_{2} \mathrm{BrFePd}$ : C, 56.63; H, 5.64. Found: C, 56.59; H, 5.49.

Independent Synthesis of Pd(CyPF-t-Bu)(Ph)(Br) (6). A solution of $157 \mathrm{mg}(0.28 \mathrm{mmol})$ CyPF- $t$-Bu in THF was added dropwise to a suspension of $150 \mathrm{mg}(0.13 \mathrm{mmol})\{\mathrm{Pd}[\mathrm{P}(o-$ tolyl $\left.\left.)_{3}\right](\mathrm{Ph})(\mu-\mathrm{Br})\right\}_{2}$ in THF. The reaction mixture was stirred at room temperature for $20 \mathrm{~min}$ during which time all the solids dissolved. After 20 min the reaction was complete, as determined by ${ }^{31} \mathrm{P}\left\{{ }^{1} \mathrm{H}\right\}$ NMR spectroscopy. The orange solution was filtered through Celite and concentrated. Addition of pentane led to precipitation of the product as an orange solid. The solid was filtered, washed with cold pentane, and dried under vacuum to give $126 \mathrm{mg}$ (58\% yield) of the product. ${ }^{1} \mathrm{H}$ NMR $\left(\mathrm{C}_{6} \mathrm{D}_{6}\right) \delta 0.80-2.80(\mathrm{~m}, 22 \mathrm{Cy} \mathrm{H}), 1.32(\mathrm{~d}, \mathrm{~J}=12.8 \mathrm{~Hz}, 9 \mathrm{H}), 1.61$ (t, J=7.2 Hz, 3H), $1.67(\mathrm{~d}, \mathrm{~J}=11.6 \mathrm{~Hz}, 9 \mathrm{H}), 2.19$ (s, 1H, 1/3 molecule of toluene), $3.10(\mathrm{~m}, 1 \mathrm{H})$, 3.98 (s, 5H), 4.01 (m, 1H), 4.04 (br s, 1H), 4.64 (br s, 1H), 7.01-7.06 (m, 2H), 7.10-7.15 (m, $\mathrm{C}_{6} \mathrm{D}_{6}$ and $1 / 3$ molecule of toluene), $7.35(\mathrm{br}, 1 \mathrm{H}), 7.84(\mathrm{br}, 2 \mathrm{H}) .{ }^{31} \mathrm{P}\left\{{ }^{1} \mathrm{H}\right\} \mathrm{NMR}\left(\mathrm{C}_{7} \mathrm{H}_{8},-20{ }^{\circ} \mathrm{C}\right) \delta$ $16.0(\mathrm{~d}, \mathrm{~J}=34.7 \mathrm{~Hz}), 72.0(\mathrm{~d}, \mathrm{~J}=34.7 \mathrm{~Hz})$. Anal. calc'd for $\mathrm{C}_{38} \mathrm{H}_{57} \mathrm{P}_{2} \mathrm{BrFePd} \bullet(1 / 3) \mathrm{C}_{7} \mathrm{H}_{8}: \mathrm{C}, 57.1$; H, 7.05. Found: C, 57.32; H, 7.19.

Synthesis of Pd(PPF-t-Bu)Cl . A suspension of $\mathrm{PdCl}_{2}(150 \mathrm{mg}, 0.85 \mathrm{mmol})$ in $15 \mathrm{~mL}$ of $\mathrm{CH}_{3} \mathrm{CN}$ was stirred at $80{ }^{\circ} \mathrm{C}$ until all solid dissolved. The warm solution was poured into a vial containing PPF- $t$ - $\mathrm{Bu}$ (490 mg, $0.903 \mathrm{mmol}$ ), and a red precipitate formed immediately. The suspension was allowed to cool to room temperature, and the red solid was isolated by filtration. The product was washed with pentane and dried under vacuum to produce $\mathrm{Pd}(\mathrm{PPF}-t-\mathrm{Bu}) \mathrm{Cl}_{2}(544$ $\mathrm{mg}, 85 \%$ yield) as a red, air-stable solid. ${ }^{1} \mathrm{H} \mathrm{NMR}\left(\mathrm{CDCl}_{3}\right) \delta 1.42(\mathrm{~d}, \mathrm{~J}=15.2 \mathrm{~Hz}, 9 \mathrm{H}), 1.59$ (d, $\mathrm{J}=13.6 \mathrm{~Hz}, 9 \mathrm{H}), 2.07(\mathrm{t}, \mathrm{J}=8.8 \mathrm{~Hz}, 3 \mathrm{H}), 3.17(\mathrm{~m}, 1 \mathrm{H}), 3.86(\mathrm{~s}, 1 \mathrm{H}), 3.95(\mathrm{~s}, 5 \mathrm{H}), 4.40(\mathrm{~s}, 1 \mathrm{H})$, 4.60 (s, 1H), 7.30-7.40 (m, 4H), 7.59 (br s, 4H), 8.47-8.52 (m, 2H). ${ }^{13} \mathrm{C}\left\{{ }^{1} \mathrm{H}\right\} \operatorname{NMR}\left(\mathrm{CDCl}_{3}\right) \delta$ 17.95 (d, J=5.1 Hz), 31.33 (d, 2.1 Hz), 31.61 (d, $2.9 \mathrm{~Hz}), 33.81$ (br t, J=9.8 Hz), 40.45 (d, $\mathrm{J}=11.0 \mathrm{~Hz}), 41.59$ (d, J=8.7 Hz), 69.09 (d, J=11.1 Hz), 69.65 (d, J=7.7 Hz), 70.50, 74.18 (d, J=5.6 $\mathrm{Hz}), 127.53$ (d, J=12.6 Hz), 128.35 (d, J=11.6 Hz), 129.85 (d, J=55.7 Hz), 130.46 (d, J=3.5 Hz), 131.71 (d, J=2.6 Hz), 132.19 (d, J=71.8 Hz), 134.02 (d, J=9.7 Hz), 135.46 (d, J=12.0 Hz). ${ }^{31} \mathrm{P}\left\{{ }^{1} \mathrm{H}\right\} \mathrm{NMR}\left(\mathrm{CD}_{2} \mathrm{Cl}_{2}\right) \delta 24.3(\mathrm{~s}), 112.2$ (s). Anal. calc'd for $\mathrm{C}_{32} \mathrm{H}_{40} \mathrm{P}_{2} \mathrm{Cl}_{2} \mathrm{FePd}: \mathrm{C}, 53.40 ; \mathrm{H}$, 5.61. Found: C, 53.19; H, 5.65. 
General Procedure for the Oxidative Addition of PhOTs to 1 and 2. Into a small vial was placed $10.0 \mathrm{mg}(0.0140 \mathrm{mmol})$ of $\mathrm{Pd}\left[\mathrm{P}(o-\mathrm{tol})_{3}\right]_{2}, 7.8 \mathrm{mg}(0.0140 \mathrm{~mol})$ of CyPF- $t$-Bu, and $5.4 \mathrm{mg}$ of $\mathrm{PMes}_{3}$ as internal standard. The solid materials were dissolved in $0.60 \mathrm{~mL} \mathrm{C}_{6} \mathrm{H}_{6}$, and the solution was transferred to an NMR tube. $\mathrm{A}^{31} \mathrm{P}\left\{{ }^{1} \mathrm{H}\right\}$ NMR spectrum was obtained. The solution was poured into a vial containing $17.3 \mathrm{mg}(0.0697 \mathrm{mmol})$ of PhOTs, and the resulting solution was stirred at room temperature. After $30 \mathrm{~min}$, a second ${ }^{31} \mathrm{P}\left\{{ }^{1} \mathrm{H}\right\}$ NMR spectrum was obtained, and this spectrum showed all starting material had been consumed and that compound $\mathbf{4}$ was produced in $92 \%$ yield.

\section{Measurement of Rate Constant for Oxidative Addition of Aryl Tosylate to Palladium(0):}

Representative Procedure. Into a small vial was placed $12.0 \mathrm{mg}(0.0170 \mathrm{mmol})$ of $\mathrm{Pd}[\mathrm{P}(\mathrm{o}-$ tolyl $\left.)_{3}\right]_{2}, 9.2 \mathrm{mg}(0.017 \mathrm{mmol})$ of PPF- $t$-Bu, $34.0 \mathrm{mg}(0.140 \mathrm{mmol})$ of PhOTs, $92.0 \mathrm{mg}(0.170$ $\mathrm{mmol})$ of $\mathrm{N}(\text { octyl })_{4} \mathrm{Br}, 26.0 \mathrm{mg}(0.0850 \mathrm{mmol})$ of $\mathrm{P}(o \text {-tolyl })_{3}$, and $\mathrm{PMes}_{3}$ (as internal standard). The solids were dissolved in $0.60 \mathrm{~mL}$ THF, and the solution was transferred to an NMR tube with a septum-lined, screw cap. The sample was heated at $55{ }^{\circ} \mathrm{C}$, and ${ }^{31} \mathrm{P}\left\{{ }^{1} \mathrm{H}\right\} \mathrm{NMR}$ spectra were obtained every 3 min for at least 5 half-lives by an automated program.

\section{Kinetic data:}

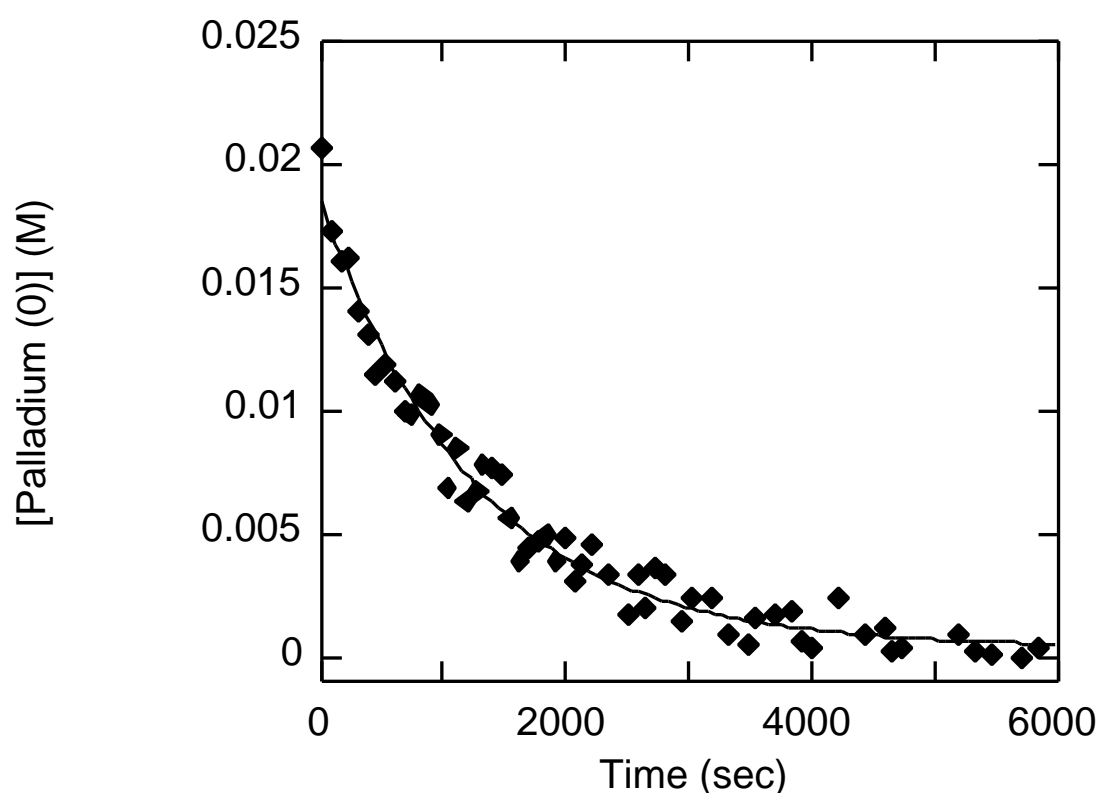

Figure $\mathrm{S} 1$. $[\mathrm{Pd}(0)]$ versus time for the oxidative addition of PhOTs to $\mathbf{1 .}$ 
Table S1. Dependence of $\mathrm{k}_{\mathrm{obs}}$ on $[\mathrm{Pd}]_{0}$.

\begin{tabular}{cc}
\hline$[\mathrm{Pd}]_{0}(\mathrm{M})$ & $\mathrm{k}_{\mathrm{obs}}\left(\mathrm{s}^{-1}\right) \times 10^{4}$ \\
\hline 0.0280 & 3.61 \\
0.111 & 3.49 \\
\hline
\end{tabular}

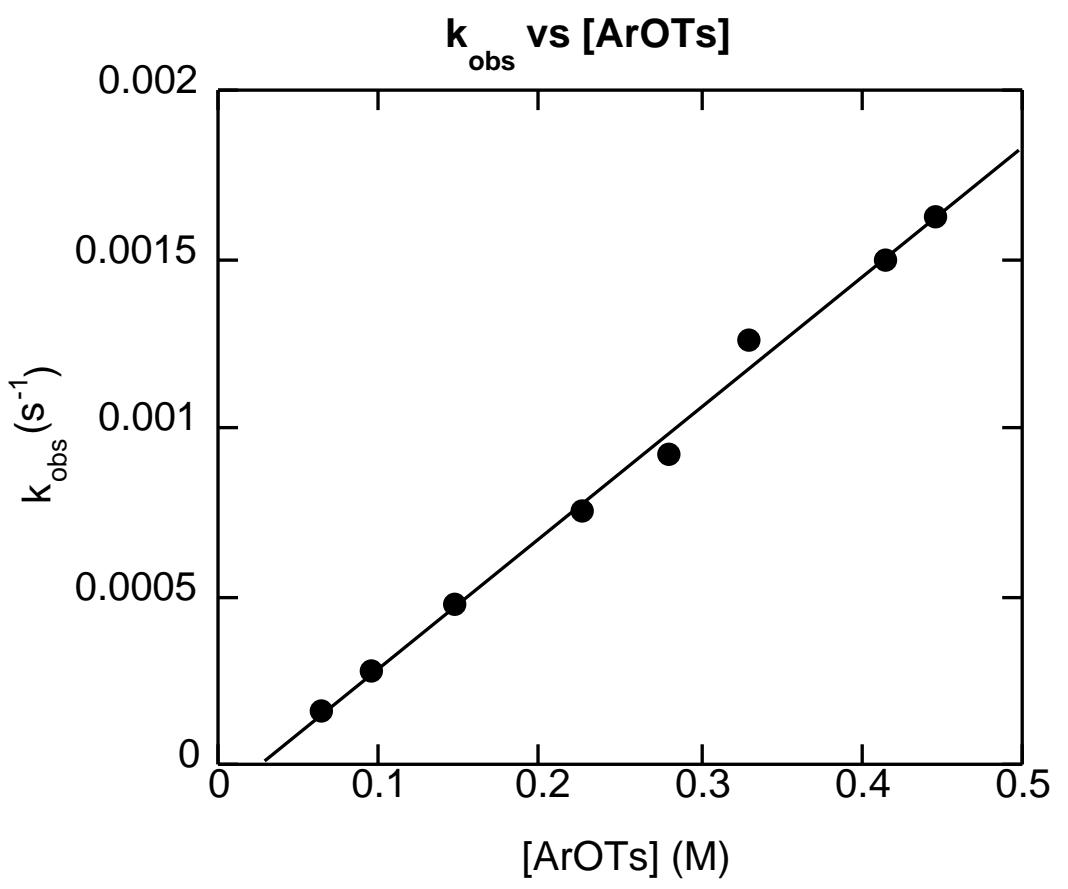

Figure S2. Dependence of $\mathrm{k}_{\mathrm{obs}}$ on [ArOTs].

Table S2. Dependence of $\mathrm{k}_{\mathrm{obs}}$ on [ArOTs].

\begin{tabular}{cc}
\hline$[$ ArOTs $]$ & $\mathrm{k}_{\text {obs }}\left(\mathrm{s}^{-1}\right) \times 10^{4}$ \\
\hline 0.0651 & 1.58 \\
0.0946 & 2.74 \\
0.148 & 4.78 \\
0.226 & 7.50 \\
0.280 & 9.17 \\
0.331 & 12.6 \\
0.414 & 15.0 \\
0.447 & 16.2 \\
\hline
\end{tabular}




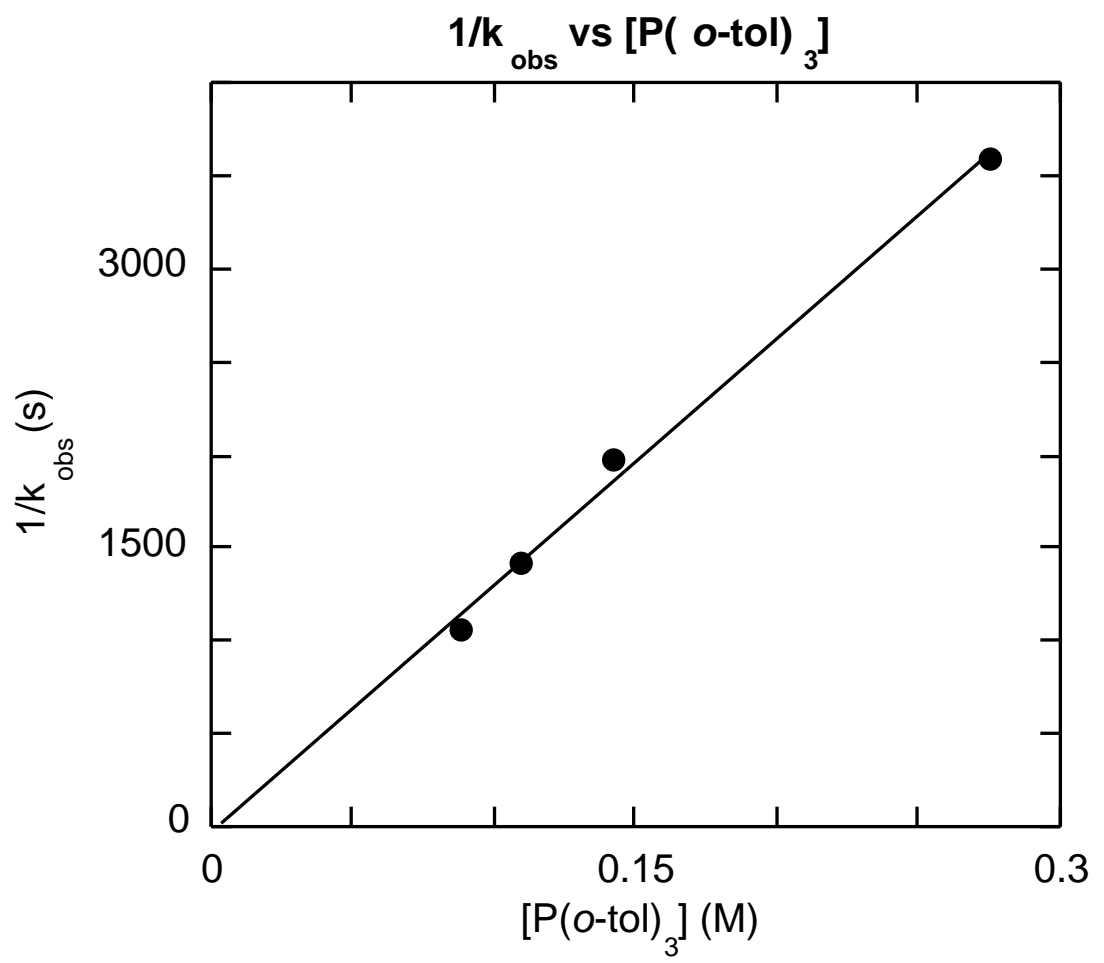

Figure S3. Dependence of $\mathrm{k}_{\mathrm{obs}}$ on $\left[\mathrm{P}(o-\text { tol })_{3}\right]$.

Table S3. Dependence of $\mathrm{k}_{\mathrm{obs}}$ on $\left[\mathrm{P}(o-\mathrm{tol})_{3}\right]$.

\begin{tabular}{ccc}
\hline$\left[\mathrm{P}(o-\text { tol })_{3}\right](\mathrm{M})$ & $\mathrm{k}_{\mathrm{obs}}\left(\mathrm{s}^{-1}\right) \times 10^{4}$ & $1 / \mathrm{k}_{\mathrm{obs}}(\mathrm{s}) \times 10^{-3}$ \\
\hline 0.0882 & 9.38 & 1.07 \\
0.110 & 7.03 & 1.42 \\
0.142 & 5.06 & 1.98 \\
0.275 & 2.79 & 3.58 \\
\hline
\end{tabular}




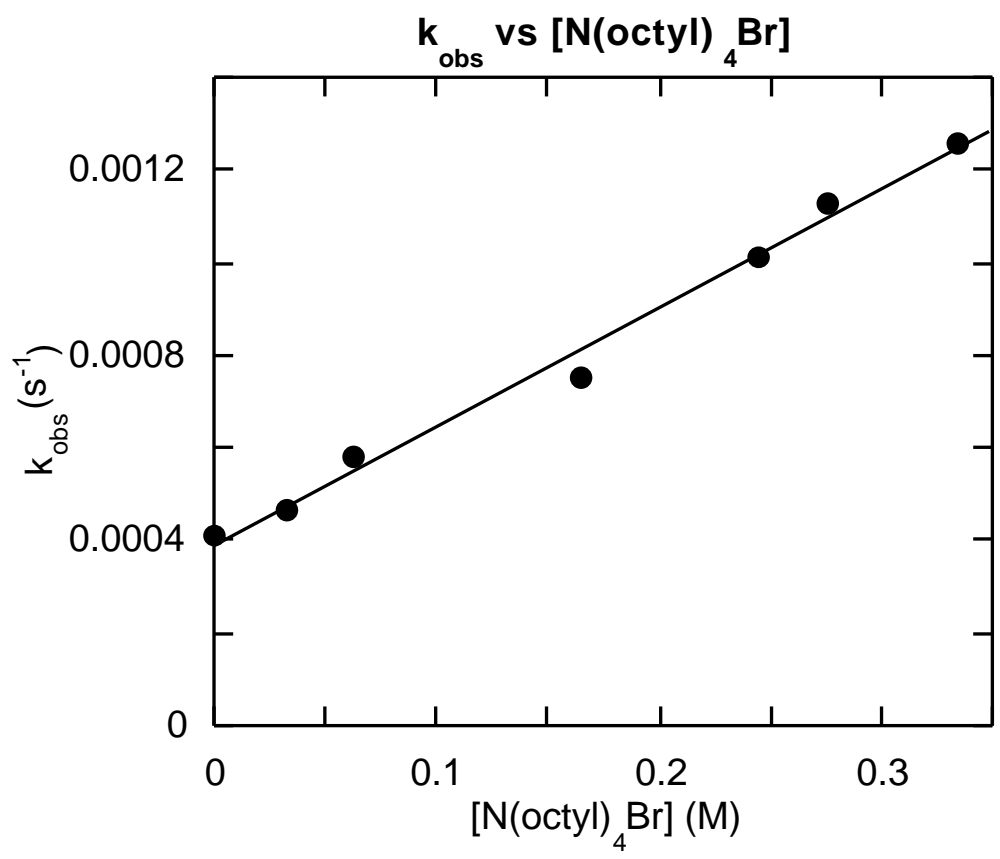

Figure S4. Dependence of $\mathrm{k}_{\mathrm{obs}}$ on $\left[\mathrm{N}(\text { octyl })_{4} \mathrm{Br}\right]$.

Table S4. Dependence of $\mathrm{k}_{\mathrm{obs}}$ on [N(octyl) $)_{4} \mathrm{Br}$ ].

\begin{tabular}{cc}
\hline$\left[\mathrm{N}\left(\right.\right.$ octyl $\left._{4} \mathrm{Br}\right](\mathrm{M})$ & $\mathrm{k}_{\mathrm{obs}}\left(\mathrm{s}^{-1}\right) \times 10^{4}$ \\
\hline 0 & 4.11 \\
0.0329 & 4.63 \\
0.0628 & 5.83 \\
0.165 & 7.50 \\
0.244 & 10.1 \\
0.277 & 11.3 \\
0.335 & 12.6 \\
\hline
\end{tabular}




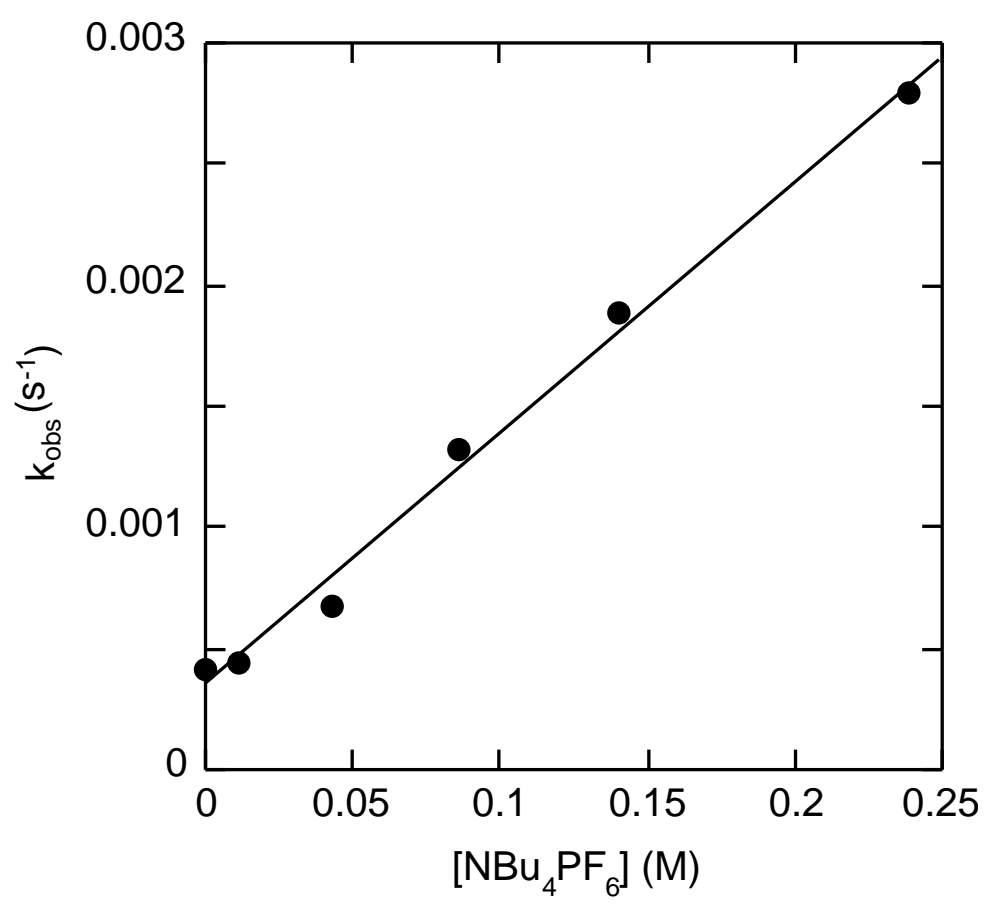

Figure S5. Dependence of $\mathrm{k}_{\mathrm{obs}}$ on $\left[\mathrm{NBu}_{4} \mathrm{PF}_{6}\right]$.

Table S5. Dependence of $\mathrm{k}_{\mathrm{obs}}$ on $\left[\mathrm{NBu}_{4} \mathrm{PF}_{6}\right]$.

\begin{tabular}{cc}
\hline$\left[\mathrm{NBu}_{4} \mathrm{PF}_{6}\right](\mathrm{M})$ & $\mathrm{k}_{\mathrm{obs}}\left(\mathrm{s}^{-1}\right) \times 10^{4}$ \\
\hline 0 & 4.11 \\
0.0116 & 4.41 \\
0.0426 & 6.81 \\
0.0865 & 13.2 \\
0.140 & 18.8 \\
0.239 & 27.9 \\
\hline
\end{tabular}


General Procedure for the Reaction of Aryl Tosylates with Arylmagnesium Bromides. The reaction conditions and average yields for each reaction are shown in Table 1. A typical procedure is given for the first entry in Table 1.

4-Methoxy-4'-methylbiphenyl. ${ }^{4}$ Into a small vial was placed $5.8 \mathrm{mg}(0.010 \mathrm{mmol})$ of $\mathrm{Pd}(\mathrm{dba})_{2}$, $5.4 \mathrm{mg}(0.010 \mathrm{mmol})$ of PPF- $t$ - Bu, and $278.3 \mathrm{mg}(1.0 \mathrm{mmol})$ of $4-\mathrm{MeOC}_{6} \mathrm{H}_{4} \mathrm{OTs}$. The solids were dissolved in $1.5 \mathrm{~mL}$ of toluene. To the vial, $2.00 \mathrm{~mL}(2.0 \mathrm{mmol})$ of a $1.0 \mathrm{M}$ solution of $p$ tolyl $\mathrm{MgBr}$ in ether were added by syringe. The vial was sealed with a cap containing a PTFE septum, and the mixture was stirred at room temperature until the aryl tosylate was consumed, as determined by GC. The mixture was hydrolyzed with $1.0 \mathrm{M} \mathrm{HCl}$, and the product was extracted with diethyl ether. The organic extracts were washed with saturated sodium bicarbonate and brine and then dried over $\mathrm{MgSO}_{4}$. After filtration and evaporation of the solvent, the residue was purified by chromatography on silica gel (hexane/ethyl acetate $=99 / 1)$ to give $174.5 \mathrm{mg}(88 \%$ yield) of 4-methoxy-4'-methylbiphenyl. ${ }^{1} \mathrm{H}$ NMR $\left(\mathrm{C}_{6} \mathrm{D}_{6}\right) \delta 2.17(\mathrm{~s}, 3 \mathrm{H}), 3.33(\mathrm{~s}, 3 \mathrm{H}), 6.85$ (m, $2 \mathrm{H}), 7.07(\mathrm{~d}, \mathrm{~J}=4.0 \mathrm{~Hz}, 2 \mathrm{H}), 7.44(\mathrm{~m}, 4 \mathrm{H}) .{ }^{13} \mathrm{C} \mathrm{NMR}\left(\mathrm{CDCl}_{3}\right) \delta 21.01,55.26,114.13,126.54$, $127.90,129.40,133.70,136.28,137.93,158.91$.

4-Methoxy-2'-methylbiphenyl. ${ }^{5}$ 4- $\mathrm{MeOC}_{6} \mathrm{H}_{4} \mathrm{OTs}(282 \mathrm{mg}, 1.01 \mathrm{mmol}), o$-tolylMgBr $(1.0 \mathrm{~mL}$ of a $2 \mathrm{M}$ solution in ether, $2.00 \mathrm{mmol}), \mathrm{Pd}(\mathrm{dba})_{2}(5.8 \mathrm{mg}, 0.010 \mathrm{mmol})$, and PPF- $t$-Bu $(5.4 \mathrm{mg}$, $0.010 \mathrm{mmol})$ were used. The crude product was purified by chromatography on silica gel (hexane/ethyl acetate $=99 / 1)$ to give $147 \mathrm{mg}(73 \%$ yield $)$ of 4-methoxy-2'-methylbiphenyl. ${ }^{1} \mathrm{H}$ NMR $\left(\mathrm{CD}_{2} \mathrm{Cl}_{2}\right) \delta 2.26(\mathrm{~s}, 3 \mathrm{H}), 3.84(\mathrm{~s}, 3 \mathrm{H}), 6.94-6.96(\mathrm{~m}, 2 \mathrm{H}), 7.20-7.26(\mathrm{~m}, 6 \mathrm{H}) .{ }^{13} \mathrm{C} \mathrm{NMR}$ $\left(\mathrm{CD}_{2} \mathrm{Cl}_{2}\right) \delta 20.70,55.61,113.85,126.12,127.33,130.19,130.63,130.66,134.70,135.86$, $141.97,159.05$.

4-Methyl-4'-trifluoromethylbiphenyl. 6 4- $\mathrm{CF}_{3} \mathrm{C}_{6} \mathrm{H}_{4} \mathrm{OTs}$ (316 mg, $1.00 \mathrm{mmol}$ ), p-tolylMgBr $(2.0 \mathrm{~mL}$ of a $1.0 \mathrm{M}$ solution in ether, $2.00 \mathrm{mmol}), \mathrm{Pd}(\mathrm{dba})_{2}(5.8 \mathrm{mg}, 0.010 \mathrm{mmol})$, and PPF- $t$-Bu $(5.4 \mathrm{mg}, 0.010 \mathrm{mmol})$ were used. The crude product was purified by chromatography on silica gel (hexane/ethyl acetate $=99 / 1)$ to give $154 \mathrm{mg}$ (65\% yield) of 4-methyl-4'trifluoromethylbiphenyl. ${ }^{1} \mathrm{H}$ NMR $\left(\mathrm{CD}_{2} \mathrm{Cl}_{2}\right) \delta 2.41(\mathrm{~s}, 3 \mathrm{H}), 7.30(\mathrm{dd}, \mathrm{J}=8.5 \mathrm{~Hz}, 1.0 \mathrm{~Hz}, 2 \mathrm{H}), 7.53$ (dd, J=6.3 Hz, 1.5 Hz, 2H), 7.68-7.73 (m, 4H). ${ }^{13} \mathrm{C} \mathrm{NMR}\left(\mathrm{CD}_{2} \mathrm{Cl}_{2}\right) \delta 21.63,124.89$ (q, J=272 Hz, $\mathrm{CF}_{3}$ ), 125.98 (q, J=3.8 Hz), 127.36, 127.50, 129.08 (q, J=32.0 Hz), 130.05, 136.98, 138.73, 145.03.

1-p-Tolylnaphthalene. ${ }^{7}$ Toluene-4-sulfonic acid-[1]naphthyl ester (298 mg, $\left.1.00 \mathrm{mmol}\right)$, tolylMgBr (2.0 mL of a $1.0 \mathrm{M}$ solution in ether, $2.00 \mathrm{mmol}), \mathrm{Pd}(\mathrm{dba})_{2}(5.8 \mathrm{mg}, 0.010 \mathrm{mmol})$, and PPF-t-Bu (5.4 mg, $0.010 \mathrm{mmol})$ were used. The crude product was purified by chromatography 
on silica gel (hexanes). The residue was dissolved in ether, layered with pentane, and recrystallized at $-35{ }^{\circ} \mathrm{C}$ to give $171 \mathrm{mg}$ (78\% yield) of 1-p-tolylnaphthalene. ${ }^{1} \mathrm{H} \mathrm{NMR}\left(\mathrm{CD}_{2} \mathrm{Cl}_{2}\right)$ $\delta 2.46(\mathrm{~s}, 3 \mathrm{H}), 7.32-7.55(\mathrm{~m}, 8 \mathrm{H}), 7.86(\mathrm{~d}, 6.5 \mathrm{~Hz}, 1 \mathrm{H}), 7.92(\mathrm{~d}, 4.1 \mathrm{~Hz}, 2 \mathrm{H}) .{ }^{13} \mathrm{C} \mathrm{NMR}\left(\mathrm{CD}_{2} \mathrm{Cl}_{2}\right)$ $\delta 21.55,126.00,126.30,126.53,126.55,127.19,127.47,127.96,128.81,129.55,130.49,132.28$, $134.48,137.65,140.40$.

4-Fluoro-4'-methylbiphenyl. 8 4-MeC6 $\mathrm{H}_{4} \mathrm{OTs}$ (262 mg, $\left.1.00 \mathrm{mmol}\right)$, p-FC6H4MgBr (1.0 mL of a $2.0 \mathrm{M}$ solution in tetrahydrofuran, $2.00 \mathrm{mmol}), \mathrm{Pd}(\mathrm{dba}) 2$ and PPF-t-Bu $(100 \mu \mathrm{L}$ of a $0.010 \mathrm{M}$ solution of precatalyst and ligand, $0.0010 \mathrm{mmol}$ ) were used. The residue was dissolved in ether, layered with pentane, and recrystallized at $-35^{\circ} \mathrm{C}$ to give $125 \mathrm{mg}$ (67\% yield) of 4-fluoro-4'methylbiphenyl. ${ }^{1} \mathrm{H}$ NMR $\left(\mathrm{CD}_{2} \mathrm{Cl}_{2}\right) \delta 2.38(\mathrm{~s}, 3 \mathrm{H}), 7.10-7.15(\mathrm{~m}, 2 \mathrm{H}), 7.25(\mathrm{~d}, \mathrm{~J}=4.0 \mathrm{~Hz}, 2 \mathrm{H})$, $7.45(\mathrm{~d}, \mathrm{~J}=3.2 \mathrm{~Hz}, 2 \mathrm{H}), 7.51-7.57(\mathrm{~m}, 2 \mathrm{H}) .{ }^{13} \mathrm{C} \mathrm{NMR}\left(\mathrm{CD}_{2} \mathrm{Cl}_{2}\right) \delta 21.35,115.97$ (d, J=20.9 Hz), 127.29, 128.99 (d, J=7.5 Hz), 130.08, 137.74, 137.78, 137.86 (d, J=4.4 Hz), 162.87 (d, J=245.3 $\mathrm{Hz})$.

2-Methoxy-4'-methylbiphenyl. 9 2-MeOC ${ }_{6} \mathrm{H}_{4} \mathrm{OTs}(278 \mathrm{mg}, 1.00 \mathrm{mmol})$, $p$-tolylMgBr $(2.0 \mathrm{~mL}$, $1.0 \mathrm{M}$ solution in ether, $2.00 \mathrm{mmol}), \mathrm{Pd}(\mathrm{dba})_{2}(5.8 \mathrm{mg}, 0.010 \mathrm{mmol})$, and PPF- $t$-Bu $(5.4 \mathrm{mg}$, $0.010 \mathrm{mmol}$ ) were used. The crude product was purified by chromatography on silica gel (hexane/ethyl acetate $=99 / 1)$ to give $90 \mathrm{mg}\left(45 \%\right.$ yield) of 2-methoxy-4'-methylbiphenyl. ${ }^{1} \mathrm{H}$ $\operatorname{NMR}\left(\mathrm{CD}_{2} \mathrm{Cl}_{2}\right) \delta 2.38(\mathrm{~s}, 3 \mathrm{H}), 3.80(\mathrm{~s}, 3 \mathrm{H}), 6.98-7.02(\mathrm{~m}, 2 \mathrm{H}), 7.21(\mathrm{~d}, \mathrm{~J}=4.0 \mathrm{~Hz}, 2 \mathrm{H}), 7.27-7.32$ $(\mathrm{m}, 2 \mathrm{H}), 7.38-7.40(\mathrm{~m}, 2 \mathrm{H}) .{ }^{13} \mathrm{C} \mathrm{NMR}\left(\mathrm{CD}_{2} \mathrm{Cl}_{2}\right) \delta 20.22,55.85,111.22,121.00,129.21,130.12$, $130.58,131.36,131.46,137.49,139.47,157.23$.

2-Methoxy-2'-methylbiphenyl. 5 2-MeOC ${ }_{6} \mathrm{H}_{4} \mathrm{OTs}(278 \mathrm{mg}, 1.00 \mathrm{mmol}), o$-tolylMgBr $(1.0 \mathrm{~mL}$, 2.0 M solution in ether, $2.00 \mathrm{mmol}), \mathrm{Pd}(\mathrm{dba})_{2}$ and PPF- $t$-Bu $(100 \mu \mathrm{L}$ of a $0.010 \mathrm{M}$ solution of precatalyst and ligand, $0.0010 \mathrm{mmol}$ ) were used. The crude product was purified by column chromatography on silica gel (hexanes to elute 2,2'-dimethylbiphenyl, then 95/5 hexane/ethyl acetate to elute 2-methoxy-2'-methylbiphenyl) to give $112 \mathrm{mg}$ (56\% yield) of 2-methoxy-2'methylbiphenyl. ${ }^{1} \mathrm{H}$ NMR $\left(\mathrm{CD}_{2} \mathrm{Cl}_{2}\right) \delta 2.11(\mathrm{~s}, 3 \mathrm{H}), 3.76(\mathrm{~s}, 3 \mathrm{H}), 6.98-7.03(\mathrm{~m}, 2 \mathrm{H}), 7.11-7.15$ $(\mathrm{m}, 2 \mathrm{H}), 7.19-7.25(\mathrm{~m}, 3 \mathrm{H}), 7.33-7.39(\mathrm{~m}, 1 \mathrm{H}) .{ }^{13} \mathrm{C} \mathrm{NMR}\left(\mathrm{CD}_{2} \mathrm{Cl}_{2}\right) \delta 20.02,55.65,110.01$, $120.80,125.78,127.59,129.00,129.92,130.37,131.16,131.25,137.29,139.27,157.03$.

2-Methyl-2',4',6'-trimethylbiphenyl. ${ }^{8}$ MesOTs (290 mg, $\left.1.00 \mathrm{mmol}\right)$, -tolylMgBr $(2.0 \mathrm{~mL}$ of a $1.0 \mathrm{M}$ solution, $2.00 \mathrm{mmol}), \mathrm{Pd}(\mathrm{dba})_{2}(5.8 \mathrm{mg}, 0.010 \mathrm{mmol})$, and PPF-t-Bu (5.4 mg, 0.010 $\mathrm{mmol}$ ) were used. The crude product was purified by column chromatography on silica gel (hexanes), then recrystallization from pentane at $-35{ }^{\circ} \mathrm{C}$ provided $84 \mathrm{mg}$ (40\% yield) of 2methyl-2', 4', 6'-trimethylbiphenyl. ${ }^{1} \mathrm{H}$ NMR $\left(\mathrm{CDCl}_{3}\right) \delta 1.99(\mathrm{~s}, 6 \mathrm{H}), 2.30(\mathrm{~s}, 3 \mathrm{H}), 2.38(\mathrm{~s}, 3 \mathrm{H})$, 
$6.91(\mathrm{~s}, 2 \mathrm{H}), 7.00(\mathrm{~d}, \mathrm{~J}=4.0 \mathrm{~Hz}, 2 \mathrm{H}), 7.20(\mathrm{~d}, \mathrm{~J}=4.0 \mathrm{~Hz}, 2 \mathrm{H}) .{ }^{13} \mathrm{C} \mathrm{NMR}\left(\mathrm{CD}_{2} \mathrm{Cl}_{2}\right) \delta 20.88,21.09$, 21.30, 128.28, 129.36, 129.50 (2 overlapping C), 129.59, 129.78, 129.97, 136.22, 136.40, 136.64 .

$N$-octylaniline. ${ }^{10}$ Into a $20 \mathrm{~mL}$ vial was placed $5.8 \mathrm{mg}(0.010 \mathrm{mmol}) \mathrm{PdCl}_{2}(\mathrm{PPF}-t-\mathrm{Bu}), 202 \mathrm{mg}$ $(0.81 \mathrm{mmol})$ of PhOTs, and $200 \mathrm{mg}(2.08 \mathrm{mmol})$ of $\mathrm{NaO}(t-\mathrm{Bu})$. The solids were dissolved in 15 $\mathrm{mL}$ of toluene, and $160 \mu \mathrm{L}(0.97 \mathrm{mmol})$ of octylamine were added by syringe into the vial. The mixture was stirred at room temperature until the aryl tosylate was consumed, as determined by GC, and then diluted with EtOAc. After filtration and evaporation of the solvent, the residue was purified by chromatography on silica gel (hexane/ethyl acetate $=95 / 5)$ to give $128 \mathrm{mg}(76 \%$ yield) of $N$-octylaniline. ${ }^{1} \mathrm{H}$ NMR $\left(\mathrm{CD}_{2} \mathrm{Cl}_{2}\right) \delta 0.87-0.91(\mathrm{~m}, 3 \mathrm{H}), 1.29-1.41(\mathrm{~m}, 10 \mathrm{H}), 1.59$ (quintet, J=7.6 Hz, 2H), 3.08 (t, J=7.2 Hz, 2H), 3.66 (br s, 1H), 6.57-6.65 (m, 3H), 7.11-7.15 (m, $2 \mathrm{H}) .{ }^{13} \mathrm{C} \mathrm{NMR}\left(\mathrm{CD}_{2} \mathrm{Cl}_{2}\right) \delta 14.54,23.34,27.84,29.95,30.10,30.22,32.52,44.57,113.13$, $117.38,129.72,149.42$. 


\section{References:}

1)Paul, F.; Patt, J.; Hartwig, J. F. Organometallics 1995, 14, 3030.

2)Komiya, S. ; John Wiley \& Sons: New York, 1997, pp 290.

3)Burckhardt, U.; Gramlich, V.; Hofmann, P.; Nesper, R.; Pregosin, P. S.; Salzmann, R.; Togni, A. Organometallics 1996, 15, 3496.

4)Farina, V.; Krishnan, B.; Marshall, D. R.; Roth, G. P. J. Org. Chem. 1993, 58, 5434.

5)Lipshutz, B. H.; Siegmann, K.; Garcia, E.; Kayser, F. J. Am. Chem. Soc. 1993, 115, 9276.

6)Gouda, K.; Hagiwara, E.; Hatanaka, Y.; Hiyama, T. J. Org. Chem. 1996, 7232.

7)Leznoff, C. C.; Hayward, R. J. Can. J. Chem. 1970, 48, 1842.

8)Bell, H. C.; Kalman, J. R.; May, G. L.; Pinhey, J. T.; Sternhell, S. Aust. J. Chem. 1979, 32, 1531.

9)Wolfe, J. P.; Singer, R. A.; Yang, B. H.; Buchwald, S. L. J. Am. Chem. Soc. 1999, 121, 9550.

10)Cho, C. S.; Kim, J. S.; Kim, H. S.; Kim, T. J.; Shim, S. C. Synth. Commun. 2001, 31, 3791. 


\section{Crystallographic data for 5.}

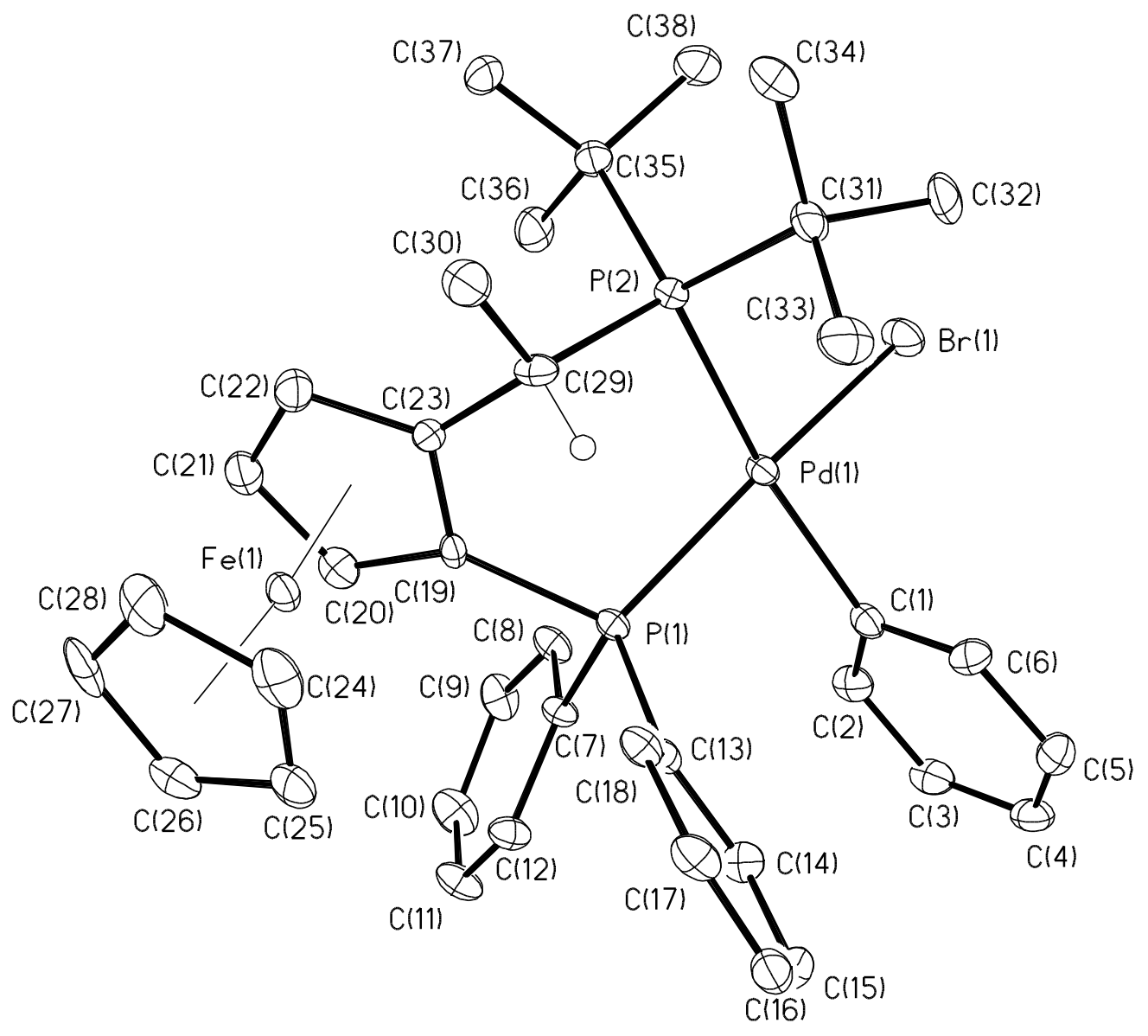

Figure S6. X-ray crystal structure of $\mathbf{5}$.

\section{Data Collection}

An orange needle crystal of $\mathrm{C}_{42} \mathrm{H}_{53} \mathrm{OP}_{2} \mathrm{BrFePd}$ having approximate dimensions of $0.10 \times 0.15$ x $0.29 \mathrm{~mm}$ was mounted on a glass fiber. All measurements were made on a Nonius KappaCCD diffractometer with graphite monochromated Mo-K $\alpha$ radiation.

Cell constants and an orientation matrix for data collection, obtained from a least-squares refinement using ten $\left(1^{\circ}\right.$ in $\omega, 10$ s exposure, de-zingered) data frames, corresponded to a primitive monoclinic cell with dimensions: $a=9.4928(2) \AA, b=15.1888(5) \AA, c=13.5344(4)$ 
$\AA, \beta=91.7135^{\circ}$ and $\mathrm{V}=1950.57(8) \AA^{3}$. For $\mathrm{Z}=2$ and F.W. $=877.98$, the calculated density is $1.50 \mathrm{~g} / \mathrm{cm}^{3}$. Based on the systematic absences of: $0 \mathrm{k} 0: \mathrm{k}=2 \mathrm{n}+1$; packing considerations, a statistical analysis of intensity distribution, and the successful solution and refinement of the structure, the space group was determined to be: $\mathrm{P} 2{ }_{1}(\# 4)$. In this acentric space group, the enantiomer was selected on the basis of refinement of the flack parameter and on the leastsquares refinement $\mathrm{R}$ values (the $\mathrm{R}$ values of the opposite hand were $\mathrm{r}$, rw: $6.37,7.46 \%$ ).

The data were collected at a temperature of $-90 \pm 1^{\circ} \mathrm{C}$ to a maximum $2 \theta$ value of $61.2^{\circ}$. One phi scan consisting of 140 data frames and two omega scans consisting of 44 and 32 data frames, respectively, were collected with a scan width of $1^{\circ}$ and a detector-to-crystal distance, Dx, of $35 \mathrm{~mm}$. Each frame was exposed twice (for the purpose of de-zingering) for 20s. The data frames were processed and scaled using the DENZO software package. (Z. Otwinowski and W. Minor, "Processing of X-Ray Diffraction Data Collected in Oscillation Mode," Methods in Enzymology, vol. 276: Macromolecular Crystallography, part A, 307-326, 1997, C.W. Carter, Jr. \& R.M. Sweet, Eds., Academic Press).

\section{Data Reduction}

Of the 13797 reflections which were collected, 5823 were unique ( $R_{\text {int }}=0.061$ ); equivalent reflections were merged. No decay correction was applied. The linear absorption coefficient, $\mu$, for Mo-K $\beta$ radiation is $19.7 \mathrm{~cm}^{-1}$ and a SORTAV absorption correction was applied (MaXus: Mackay, S., Edwards, C., Henderson, A., Gilmore, C., Stewart, N., Shankland, K. and Donald, A. (1998) Chemistry Department, The University of Glasgow, Scotland.) The data were corrected for Lorentz and polarization effects. A correction for secondary extinction was applied (coefficient $=2.82590 \mathrm{e}-07)$.

\section{$\underline{\text { Structure Solution and Refinement }}$}

The structure was solved by direct methods and expanded using Fourier techniques ${ }^{2}$. The non-hydrogen atoms were refined anisotropically. Hydrogen atoms were included but not

refined. The final cycle of full-matrix least-squares refinement ${ }^{3}$ was based on 4363 observed reflections (I > 3.00F(I)) and 432 variable parameters and converged (largest parameter shift was 0.00 times its esd) with unweighted and weighted agreement factors of: 


$$
\begin{gathered}
\mathrm{R}=\Sigma\|\mathrm{Fo}|-| \mathrm{Fc}\| / \Sigma|\mathrm{Fo}|=0.042 \\
\mathrm{R}_{\mathrm{W}}=\left[\left(\Sigma \mathrm{w}(|\mathrm{Fo}|-|\mathrm{Fc}|)^{2} / \Sigma \mathrm{w} \mathrm{Fo}^{2}\right)\right]^{1 / 2}=0.041
\end{gathered}
$$

The standard deviation of an observation of unit weight ${ }^{4}$ was 1.37 . The weighting scheme was based on counting statistics and included a factor $(p=0.020)$ to downweight the intense reflections. Plots of $\Sigma \mathrm{w}(|\mathrm{Fo}|-|\mathrm{Fc}|)^{2}$ versus $|\mathrm{Fo}|$, reflection order in data collection, sin $\theta / \lambda$ and various classes of indices showed no unusual trends. The maximum and minimum peaks on the final difference Fourier map corresponded to 0.70 and $-1.34 \mathrm{e}^{-} / \AA^{3}$, respectively.

Neutral atom scattering factors were taken from Cromer and Waber 5 . Anomalous dispersion effects were included in Fcalc 6 ; the values for $\Delta \mathrm{f}^{\prime}$ and $\Delta \mathrm{f}^{\prime \prime}$ were those of Creagh and McAuley 7 . The values for the mass attenuation coefficients are those of Creagh and Hubbel ${ }^{8}$. All calculations were performed using the teXsan ${ }^{9}$ crystallographic software package of Molecular Structure Corporation.

\section{References}

(1) ORIENT: Beurskens, P.T., Admiraal, G., Beurskens, G., Bosman, W.P., Garcia-Granda, S., Gould, R.O., Smits, J.M.M. and Smykalla, C. (1992). The DIRDIF program system, Technical Report of the Crystallography Laboratory, University of Nijmegen, The Netherlands.

(2) DIRDIF94: Beurskens, P.T., Admiraal, G., Beurskens, G., Bosman, W.P., de Gelder, R., Israel, R. and Smits, J.M.M.(1994). The DIRDIF-94 program system, Technical Report of the Crystallography Laboratory, University of Nijmegen, The Netherlands.

(3) Least-Squares:

$$
\begin{aligned}
& \text { Function minimized } \Sigma w\left(\left|\mathrm{~F}_{\mathrm{o}}\right|-\left|\mathrm{F}_{\mathrm{c}}\right|\right)^{2} \\
& \qquad \begin{array}{l}
\text { where } w=4 \mathrm{~F}_{\mathrm{o}}^{2} / 2\left(\mathrm{~F}_{\mathrm{o}}{ }^{2}\right) \\
\text { and } \sigma^{2}\left(\mathrm{~F}_{\mathrm{o}}^{2}\right)=\left[\mathrm{S}^{2}\left(\mathrm{C}+\mathrm{R}^{2} \mathrm{~B}\right)+\left(p \mathrm{~F}_{\mathrm{o}}^{2}\right)^{2}\right] / \mathrm{Lp}^{2} \\
\mathrm{~S}=\text { Scan rate } \\
\mathrm{C}=\text { Total integrated peak count } \\
\mathrm{R}=\text { Ratio of scan time to background counting time }
\end{array}
\end{aligned}
$$




$$
\begin{aligned}
& \mathrm{B}=\text { Total background count } \\
& \mathrm{Lp}=\text { Lorentz-polarization factor } \\
& p=\mathrm{p} \text {-factor }
\end{aligned}
$$

(4) Standard deviation of an observation of unit weight:

$$
\begin{aligned}
& {\left[\Sigma w\left(\left|\mathrm{~F}_{\mathrm{O}}\right|-\left|\mathrm{F}_{\mathrm{c}}\right|\right)^{2} /\left(\mathrm{N}_{\mathrm{O}}-\mathrm{N}_{\mathrm{v}}\right)\right]^{1 / 2}} \\
& \text { where } \mathrm{N}_{\mathrm{O}}=\text { number of observations } \\
& \quad \mathrm{N}_{\mathrm{v}}=\text { number of variables }
\end{aligned}
$$

(5) Cromer, D. T. \& Waber, J. T.; "International Tables for X-ray Crystallography", Vol. IV, The Kynoch Press, Birmingham, England, Table 2.2 A (1974).

(6) Ibers, J. A. \& Hamilton, W. C.; Acta Crystallogr., 17, 781 (1964).

(7) Creagh, D. C. \& McAuley, W.J .; "International Tables for Crystallography", Vol C, (A.J.C. Wilson, ed.), Kluwer Academic Publishers, Boston, Table 4.2.6.8, pages 219-222 (1992).

(8) Creagh, D. C. \& Hubbell, J.H..; "International Tables for Crystallography", Vol C, (A.J.C. Wilson, ed.), Kluwer Academic Publishers, Boston, Table 4.2.4.3, pages 200-206 (1992).

(9) teXsan: Crystal Structure Analysis Package, Molecular Structure Corporation (1985 \& 1992) 


\section{EXPERIMENTAL DETAILS FOR 5}

A. Crystal Data

Empirical Formula

Formula Weight

Crystal Color, Habit

Crystal Dimensions

Crystal System

Lattice Type

Lattice Parameters

\section{$\mathrm{C}_{42} \mathrm{H}_{53} \mathrm{OP}_{2} \mathrm{BrFePd}$}

877.98

orange, needle

$0.10 \times 0.15 \times 0.29 \mathrm{~mm}$

monoclinic

Primitive

$$
\begin{aligned}
& a=9.4928(2) \AA \\
& b=15.1888(5) \AA \\
& c=13.5344(4) \AA \\
& \beta=91.7135^{\circ} \\
& V=1950.57(8) \AA^{3}
\end{aligned}
$$

Space Group

P2 1 (\#4)

$\mathrm{Z}$ value

2

Dcalc

$1.495 \mathrm{~g} / \mathrm{cm}^{3}$

$\mathrm{F}_{000}$

$\mu\left(\mathrm{MoK}^{\prime \prime}\right)$
900.00

$19.74 \mathrm{~cm}^{-1}$ 
B. Intensity Measurements

Diffractometer

Radiation

Take-off Angle

Crystal to Detector Distance

Temperature

Scan Rate

Scan Width

$2 \theta_{\max }$

No. of Reflections Measured

Corrections
Nonius KappaCCD

$\operatorname{MoK} \alpha(\lambda=0.71069 \AA)$

graphite monochromated

$2.8^{\circ}$

$35 \mathrm{~mm}$

$-90.0^{\circ} \mathrm{C}$

20s/frame

$10 /$ frame

$61.2^{0}$

Total: 13797

Unique: $5823\left(\mathrm{R}_{\text {int }}=0.061\right)$

Lorentz-polarization

Absorption (SORTAV)

Secondary Extinction

(coefficient: $2.82590 \mathrm{e}-07$ ) 


\section{Structure Solution and Refinement}

Structure Solution

Refinement

Function Minimized

Least Squares Weights

p-factor

Anomalous Dispersion

No. Observations (I>3.00F(I))

No. Variables

Reflection/Parameter Ratio

Residuals: R; Rw

Goodness of Fit Indicator

Max Shift/Error in Final Cycle

Maximum peak in Final Diff. Map

Minimum peak in Final Diff. Map
Direct Methods

Full-matrix least-squares

$\Sigma \mathrm{w}(|\mathrm{Fo}|-|\mathrm{Fc}|)^{2}$

$1 / \sigma^{2}(\mathrm{Fo})$

0.0200

All non-hydrogen atoms

4363

432

10.10

$0.042 ; 0.041$

1.37

0.00

$0.70 \mathrm{e}^{-/} \AA^{3}$

$-1.34 \mathrm{e}-/ \AA$ 
Table S6. Atomic coordinates and $\mathrm{B}_{\mathrm{iso}} / \mathrm{B}_{\mathrm{eq}}$ for $\mathbf{5}$.

$\begin{array}{lllll}\text { atom } & \mathrm{x} & \mathrm{y} & \mathrm{z} & \mathrm{B} \text { eq } \\ & & & & \\ \mathrm{Pd}(1) & -0.70967(4) & -0.3340 & -0.66182(3) & 1.560(8) \\ \mathrm{Br}(1) & -0.47271(6) & -0.34817(6) & -0.57262(4) & 2.79(1) \\ \mathrm{Fe}(1) & -1.13095(9) & -0.15114(7) & -0.82300(7) & 1.90(2) \\ \mathrm{P}(1) & -0.8636(1) & -0.3176(1) & -0.78920(10) & 1.43(3) \\ \mathrm{P}(2) & -0.8206(2) & -0.2302(1) & -0.5511(1) & 1.64(3) \\ \mathrm{O}(1) & -0.8012(8) & 0.1275(6) & -0.9032(8) & 8.8(3) \\ \mathrm{C}(1) & -0.6591(6) & -0.4483(4) & -0.7323(4) & 1.9(1) \\ \mathrm{C}(2) & -0.5779(6) & -0.4615(5) & -0.8146(5) & 2.3(1) \\ \mathrm{C}(3) & -0.5545(6) & -0.5456(5) & -0.8500(5) & 2.7(1) \\ \mathrm{C}(4) & -0.6112(7) & -0.6183(5) & -0.8035(5) & 2.7(1) \\ \mathrm{C}(5) & -0.6903(7) & -0.6056(5) & -0.7194(5) & 2.7(1) \\ \mathrm{C}(6) & -0.7127(6) & -0.5214(5) & -0.6869(5) & 2.4(1) \\ \mathrm{C}(7) & -0.7867(5) & -0.3118(4) & -0.9119(4) & 1.7(1) \\ \mathrm{C}(8) & -0.6571(6) & -0.2694(5) & -0.9197(5) & 2.3(1) \\ \mathrm{C}(9) & -0.6071(6) & -0.2512(5) & -1.0124(5) & 2.7(1) \\ \mathrm{C}(10) & -0.6841(7) & -0.2764(6) & -1.0968(5) & 3.2(2) \\ \mathrm{C}(11) & -0.8113(7) & -0.3179(6) & -1.0886(4) & 3.0(2) \\ \mathrm{C}(12) & -0.8654(5) & -0.3345(6) & -0.9965(4) & 2.2(1) \\ \mathrm{C}(13) & -0.9969(6) & -0.4038(4) & -0.7997(4) & 1.7(1) \\ \mathrm{C}(14) & -0.9638(6) & -0.4829(5) & -0.8472(5) & 2.1(1) \\ \mathrm{C}(15) & -1.0606(7) & -0.5507(5) & -0.8516(5) & 2.6(1) \\ \mathrm{C}(16) & -1.1881(7) & -0.5423(5) & -0.8076(5) & 2.5(1) \\ \mathrm{C}(17) & -1.2196(6) & -0.4671(5) & -0.7564(5) & 2.4(1) \\ \mathrm{C}(18) & -1.1246(6) & -0.3968(5) & -0.7519(4) & 1.9(1) \\ \mathrm{C}(19) & -0.9400(5) & -0.2077(4) & -0.7889(4) & 1.4(1) \\ \mathrm{C}(20) & -0.9285(6) & -0.1440(5) & -0.8660(4) & 2.3(1) \\ \mathrm{C}(21) & -0.9710(6) & -0.0615(5) & -0.8299(5) & 2.5(1) \\ \mathrm{C}(22) & -1.0111(6) & -0.0720(5) & -0.7307(5) & 2.5(1) \\ \mathrm{C}(23) & -0.9889(5) & -0.1618(4) & -0.7030(4) & 1.6(1) \\ \mathrm{C}(24) & -1.3226(6) & -0.2054(6) & -0.7951(6) & 3.2(2) \\ \mathrm{C}(25) & -1.2694(7) & -0.2395(5) & -0.8844(5) & 3.0(2) \\ \mathrm{C}(26) & -1.2509(7) & -0.1686(6) & -0.9501(5) & 3.1(2) \\ & & & & \end{array}$


Table S6. Atomic coordinates and $\mathrm{B}_{\mathrm{iso}} / \mathrm{B}_{\text {eq }}$ for $\mathbf{5}$ (continued)

$\begin{array}{lllll}\text { atom } & \mathrm{x} & \mathrm{y} & \mathrm{z} & \mathrm{B} \text { eq } \\ & & & & \\ \mathrm{C}(27) & -1.2900(7) & -0.0891(6) & -0.9014(6) & 3.5(2) \\ \mathrm{C}(28) & -1.3365(6) & -0.1133(6) & -0.8062(6) & 3.4(2) \\ \mathrm{C}(29) & -1.0030(6) & -0.2033(5) & -0.6036(4) & 2.0(1) \\ \mathrm{C}(30) & -1.1137(7) & -0.1543(6) & -0.5424(5) & 2.9(1) \\ \mathrm{C}(31) & -0.8620(7) & -0.2869(5) & -0.4318(4) & 2.3(1) \\ \mathrm{C}(32) & -0.7340(7) & -0.3404(6) & -0.3921(4) & 3.1(1) \\ \mathrm{C}(33) & -0.9786(7) & -0.3556(6) & -0.4561(4) & 3.2(2) \\ \mathrm{C}(34) & -0.9097(8) & -0.2267(6) & -0.3481(5) & 3.2(2) \\ \mathrm{C}(35) & -0.7189(6) & -0.1251(4) & -0.5287(5) & 2.2(1) \\ \mathrm{C}(36) & -0.6508(7) & -0.1033(5) & -0.6268(5) & 2.9(2) \\ \mathrm{C}(37) & -0.8074(7) & -0.0464(5) & -0.4989(5) & 2.6(1) \\ \mathrm{C}(38) & -0.5981(7) & -0.1376(5) & -0.4511(5) & 3.0(2) \\ \mathrm{C}(40) & -0.791(1) & 0.148(1) & -0.801(1) & 10.7(5) \\ \mathrm{C}(41) & -0.644(2) & 0.122(1) & -0.768(1) & 9.6(5) \\ \mathrm{C}(42) & -0.6103(10) & 0.0545(9) & -0.8455(10) & 6.3(3) \\ \mathrm{C}(43) & -0.692(1) & 0.079(1) & -0.9312(8) & 7.4(4) \\ \mathrm{H}(1) & -0.5379 & -0.4123 & -0.8469 & 2.7806 \\ \mathrm{H}(2) & -0.4991 & -0.5536 & -0.9067 & 3.1831 \\ \mathrm{H}(3) & -0.5963 & -0.6758 & -0.8285 & 3.2465 \\ \mathrm{H}(4) & -0.7280 & -0.6545 & -0.6853 & 3.2688 \\ \mathrm{H}(5) & -0.7679 & -0.5131 & -0.6302 & 2.9095 \\ \mathrm{H}(6) & -0.6039 & -0.2533 & -0.8619 & 2.7150 \\ \mathrm{H}(7) & -0.5199 & -0.2213 & -1.0185 & 3.2522 \\ \mathrm{H}(8) & -0.6484 & -0.2648 & -1.1604 & 3.8276 \\ \mathrm{H}(9) & -0.8628 & -0.3355 & -1.1465 & 3.6418 \\ \mathrm{H}(10) & -0.9554 & -0.3611 & -0.9912 & 2.5980 \\ \mathrm{H}(11) & -0.8746 & -0.4899 & -0.8763 & 2.5574 \\ \mathrm{H}(12) & -1.0387 & -0.6035 & -0.8855 & 3.1049 \\ \mathrm{H}(13) & -1.2551 & -0.5887 & -0.8126 & 2.9874 \\ \mathrm{H}(14) & -1.3066 & -0.4629 & -0.7237 & 2.8469 \\ \mathrm{H}(15) & -1.1467 & -0.3448 & -0.7167 & 2.3329 \\ \mathrm{H}(16) & -0.8973 & -0.1554 & -0.9308 & 2.7224\end{array}$


Table S6. Atomic coordinates and $\mathrm{B}_{\mathrm{iso}} / \mathrm{B}_{\mathrm{eq}}$ for $\mathbf{5}$ (continued)

$\begin{array}{lllll}\text { atom } & \mathrm{x} & \mathrm{y} & \mathrm{z} & \mathrm{B} \text { eq } \\ & & & & \\ \mathrm{H}(17) & -0.9725 & -0.0079 & -0.8660 & 3.0102 \\ \mathrm{H}(18) & -1.0467 & -0.0270 & -0.6896 & 3.0004 \\ \mathrm{H}(19) & -1.3448 & -0.2386 & -0.7381 & 3.8296 \\ \mathrm{H}(20) & -1.2496 & -0.2996 & -0.8975 & 3.6361 \\ \mathrm{H}(21) & -1.2180 & -0.1729 & -1.0155 & 3.7129 \\ \mathrm{H}(22) & -1.2859 & -0.0312 & -0.9276 & 4.1909 \\ \mathrm{H}(23) & -1.3711 & -0.0740 & -0.7580 & 4.0198 \\ \mathrm{H}(24) & -1.0445 & -0.2591 & -0.6172 & 2.3488 \\ \mathrm{H}(25) & -1.0804 & -0.0969 & -0.5268 & 3.4853 \\ \mathrm{H}(26) & -1.1291 & -0.1858 & -0.4831 & 3.4853 \\ \mathrm{H}(27) & -1.1998 & -0.1502 & -0.5799 & 3.4853 \\ \mathrm{H}(28) & -0.7059 & -0.3810 & -0.4412 & 3.7110 \\ \mathrm{H}(29) & -0.7592 & -0.3716 & -0.3344 & 3.7110 \\ \mathrm{H}(30) & -0.6583 & -0.3015 & -0.3764 & 3.7110 \\ \mathrm{H}(31) & -1.0621 & -0.3262 & -0.4780 & 3.8230 \\ \mathrm{H}(32) & -0.9972 & -0.3889 & -0.3985 & 3.8230 \\ \mathrm{H}(33) & -0.9481 & -0.3939 & -0.5066 & 3.8230 \\ \mathrm{H}(34) & -0.8361 & -0.1867 & -0.3306 & 3.8831 \\ \mathrm{H}(35) & -0.9317 & -0.2614 & -0.2922 & 3.8831 \\ \mathrm{H}(36) & -0.9909 & -0.1946 & -0.3696 & 3.8831 \\ \mathrm{H}(37) & -0.5924 & -0.1509 & -0.6456 & 3.4351 \\ \mathrm{H}(38) & -0.5955 & -0.0515 & -0.6193 & 3.4351 \\ \mathrm{H}(39) & -0.7223 & -0.0941 & -0.6763 & 3.4351 \\ \mathrm{H}(40) & -0.7485 & 0.0039 & -0.4910 & 3.1358 \\ \mathrm{H}(41) & -0.8512 & -0.0587 & -0.4382 & 3.1358 \\ \mathrm{H}(42) & -0.8776 & -0.0352 & -0.5487 & 3.1358 \\ \mathrm{H}(43) & -0.5409 & -0.1858 & -0.4697 & 3.6022 \\ \mathrm{H}(44) & -0.6365 & -0.1491 & -0.3884 & 3.6022 \\ \mathrm{H}(45) & -0.5426 & -0.0856 & -0.4474 & 3.6022 \\ \mathrm{H}(46) & -0.8586 & 0.1162 & -0.7650 & 12.8082 \\ \mathrm{H}(47) & -0.8050 & 0.2097 & -0.7907 & 12.8082 \\ \mathrm{H}(48) & -0.6415 & 0.0974 & -0.7034 & 11.5774\end{array}$


Table S6. Atomic coordinates and $\mathrm{B}_{\mathrm{iso}} / \mathrm{B}_{\mathrm{eq}}$ for $\mathbf{5}$ (continued)

$\begin{array}{lcccl}\text { atom } & \mathrm{x} & \mathrm{y} & \mathrm{z} & \mathrm{B} \text { eq } \\ & & & & \\ \mathrm{H}(49) & -0.5805 & 0.1708 & -0.7694 & 11.5774 \\ \mathrm{H}(50) & -0.6357 & -0.0027 & -0.8239 & 7.6082 \\ \mathrm{H}(51) & -0.5128 & 0.0556 & -0.8592 & 7.6082 \\ \mathrm{H}(52) & -0.6360 & 0.1115 & -0.9747 & 8.8949 \\ \mathrm{H}(53) & -0.7261 & 0.0271 & -0.9639 & 8.8949 \\ \mathrm{~B} \text { eq }=8 / 3 \pi^{2}\left(\mathrm{U}_{11}(\mathrm{aa})^{2}+\mathrm{U}_{22}\left(\mathrm{bb}^{*}\right)^{2}+\mathrm{U}_{33}\left(\mathrm{cc}^{*}\right)^{2}+2 \mathrm{U}_{12}\left(\mathrm{aa}^{*} \mathrm{bb}\right) \cos \gamma+2 \mathrm{U}_{13}\left(\mathrm{aa}^{*} \mathrm{cc} *\right) \cos \beta+\right. \\ \left.2 \mathrm{U}_{23}\left(\mathrm{bb}^{*} \mathrm{cc} *\right) \cos \alpha\right)\end{array}$


Table S7. Anisotropic Displacement Parameters for 5

\begin{tabular}{|c|c|c|c|c|c|c|}
\hline atom & $\mathrm{U}_{11}$ & $\mathrm{U}_{22}$ & $\mathrm{U}_{33}$ & $\mathrm{U}_{12}$ & $\mathrm{U}_{13}$ & $\mathrm{U}_{23}$ \\
\hline $\operatorname{Pd}(1)$ & $0.0191(2)$ & $0.0238(2)$ & $0.0162(2)$ & $0.0025(2)$ & $-0.0023(1)$ & $-0.0011(2)$ \\
\hline $\operatorname{Br}(1)$ & $0.0278(3)$ & $0.0442(5)$ & $0.0333(3)$ & $0.0100(3)$ & $-0.0104(2)$ & $-0.0066(3)$ \\
\hline $\mathrm{Fe}(1)$ & $0.0207(4)$ & $0.0269(5)$ & $0.0242(5)$ & $0.0035(4)$ & $-0.0048(3)$ & $0.0021(4)$ \\
\hline $\mathrm{P}(1)$ & $0.0149(6)$ & $0.0238(9)$ & $0.0155(6)$ & $0.0000(6)$ & $0.0000(5)$ & $0.0002(6)$ \\
\hline $\mathrm{P}(2)$ & $0.0252(7)$ & $0.0207(8)$ & $0.0163(7)$ & $0.0008(6)$ & $-0.0020(6)$ & $-0.0010(6)$ \\
\hline $\mathrm{O}(1)$ & $0.062(5)$ & $0.092(7)$ & $0.181(9)$ & $0.029(4)$ & $0.018(5)$ & $0.060(7)$ \\
\hline $\mathrm{C}(1)$ & $0.023(3)$ & $0.030(4)$ & $0.020(3)$ & $0.006(3)$ & $-0.003(2)$ & $0.000(3)$ \\
\hline$C(2)$ & $0.017(3)$ & $0.036(4)$ & $0.034(4)$ & $0.004(3)$ & $-0.004(2)$ & $-0.005(3)$ \\
\hline$C(3)$ & $0.026(3)$ & $0.042(5)$ & $0.032(3)$ & $0.013(3)$ & $-0.003(3)$ & $-0.014(3)$ \\
\hline $\mathrm{C}(4)$ & $0.036(3)$ & $0.034(4)$ & $0.032(4)$ & $0.008(3)$ & $-0.008(3)$ & $-0.013(3)$ \\
\hline$C(5)$ & $0.042(4)$ & $0.027(4)$ & $0.034(4)$ & $0.007(3)$ & $-0.001(3)$ & $-0.001(3)$ \\
\hline$C(6)$ & $0.037(3)$ & $0.027(4)$ & $0.028(3)$ & $0.002(3)$ & $0.002(3)$ & $-0.007(3)$ \\
\hline$C(7)$ & $0.022(3)$ & $0.028(4)$ & $0.015(2)$ & $0.000(2)$ & $0.000(2)$ & $-0.002(2)$ \\
\hline $\mathrm{C}(8)$ & $0.016(3)$ & $0.041(4)$ & $0.028(3)$ & $0.001(3)$ & $-0.005(2)$ & $0.001(3)$ \\
\hline $\mathrm{C}(9)$ & $0.028(3)$ & $0.044(5)$ & $0.032(3)$ & $-0.001(3)$ & $0.006(3)$ & $0.013(3)$ \\
\hline$C(10)$ & $0.036(4)$ & $0.060(5)$ & $0.026(3)$ & $0.000(4)$ & $0.007(3)$ & $0.007(4)$ \\
\hline$C(11)$ & $0.044(3)$ & $0.055(6)$ & $0.016(3)$ & $-0.002(4)$ & $-0.004(2)$ & $-0.001(3)$ \\
\hline $\mathrm{C}(12)$ & $0.030(3)$ & $0.033(3)$ & $0.019(2)$ & $-0.006(3)$ & $0.001(2)$ & $-0.002(4)$ \\
\hline$C(13)$ & $0.023(3)$ & $0.024(3)$ & $0.016(3)$ & $0.000(2)$ & $-0.003(2)$ & $0.004(2)$ \\
\hline $\mathrm{C}(14)$ & $0.022(3)$ & $0.030(4)$ & $0.029(3)$ & $0.001(3)$ & $0.003(2)$ & $-0.002(3)$ \\
\hline$C(15)$ & $0.043(4)$ & $0.022(3)$ & $0.033(4)$ & $0.002(3)$ & $-0.004(3)$ & $0.000(3)$ \\
\hline$C(16)$ & $0.032(3)$ & $0.031(4)$ & $0.032(3)$ & $-0.005(3)$ & $-0.008(3)$ & $0.008(3)$ \\
\hline$C(17)$ & $0.019(3)$ & $0.043(4)$ & $0.028(3)$ & $-0.009(3)$ & $-0.002(2)$ & $0.006(3)$ \\
\hline $\mathrm{C}(18)$ & $0.019(3)$ & $0.034(4)$ & $0.021(3)$ & $-0.002(3)$ & $-0.002(2)$ & $0.003(3)$ \\
\hline C(19) & $0.015(2)$ & $0.017(3)$ & $0.022(3)$ & $0.000(2)$ & $-0.004(2)$ & $0.005(2)$ \\
\hline $\mathrm{C}(20)$ & $0.022(3)$ & $0.037(4)$ & $0.026(3)$ & $0.002(3)$ & $0.002(2)$ & $0.001(3)$ \\
\hline $\mathrm{C}(21)$ & $0.029(3)$ & $0.028(4)$ & $0.038(4)$ & $-0.006(3)$ & $-0.009(3)$ & $0.008(3)$ \\
\hline $\mathrm{C}(22)$ & $0.030(3)$ & $0.026(4)$ & $0.039(4)$ & $0.001(3)$ & $-0.007(3)$ & $0.001(3)$ \\
\hline$C(23)$ & $0.021(3)$ & $0.019(3)$ & $0.022(3)$ & $0.002(2)$ & $-0.004(2)$ & $-0.002(3)$ \\
\hline$C(24)$ & $0.017(3)$ & $0.055(5)$ & $0.049(4)$ & $-0.002(3)$ & $-0.005(3)$ & $0.011(4)$ \\
\hline$C(25)$ & $0.030(3)$ & $0.039(4)$ & $0.045(4)$ & $-0.003(3)$ & $-0.019(3)$ & $0.002(4)$ \\
\hline$C(26)$ & $0.038(4)$ & $0.052(5)$ & $0.027(3)$ & $-0.002(4)$ & $-0.011(3)$ & $-0.003(4)$ \\
\hline
\end{tabular}


Table S7. Anisotropic Displacement Parameters for 5 (continued)

$\begin{array}{lllllll}\text { atom } & \mathrm{U} 11 & \mathrm{U} 22 & \mathrm{U} 33 & \mathrm{U} 12 & \mathrm{U} 13 & \mathrm{U} 23 \\ \mathrm{C}(27) & 0.029(3) & 0.057(5) & 0.046(4) & 0.004(3) & -0.020(3) & 0.019(4) \\ \mathrm{C}(28) & 0.018(3) & 0.058(6) & 0.051(5) & 0.014(3) & -0.002(3) & 0.002(4) \\ \mathrm{C}(29) & 0.026(3) & 0.027(3) & 0.021(3) & 0.000(3) & 0.001(2) & -0.008(3) \\ \mathrm{C}(30) & 0.033(3) & 0.045(5) & 0.032(4) & 0.014(3) & -0.001(3) & -0.007(3) \\ \mathrm{C}(31) & 0.039(3) & 0.027(4) & 0.020(3) & -0.005(3) & -0.003(3) & 0.007(3) \\ \mathrm{C}(32) & 0.057(4) & 0.036(4) & 0.024(3) & 0.008(4) & 0.000(3) & 0.014(4) \\ \mathrm{C}(33) & 0.052(4) & 0.043(5) & 0.027(3) & -0.015(4) & 0.007(3) & 0.003(3) \\ \mathrm{C}(34) & 0.057(5) & 0.045(5) & 0.021(3) & 0.003(4) & -0.003(3) & 0.002(3) \\ \mathrm{C}(35) & 0.031(3) & 0.023(3) & 0.029(3) & -0.005(3) & -0.007(2) & 0.000(3) \\ \mathrm{C}(36) & 0.039(4) & 0.028(4) & 0.042(4) & -0.008(3) & -0.006(3) & 0.008(3) \\ \mathrm{C}(37) & 0.038(4) & 0.021(4) & 0.039(4) & 0.000(3) & -0.012(3) & -0.004(3) \\ \mathrm{C}(38) & 0.033(3) & 0.038(4) & 0.043(4) & -0.003(3) & -0.012(3) & -0.007(4) \\ \mathrm{C}(40) & 0.077(8) & 0.09(1) & 0.24(2) & -0.049(8) & 0.08(1) & -0.09(1) \\ \mathrm{C}(41) & 0.17(1) & 0.10(1) & 0.096(10) & -0.03(1) & -0.014(9) & 0.049(9) \\ \mathrm{C}(42) & 0.057(6) & 0.078(8) & 0.107(9) & 0.024(5) & 0.024(6) & 0.041(7) \\ \mathrm{C}(43) & 0.054(6) & 0.15(1) & 0.082(8) & -0.006(7) & 0.002(5) & 0.050(8)\end{array}$

The general temperature factor expression:

$\exp \left(-2 \pi^{2}\left(a^{*}{ }^{2} U_{11} h^{2}+b^{* 2} U_{22} k^{2}+c^{* 2} U_{33} l^{2}+2 a * b * U_{12} h k+2 a * c * U_{13} h l+2 b * c * U_{23} k l\right)\right)$ 
Table S8. Bond Lengths $(\AA)$ for 5

\begin{tabular}{|c|c|c|c|c|c|}
\hline atom & atom & distance & atom & atom & distance \\
\hline $\operatorname{Pd}(1)$ & $\operatorname{Br}(1)$ & $2.5301(6)$ & $\operatorname{Pd}(1)$ & $\mathrm{P}(1)$ & $2.240(1)$ \\
\hline $\operatorname{Pd}(1)$ & $\mathrm{P}(2)$ & $2.436(2)$ & $\operatorname{Pd}(1)$ & $\mathrm{C}(1)$ & $2.045(6)$ \\
\hline $\mathrm{Fe}(1)$ & $\mathrm{C}(19)$ & $2.046(5)$ & $\mathrm{Fe}(1)$ & $\mathrm{C}(20)$ & $2.028(6)$ \\
\hline $\mathrm{Fe}(1)$ & $\mathrm{C}(21)$ & $2.043(6)$ & $\mathrm{Fe}(1)$ & $\mathrm{C}(22)$ & $2.053(7)$ \\
\hline $\mathrm{Fe}(1)$ & $\mathrm{C}(23)$ & $2.086(5)$ & $\mathrm{Fe}(1)$ & $\mathrm{C}(24)$ & $2.043(7)$ \\
\hline $\mathrm{Fe}(1)$ & $C(25)$ & $2.038(7)$ & $\mathrm{Fe}(1)$ & $C(26)$ & $2.052(6)$ \\
\hline $\mathrm{Fe}(1)$ & $\mathrm{C}(27)$ & $2.049(7)$ & $\mathrm{Fe}(1)$ & $\mathrm{C}(28)$ & $2.053(6)$ \\
\hline $\mathrm{P}(1)$ & $\mathrm{C}(7)$ & $1.837(5)$ & $\mathrm{P}(1)$ & $\mathrm{C}(13)$ & $1.823(6)$ \\
\hline $\mathrm{P}(1)$ & $\mathrm{C}(19)$ & $1.821(6)$ & $\mathrm{P}(2)$ & $\mathrm{C}(29)$ & $1.896(6)$ \\
\hline $\mathrm{P}(2)$ & $\mathrm{C}(31)$ & $1.882(6)$ & $\mathrm{P}(2)$ & $\mathrm{C}(35)$ & $1.886(7)$ \\
\hline $\mathrm{O}(1)$ & $\mathrm{C}(40)$ & $1.42(2)$ & $\mathrm{O}(1)$ & $\mathrm{C}(43)$ & $1.34(1)$ \\
\hline$C(1)$ & $\mathrm{C}(2)$ & $1.387(9)$ & $\mathrm{C}(1)$ & $C(6)$ & $1.374(10)$ \\
\hline$C(2)$ & $\mathrm{C}(3)$ & $1.385(10)$ & $\mathrm{C}(3)$ & $\mathrm{C}(4)$ & $1.39(1)$ \\
\hline$C(4)$ & $C(5)$ & $1.395(9)$ & $C(5)$ & $C(6)$ & $1.372(10)$ \\
\hline$C(7)$ & $\mathrm{C}(8)$ & $1.394(8)$ & $C(7)$ & $\mathrm{C}(12)$ & $1.392(7)$ \\
\hline$C(8)$ & $\mathrm{C}(9)$ & $1.383(9)$ & $\mathrm{C}(9)$ & $C(10)$ & $1.392(10)$ \\
\hline$C(10)$ & $\mathrm{C}(11)$ & $1.369(9)$ & $\mathrm{C}(11)$ & $\mathrm{C}(12)$ & $1.385(8)$ \\
\hline $\mathrm{C}(13)$ & $C(14)$ & $1.403(9)$ & $\mathrm{C}(13)$ & $\mathrm{C}(18)$ & $1.395(8)$ \\
\hline $\mathrm{C}(14)$ & $\mathrm{C}(15)$ & $1.380(9)$ & $\mathrm{C}(15)$ & $C(16)$ & $1.371(9)$ \\
\hline$C(16)$ & $\mathrm{C}(17)$ & $1.37(1)$ & $\mathrm{C}(17)$ & $\mathrm{C}(18)$ & $1.398(9)$ \\
\hline$C(19)$ & $\mathrm{C}(20)$ & $1.429(9)$ & $\mathrm{C}(19)$ & $\mathrm{C}(23)$ & $1.444(8)$ \\
\hline $\mathrm{C}(20)$ & $\mathrm{C}(21)$ & $1.41(1)$ & $\mathrm{C}(21)$ & $\mathrm{C}(22)$ & $1.415(9)$ \\
\hline$C(22)$ & $\mathrm{C}(23)$ & $1.429(9)$ & $\mathrm{C}(23)$ & C(29) & $1.495(8)$ \\
\hline$C(24)$ & $\mathrm{C}(25)$ & $1.42(1)$ & $\mathrm{C}(24)$ & $\mathrm{C}(28)$ & $1.41(1)$ \\
\hline$C(25)$ & $C(26)$ & $1.41(1)$ & $C(26)$ & $\mathrm{C}(27)$ & $1.43(1)$ \\
\hline$C(27)$ & $\mathrm{C}(28)$ & $1.42(1)$ & $\mathrm{C}(29)$ & $C(30)$ & $1.548(8)$ \\
\hline $\mathrm{C}(31)$ & $\mathrm{C}(32)$ & $1.544(9)$ & $\mathrm{C}(31)$ & $\mathrm{C}(33)$ & $1.549(9)$ \\
\hline$C(31)$ & $C(34)$ & $1.536(10)$ & $C(35)$ & $C(36)$ & $1.530(9)$ \\
\hline $\mathrm{C}(35)$ & $\mathrm{C}(37)$ & $1.523(10)$ & $C(35)$ & $\mathrm{C}(38)$ & $1.543(9)$ \\
\hline $\mathrm{C}(40)$ & $\mathrm{C}(41)$ & $1.51(2)$ & $\mathrm{C}(41)$ & $\mathrm{C}(42)$ & $1.51(2)$ \\
\hline$C(42)$ & $\mathrm{C}(43)$ & $1.42(1)$ & & & \\
\hline
\end{tabular}


Table S9. Bond Lengths for the Hydrogen Atoms (A) for 5

\begin{tabular}{|c|c|c|c|c|c|}
\hline atom & atom & distance & atom & atom & distance \\
\hline$C(2)$ & $\mathrm{H}(1)$ & 0.95 & $\mathrm{C}(3)$ & $\mathrm{H}(2)$ & 0.95 \\
\hline $\mathrm{C}(4)$ & $\mathrm{H}(3)$ & 0.95 & $C(5)$ & $\mathrm{H}(4)$ & 0.95 \\
\hline$C(6)$ & $\mathrm{H}(5)$ & 0.95 & $\mathrm{C}(8)$ & $\mathrm{H}(6)$ & 0.95 \\
\hline $\mathrm{C}(9)$ & $\mathrm{H}(7)$ & 0.95 & $\mathrm{C}(10)$ & $\mathrm{H}(8)$ & 0.95 \\
\hline $\mathrm{C}(11)$ & $\mathrm{H}(9)$ & 0.95 & $\mathrm{C}(12)$ & $\mathrm{H}(10)$ & 0.95 \\
\hline $\mathrm{C}(14)$ & $\mathrm{H}(11)$ & 0.95 & $\mathrm{C}(15)$ & $\mathrm{H}(12)$ & 0.95 \\
\hline$C(16)$ & $\mathrm{H}(13)$ & 0.95 & $\mathrm{C}(17)$ & $\mathrm{H}(14)$ & 0.95 \\
\hline $\mathrm{C}(18)$ & $\mathrm{H}(15)$ & 0.95 & $\mathrm{C}(20)$ & $\mathrm{H}(16)$ & 0.95 \\
\hline $\mathrm{C}(21)$ & $\mathrm{H}(17)$ & 0.95 & $\mathrm{C}(22)$ & $\mathrm{H}(18)$ & 0.95 \\
\hline$C(24)$ & $\mathrm{H}(19)$ & 0.95 & $\mathrm{C}(25)$ & $\mathrm{H}(20)$ & 0.95 \\
\hline$C(26)$ & $\mathrm{H}(21)$ & 0.95 & $\mathrm{C}(27)$ & $\mathrm{H}(22)$ & 0.95 \\
\hline $\mathrm{C}(28)$ & $\mathrm{H}(23)$ & 0.95 & $\mathrm{C}(29)$ & $\mathrm{H}(24)$ & 0.95 \\
\hline $\mathrm{C}(30)$ & $\mathrm{H}(25)$ & 0.95 & $\mathrm{C}(30)$ & $\mathrm{H}(26)$ & 0.95 \\
\hline$C(30)$ & $\mathrm{H}(27)$ & 0.95 & $\mathrm{C}(32)$ & $\mathrm{H}(28)$ & 0.95 \\
\hline$C(32)$ & $\mathrm{H}(29)$ & 0.95 & $\mathrm{C}(32)$ & $\mathrm{H}(30)$ & 0.95 \\
\hline $\mathrm{C}(33)$ & $\mathrm{H}(31)$ & 0.95 & $\mathrm{C}(33)$ & $\mathrm{H}(32)$ & 0.95 \\
\hline $\mathrm{C}(33)$ & $\mathrm{H}(33)$ & 0.95 & $\mathrm{C}(34)$ & $\mathrm{H}(34)$ & 0.95 \\
\hline $\mathrm{C}(34)$ & $\mathrm{H}(35)$ & 0.95 & $\mathrm{C}(34)$ & $\mathrm{H}(36)$ & 0.95 \\
\hline$C(36)$ & $\mathrm{H}(37)$ & 0.95 & $\mathrm{C}(36)$ & $\mathrm{H}(38)$ & 0.95 \\
\hline$C(36)$ & $\mathrm{H}(39)$ & 0.95 & $\mathrm{C}(37)$ & $\mathrm{H}(40)$ & 0.95 \\
\hline $\mathrm{C}(37)$ & $\mathrm{H}(41)$ & 0.95 & $\mathrm{C}(37)$ & $\mathrm{H}(42)$ & 0.95 \\
\hline $\mathrm{C}(38)$ & $\mathrm{H}(43)$ & 0.95 & $\mathrm{C}(38)$ & $\mathrm{H}(44)$ & 0.95 \\
\hline $\mathrm{C}(38)$ & $\mathrm{H}(45)$ & 0.95 & $\mathrm{C}(40)$ & $\mathrm{H}(46)$ & 0.95 \\
\hline $\mathrm{C}(40)$ & $\mathrm{H}(47)$ & 0.95 & $\mathrm{C}(41)$ & $\mathrm{H}(48)$ & 0.95 \\
\hline $\mathrm{C}(41)$ & $\mathrm{H}(49)$ & 0.95 & $\mathrm{C}(42)$ & $\mathrm{H}(50)$ & 0.95 \\
\hline $\mathrm{C}(42)$ & $\mathrm{H}(51)$ & 0.95 & $\mathrm{C}(43)$ & $\mathrm{H}(52)$ & 0.95 \\
\hline $\mathrm{C}(43)$ & $\mathrm{H}(53)$ & 0.95 & & & \\
\hline
\end{tabular}


Table S10. Bond Angles $\left({ }^{\circ}\right)$ for 5

\begin{tabular}{|c|c|c|c|c|c|c|c|}
\hline atom & atom & atom & angle & atom & atom & atom & angle \\
\hline $\operatorname{Br}(1)$ & $\operatorname{Pd}(1)$ & $\mathrm{P}(1)$ & $157.94(4)$ & $\operatorname{Br}(1)$ & $\operatorname{Pd}(1)$ & $\mathrm{P}(2)$ & $98.97(4)$ \\
\hline $\operatorname{Br}(1)$ & $\operatorname{Pd}(1)$ & $\mathrm{C}(1)$ & $86.1(2)$ & $\mathrm{P}(1)$ & $\operatorname{Pd}(1)$ & $\mathrm{P}(2)$ & $96.73(5)$ \\
\hline $\mathrm{P}(1)$ & $\operatorname{Pd}(1)$ & $\mathrm{C}(1)$ & $83.8(2)$ & $\mathrm{P}(2)$ & $\operatorname{Pd}(1)$ & $\mathrm{C}(1)$ & $161.5(2)$ \\
\hline C(19) & $\mathrm{Fe}(1)$ & $\mathrm{C}(20)$ & $41.1(2)$ & $\mathrm{C}(19)$ & $\mathrm{Fe}(1)$ & $\mathrm{C}(21)$ & $68.6(2)$ \\
\hline C(19) & $\mathrm{Fe}(1)$ & $\mathrm{C}(22)$ & $68.7(2)$ & $\mathrm{C}(19)$ & $\mathrm{Fe}(1)$ & $\mathrm{C}(23)$ & $40.9(2)$ \\
\hline C(19) & $\mathrm{Fe}(1)$ & $\mathrm{C}(24)$ & $125.2(3)$ & $\mathrm{C}(19)$ & $\mathrm{Fe}(1)$ & $\mathrm{C}(25)$ & $111.8(3)$ \\
\hline $\mathrm{C}(19)$ & $\mathrm{Fe}(1)$ & $C(26)$ & $126.9(3)$ & $\mathrm{C}(19)$ & $\mathrm{Fe}(1)$ & $\mathrm{C}(27)$ & $160.8(3)$ \\
\hline C(19) & $\mathrm{Fe}(1)$ & $\mathrm{C}(28)$ & $158.6(3)$ & $\mathrm{C}(20)$ & $\mathrm{Fe}(1)$ & $\mathrm{C}(21)$ & $40.5(3)$ \\
\hline $\mathrm{C}(20)$ & $\mathrm{Fe}(1)$ & $\mathrm{C}(22)$ & $68.3(3)$ & $\mathrm{C}(20)$ & $\mathrm{Fe}(1)$ & $\mathrm{C}(23)$ & $68.4(2)$ \\
\hline $\mathrm{C}(20)$ & $\mathrm{Fe}(1)$ & $\mathrm{C}(24)$ & $158.7(3)$ & $\mathrm{C}(20)$ & $\mathrm{Fe}(1)$ & $\mathrm{C}(25)$ & $121.6(3)$ \\
\hline $\mathrm{C}(20)$ & $\mathrm{Fe}(1)$ & $C(26)$ & $105.9(3)$ & $\mathrm{C}(20)$ & $\mathrm{Fe}(1)$ & $\mathrm{C}(27)$ & 121.1(3) \\
\hline $\mathrm{C}(20)$ & $\mathrm{Fe}(1)$ & $\mathrm{C}(28)$ & $158.3(3)$ & $\mathrm{C}(21)$ & $\mathrm{Fe}(1)$ & $C(22)$ & $40.4(3)$ \\
\hline $\mathrm{C}(21)$ & $\mathrm{Fe}(1)$ & $C(23)$ & $67.9(2)$ & $\mathrm{C}(21)$ & $\mathrm{Fe}(1)$ & $\mathrm{C}(24)$ & $160.8(3)$ \\
\hline $\mathrm{C}(21)$ & $\mathrm{Fe}(1)$ & $\mathrm{C}(25)$ & $153.0(3)$ & $\mathrm{C}(21)$ & $\mathrm{Fe}(1)$ & $C(26)$ & $116.3(3)$ \\
\hline $\mathrm{C}(21)$ & $\mathrm{Fe}(1)$ & $\mathrm{C}(27)$ & $102.0(3)$ & $\mathrm{C}(21)$ & $\mathrm{Fe}(1)$ & $\mathrm{C}(28)$ & $121.9(3)$ \\
\hline $\mathrm{C}(22)$ & $\mathrm{Fe}(1)$ & $\mathrm{C}(23)$ & $40.4(3)$ & $\mathrm{C}(22)$ & $\mathrm{Fe}(1)$ & $\mathrm{C}(24)$ & $127.2(3)$ \\
\hline $\mathrm{C}(22)$ & $\mathrm{Fe}(1)$ & $\mathrm{C}(25)$ & $166.5(3)$ & $\mathrm{C}(22)$ & $\mathrm{Fe}(1)$ & $C(26)$ & $150.4(3)$ \\
\hline $\mathrm{C}(22)$ & $\mathrm{Fe}(1)$ & $\mathrm{C}(27)$ & $115.6(3)$ & $\mathrm{C}(22)$ & $\mathrm{Fe}(1)$ & $\mathrm{C}(28)$ & $106.3(3)$ \\
\hline $\mathrm{C}(23)$ & $\mathrm{Fe}(1)$ & $\mathrm{C}(24)$ & $112.5(3)$ & $\mathrm{C}(23)$ & $\mathrm{Fe}(1)$ & $C(25)$ & $131.3(3)$ \\
\hline $\mathrm{C}(23)$ & $\mathrm{Fe}(1)$ & $C(26)$ & $166.5(3)$ & $\mathrm{C}(23)$ & $\mathrm{Fe}(1)$ & $\mathrm{C}(27)$ & $152.7(3)$ \\
\hline$C(23)$ & $\mathrm{Fe}(1)$ & $\mathrm{C}(28)$ & $122.0(3)$ & $\mathrm{C}(24)$ & $\mathrm{Fe}(1)$ & $\mathrm{C}(25)$ & $40.8(3)$ \\
\hline$C(24)$ & $\mathrm{Fe}(1)$ & $C(26)$ & $68.2(3)$ & $\mathrm{C}(24)$ & $\mathrm{Fe}(1)$ & $\mathrm{C}(27)$ & $68.6(3)$ \\
\hline $\mathrm{C}(24)$ & $\mathrm{Fe}(1)$ & $\mathrm{C}(28)$ & $40.3(3)$ & $\mathrm{C}(25)$ & $\mathrm{Fe}(1)$ & $C(26)$ & $40.4(3)$ \\
\hline$C(25)$ & $\mathrm{Fe}(1)$ & $\mathrm{C}(27)$ & $68.6(3)$ & $\mathrm{C}(25)$ & $\mathrm{Fe}(1)$ & $\mathrm{C}(28)$ & $68.0(3)$ \\
\hline$C(26)$ & $\mathrm{Fe}(1)$ & $\mathrm{C}(27)$ & $40.8(3)$ & $C(26)$ & $\mathrm{Fe}(1)$ & $\mathrm{C}(28)$ & $67.9(3)$ \\
\hline $\mathrm{C}(27)$ & $\mathrm{Fe}(1)$ & $\mathrm{C}(28)$ & $40.6(3)$ & $\operatorname{Pd}(1)$ & $\mathrm{P}(1)$ & $\mathrm{C}(7)$ & $115.7(2)$ \\
\hline $\operatorname{Pd}(1)$ & $\mathrm{P}(1)$ & $\mathrm{C}(13)$ & $114.5(2)$ & $\operatorname{Pd}(1)$ & $\mathrm{P}(1)$ & $\mathrm{C}(19)$ & $110.6(2)$ \\
\hline$C(7)$ & $\mathrm{P}(1)$ & $\mathrm{C}(13)$ & $104.9(3)$ & $\mathrm{C}(7)$ & $\mathrm{P}(1)$ & $\mathrm{C}(19)$ & $97.3(3)$ \\
\hline$C(13)$ & $\mathrm{P}(1)$ & $\mathrm{C}(19)$ & $112.5(3)$ & $\operatorname{Pd}(1)$ & $\mathrm{P}(2)$ & $\mathrm{C}(29)$ & $108.4(2)$ \\
\hline $\operatorname{Pd}(1)$ & $\mathrm{P}(2)$ & $\mathrm{C}(31)$ & $109.7(2)$ & $\operatorname{Pd}(1)$ & $\mathrm{P}(2)$ & $C(35)$ & $114.7(2)$ \\
\hline C(29) & $\mathrm{P}(2)$ & $\mathrm{C}(31)$ & $102.0(3)$ & $\mathrm{C}(29)$ & $\mathrm{P}(2)$ & $\mathrm{C}(35)$ & $109.6(3)$ \\
\hline
\end{tabular}


Table S10. Bond Angles $\left({ }^{\circ}\right)$ for 5 (continued)

\begin{tabular}{|c|c|c|c|c|c|c|c|}
\hline atom & atom & atom & angle & atom & atom & atom & angle \\
\hline $\mathrm{C}(31)$ & $\mathrm{P}(2)$ & $C(35)$ & $111.6(3)$ & $\mathrm{C}(40)$ & $\mathrm{O}(1)$ & $\mathrm{C}(43)$ & 111.7(10) \\
\hline $\operatorname{Pd}(1)$ & $\mathrm{C}(1)$ & $\mathrm{C}(2)$ & $129.8(5)$ & $\operatorname{Pd}(1)$ & $\mathrm{C}(1)$ & $C(6)$ & $112.4(4)$ \\
\hline $\mathrm{C}(2)$ & $\mathrm{C}(1)$ & $C(6)$ & $117.7(6)$ & $\mathrm{C}(1)$ & $C(2)$ & $\mathrm{C}(3)$ & $120.6(7)$ \\
\hline$C(2)$ & $\mathrm{C}(3)$ & $\mathrm{C}(4)$ & $120.5(6)$ & $\mathrm{C}(3)$ & $\mathrm{C}(4)$ & $C(5)$ & 119.1(7) \\
\hline $\mathrm{C}(4)$ & $C(5)$ & $C(6)$ & $118.8(7)$ & $\mathrm{C}(1)$ & $C(6)$ & $C(5)$ & $123.1(6)$ \\
\hline $\mathrm{P}(1)$ & $C(7)$ & $\mathrm{C}(8)$ & $117.8(4)$ & $\mathrm{P}(1)$ & $C(7)$ & $\mathrm{C}(12)$ & $120.9(4)$ \\
\hline $\mathrm{C}(8)$ & $C(7)$ & $\mathrm{C}(12)$ & $120.4(5)$ & $C(7)$ & $\mathrm{C}(8)$ & $\mathrm{C}(9)$ & $119.2(6)$ \\
\hline $\mathrm{C}(8)$ & $\mathrm{C}(9)$ & $\mathrm{C}(10)$ & $120.3(6)$ & $\mathrm{C}(9)$ & $C(10)$ & $\mathrm{C}(11)$ & $120.1(6)$ \\
\hline$C(10)$ & $\mathrm{C}(11)$ & $\mathrm{C}(12)$ & $120.6(6)$ & $C(7)$ & $\mathrm{C}(12)$ & $\mathrm{C}(11)$ & $119.3(5)$ \\
\hline $\mathrm{P}(1)$ & $C(13)$ & $\mathrm{C}(14)$ & 119.1(4) & $\mathrm{P}(1)$ & $\mathrm{C}(13)$ & $\mathrm{C}(18)$ & $121.3(5)$ \\
\hline$C(14)$ & $\mathrm{C}(13)$ & $\mathrm{C}(18)$ & 119.2(6) & $\mathrm{C}(13)$ & $\mathrm{C}(14)$ & $\mathrm{C}(15)$ & $120.0(6)$ \\
\hline$C(14)$ & $\mathrm{C}(15)$ & $C(16)$ & $120.5(6)$ & $C(15)$ & $C(16)$ & $\mathrm{C}(17)$ & $120.3(6)$ \\
\hline$C(16)$ & $\mathrm{C}(17)$ & $\mathrm{C}(18)$ & $120.4(6)$ & $\mathrm{C}(13)$ & $\mathrm{C}(18)$ & $\mathrm{C}(17)$ & $119.4(6)$ \\
\hline $\mathrm{Fe}(1)$ & $\mathrm{C}(19)$ & $\mathrm{P}(1)$ & $137.3(3)$ & $\mathrm{Fe}(1)$ & $\mathrm{C}(19)$ & $\mathrm{C}(20)$ & $68.8(3)$ \\
\hline $\mathrm{Fe}(1)$ & $\mathrm{C}(19)$ & $\mathrm{C}(23)$ & $71.1(3)$ & $\mathrm{P}(1)$ & $\mathrm{C}(19)$ & $\mathrm{C}(20)$ & $125.4(5)$ \\
\hline $\mathrm{P}(1)$ & $C(19)$ & $\mathrm{C}(23)$ & $125.6(4)$ & $\mathrm{C}(20)$ & $\mathrm{C}(19)$ & $\mathrm{C}(23)$ & $107.2(5)$ \\
\hline $\mathrm{Fe}(1)$ & $C(20)$ & $\mathrm{C}(19)$ & $70.1(3)$ & $\mathrm{Fe}(1)$ & $C(20)$ & $\mathrm{C}(21)$ & $70.4(3)$ \\
\hline C(19) & $\mathrm{C}(20)$ & $\mathrm{C}(21)$ & $108.6(5)$ & $\mathrm{Fe}(1)$ & $\mathrm{C}(21)$ & $\mathrm{C}(20)$ & $69.2(4)$ \\
\hline $\mathrm{Fe}(1)$ & $\mathrm{C}(21)$ & $\mathrm{C}(22)$ & $70.1(4)$ & $C(20)$ & $\mathrm{C}(21)$ & $\mathrm{C}(22)$ & $108.5(6)$ \\
\hline $\mathrm{Fe}(1)$ & $\mathrm{C}(22)$ & $\mathrm{C}(21)$ & $69.5(4)$ & $\mathrm{Fe}(1)$ & $\mathrm{C}(22)$ & $\mathrm{C}(23)$ & 71.1(4) \\
\hline $\mathrm{C}(21)$ & $\mathrm{C}(22)$ & $\mathrm{C}(23)$ & $108.4(6)$ & $\mathrm{Fe}(1)$ & $\mathrm{C}(23)$ & $\mathrm{C}(19)$ & $68.0(3)$ \\
\hline $\mathrm{Fe}(1)$ & $\mathrm{C}(23)$ & $\mathrm{C}(22)$ & $68.5(3)$ & $\mathrm{Fe}(1)$ & $\mathrm{C}(23)$ & $\mathrm{C}(29)$ & $131.3(4)$ \\
\hline$C(19)$ & $\mathrm{C}(23)$ & $\mathrm{C}(22)$ & $107.3(5)$ & $C(19)$ & $\mathrm{C}(23)$ & $\mathrm{C}(29)$ & $124.1(6)$ \\
\hline$C(22)$ & $\mathrm{C}(23)$ & $\mathrm{C}(29)$ & $128.5(6)$ & $\mathrm{Fe}(1)$ & $\mathrm{C}(24)$ & $C(25)$ & $69.4(4)$ \\
\hline $\mathrm{Fe}(1)$ & $\mathrm{C}(24)$ & $\mathrm{C}(28)$ & $70.2(4)$ & $\mathrm{C}(25)$ & $\mathrm{C}(24)$ & $\mathrm{C}(28)$ & $107.7(7)$ \\
\hline $\mathrm{Fe}(1)$ & $C(25)$ & $\mathrm{C}(24)$ & $69.8(4)$ & $\mathrm{Fe}(1)$ & $\mathrm{C}(25)$ & $C(26)$ & $70.3(4)$ \\
\hline $\mathrm{C}(24)$ & $C(25)$ & $C(26)$ & 108.2(7) & $\mathrm{Fe}(1)$ & $C(26)$ & $\mathrm{C}(25)$ & $69.3(4)$ \\
\hline $\mathrm{Fe}(1)$ & $\mathrm{C}(26)$ & $\mathrm{C}(27)$ & $69.5(4)$ & $\mathrm{C}(25)$ & $C(26)$ & $\mathrm{C}(27)$ & $108.4(6)$ \\
\hline $\mathrm{Fe}(1)$ & $\mathrm{C}(27)$ & $C(26)$ & $69.7(4)$ & $\mathrm{Fe}(1)$ & $\mathrm{C}(27)$ & $\mathrm{C}(28)$ & $69.8(4)$ \\
\hline$C(26)$ & $\mathrm{C}(27)$ & $\mathrm{C}(28)$ & $106.9(7)$ & $\mathrm{Fe}(1)$ & $\mathrm{C}(28)$ & $\mathrm{C}(24)$ & $69.4(4)$ \\
\hline $\mathrm{Fe}(1)$ & $C(28)$ & $\mathrm{C}(27)$ & $69.6(4)$ & $C(24)$ & $\mathrm{C}(28)$ & $C(27)$ & $108.8(7)$ \\
\hline
\end{tabular}


Table S10. Bond Angles $\left({ }^{\circ}\right)$ for 5 (continued)

$\begin{array}{llllllll}\text { atom } & \text { atom } & \text { atom } & \text { angle } & \text { atom } & \text { atom } & \text { atom } & \text { angle } \\ \mathrm{P}(2) & \mathrm{C}(29) & \mathrm{C}(23) & 108.8(4) & \mathrm{P}(2) & \mathrm{C}(29) & \mathrm{C}(30) & 122.1(4) \\ \mathrm{C}(23) & \mathrm{C}(29) & \mathrm{C}(30) & 111.0(6) & \mathrm{P}(2) & \mathrm{C}(31) & \mathrm{C}(32) & 110.9(4) \\ \mathrm{P}(2) & \mathrm{C}(31) & \mathrm{C}(33) & 107.0(4) & \mathrm{P}(2) & \mathrm{C}(31) & \mathrm{C}(34) & 115.7(5) \\ \mathrm{C}(32) & \mathrm{C}(31) & \mathrm{C}(33) & 105.6(6) & \mathrm{C}(32) & \mathrm{C}(31) & \mathrm{C}(34) & 107.6(5) \\ \mathrm{C}(33) & \mathrm{C}(31) & \mathrm{C}(34) & 109.4(5) & \mathrm{P}(2) & \mathrm{C}(35) & \mathrm{C}(36) & 105.7(4) \\ \mathrm{P}(2) & \mathrm{C}(35) & \mathrm{C}(37) & 115.1(4) & \mathrm{P}(2) & \mathrm{C}(35) & \mathrm{C}(38) & 111.8(5) \\ \mathrm{C}(36) & \mathrm{C}(35) & \mathrm{C}(37) & 108.1(6) & \mathrm{C}(36) & \mathrm{C}(35) & \mathrm{C}(38) & 107.0(5) \\ \mathrm{C}(37) & \mathrm{C}(35) & \mathrm{C}(38) & 108.7(5) & \mathrm{O}(1) & \mathrm{C}(40) & \mathrm{C}(41) & 105(1) \\ \mathrm{C}(40) & \mathrm{C}(41) & \mathrm{C}(42) & 100(1) & \mathrm{C}(41) & \mathrm{C}(42) & \mathrm{C}(43) & 105(1) \\ \mathrm{O}(1) & \mathrm{C}(43) & \mathrm{C}(42) & 108(1) & & & & \end{array}$


Table S11. Bond Angles for the Hydrogen Atoms $\left({ }^{\circ}\right)$ for 5

\begin{tabular}{|c|c|c|c|c|c|c|c|}
\hline atom & atom & atom & angle & atom & atom & atom & angle \\
\hline $\mathrm{C}(1)$ & $\mathrm{C}(2)$ & $\mathrm{H}(1)$ & 119.7 & $\mathrm{C}(3)$ & $\mathrm{C}(2)$ & $\mathrm{H}(1)$ & 119.7 \\
\hline $\mathrm{C}(2)$ & $\mathrm{C}(3)$ & $\mathrm{H}(2)$ & 119.7 & $\mathrm{C}(4)$ & $\mathrm{C}(3)$ & $\mathrm{H}(2)$ & 119.7 \\
\hline $\mathrm{C}(3)$ & $\mathrm{C}(4)$ & $\mathrm{H}(3)$ & 120.4 & $C(5)$ & $\mathrm{C}(4)$ & $\mathrm{H}(3)$ & 120.4 \\
\hline $\mathrm{C}(4)$ & $\mathrm{C}(5)$ & $\mathrm{H}(4)$ & 120.6 & $\mathrm{C}(6)$ & $\mathrm{C}(5)$ & $\mathrm{H}(4)$ & 120.6 \\
\hline $\mathrm{C}(1)$ & $\mathrm{C}(6)$ & $\mathrm{H}(5)$ & 118.4 & $C(5)$ & $\mathrm{C}(6)$ & $\mathrm{H}(5)$ & 118.4 \\
\hline$C(7)$ & $\mathrm{C}(8)$ & $\mathrm{H}(6)$ & 120.4 & $\mathrm{C}(9)$ & $\mathrm{C}(8)$ & $\mathrm{H}(6)$ & 120.4 \\
\hline $\mathrm{C}(8)$ & $\mathrm{C}(9)$ & $\mathrm{H}(7)$ & 119.9 & $C(10)$ & $\mathrm{C}(9)$ & $\mathrm{H}(7)$ & 119.9 \\
\hline $\mathrm{C}(9)$ & $C(10)$ & $\mathrm{H}(8)$ & 119.9 & $\mathrm{C}(11)$ & $C(10)$ & $\mathrm{H}(8)$ & 119.9 \\
\hline $\mathrm{C}(10)$ & $\mathrm{C}(11)$ & $\mathrm{H}(9)$ & 119.7 & $\mathrm{C}(12)$ & $\mathrm{C}(11)$ & $\mathrm{H}(9)$ & 119.7 \\
\hline $\mathrm{C}(7)$ & $\mathrm{C}(12)$ & $\mathrm{H}(10)$ & 120.3 & $\mathrm{C}(11)$ & $\mathrm{C}(12)$ & $\mathrm{H}(10)$ & 120.3 \\
\hline $\mathrm{C}(13)$ & $\mathrm{C}(14)$ & $\mathrm{H}(11)$ & 120.0 & $C(15)$ & $\mathrm{C}(14)$ & $\mathrm{H}(11)$ & 120.0 \\
\hline $\mathrm{C}(14)$ & $C(15)$ & $\mathrm{H}(12)$ & 119.7 & $C(16)$ & $\mathrm{C}(15)$ & $\mathrm{H}(12)$ & 119.8 \\
\hline $\mathrm{C}(15)$ & $C(16)$ & $\mathrm{H}(13)$ & 119.8 & $\mathrm{C}(17)$ & $C(16)$ & $\mathrm{H}(13)$ & 119.8 \\
\hline $\mathrm{C}(16)$ & $C(17)$ & $\mathrm{H}(14)$ & 119.8 & $\mathrm{C}(18)$ & $\mathrm{C}(17)$ & $\mathrm{H}(14)$ & 119.8 \\
\hline $\mathrm{C}(13)$ & $\mathrm{C}(18)$ & $\mathrm{H}(15)$ & 120.3 & $\mathrm{C}(17)$ & $\mathrm{C}(18)$ & $\mathrm{H}(15)$ & 120.3 \\
\hline $\mathrm{Fe}(1)$ & $\mathrm{C}(20)$ & $\mathrm{H}(16)$ & 125.4 & $\mathrm{C}(19)$ & $\mathrm{C}(20)$ & $\mathrm{H}(16)$ & 125.7 \\
\hline $\mathrm{C}(21)$ & $\mathrm{C}(20)$ & $\mathrm{H}(16)$ & 125.7 & $\mathrm{Fe}(1)$ & $\mathrm{C}(21)$ & $\mathrm{H}(17)$ & 126.5 \\
\hline $\mathrm{C}(20)$ & $\mathrm{C}(21)$ & $\mathrm{H}(17)$ & 125.8 & $\mathrm{C}(22)$ & $\mathrm{C}(21)$ & $\mathrm{H}(17)$ & 125.8 \\
\hline $\mathrm{Fe}(1)$ & $C(22)$ & $\mathrm{H}(18)$ & 125.2 & $C(21)$ & $\mathrm{C}(22)$ & $\mathrm{H}(18)$ & 125.8 \\
\hline $\mathrm{C}(23)$ & $\mathrm{C}(22)$ & $\mathrm{H}(18)$ & 125.8 & $\mathrm{Fe}(1)$ & $\mathrm{C}(24)$ & $\mathrm{H}(19)$ & 125.8 \\
\hline $\mathrm{C}(25)$ & $C(24)$ & $\mathrm{H}(19)$ & 126.1 & $\mathrm{C}(28)$ & $\mathrm{C}(24)$ & $\mathrm{H}(19)$ & 126.1 \\
\hline $\mathrm{Fe}(1)$ & $C(25)$ & $\mathrm{H}(20)$ & 125.5 & $\mathrm{C}(24)$ & $\mathrm{C}(25)$ & $\mathrm{H}(20)$ & 125.9 \\
\hline$C(26)$ & $C(25)$ & $\mathrm{H}(20)$ & 125.9 & $\mathrm{Fe}(1)$ & $\mathrm{C}(26)$ & $\mathrm{H}(21)$ & 127.0 \\
\hline $\mathrm{C}(25)$ & $C(26)$ & $\mathrm{H}(21)$ & 125.8 & $C(27)$ & $C(26)$ & $\mathrm{H}(21)$ & 125.8 \\
\hline $\mathrm{Fe}(1)$ & $\mathrm{C}(27)$ & $\mathrm{H}(22)$ & 125.5 & $C(26)$ & $\mathrm{C}(27)$ & $\mathrm{H}(22)$ & 126.5 \\
\hline $\mathrm{C}(28)$ & $\mathrm{C}(27)$ & $\mathrm{H}(22)$ & 126.5 & $\mathrm{Fe}(1)$ & $\mathrm{C}(28)$ & $\mathrm{H}(23)$ & 127.0 \\
\hline $\mathrm{C}(24)$ & $\mathrm{C}(28)$ & $\mathrm{H}(23)$ & 125.6 & $\mathrm{C}(27)$ & $\mathrm{C}(28)$ & $\mathrm{H}(23)$ & 125.6 \\
\hline $\mathrm{P}(2)$ & $\mathrm{C}(29)$ & $\mathrm{H}(24)$ & 104.4 & $\mathrm{C}(23)$ & $\mathrm{C}(29)$ & $\mathrm{H}(24)$ & 104.4 \\
\hline $\mathrm{C}(30)$ & $\mathrm{C}(29)$ & $\mathrm{H}(24)$ & 104.4 & $\mathrm{C}(29)$ & $\mathrm{C}(30)$ & $\mathrm{H}(25)$ & 109.5 \\
\hline $\mathrm{C}(29)$ & $\mathrm{C}(30)$ & $\mathrm{H}(26)$ & 109.5 & $C(29)$ & $\mathrm{C}(30)$ & $\mathrm{H}(27)$ & 109.5 \\
\hline $\mathrm{H}(25)$ & $\mathrm{C}(30)$ & $\mathrm{H}(26)$ & 109.5 & $\mathrm{H}(25)$ & $\mathrm{C}(30)$ & $\mathrm{H}(27)$ & 109.5 \\
\hline
\end{tabular}


Table S11. Bond Angles for the Hydrogen Atoms $\left({ }^{\circ}\right)$ for 5 (continued)

\begin{tabular}{|c|c|c|c|c|c|c|c|}
\hline atom & atom & atom & angle & atom & atom & atom & angle \\
\hline $\mathrm{H}(26)$ & $\mathrm{C}(30)$ & $\mathrm{H}(27)$ & 109.5 & $\mathrm{C}(31)$ & $\mathrm{C}(32)$ & $\mathrm{H}(28)$ & 109.5 \\
\hline$C(31)$ & $\mathrm{C}(32)$ & $\mathrm{H}(29)$ & 109.5 & $\mathrm{C}(31)$ & $\mathrm{C}(32)$ & $\mathrm{H}(30)$ & 109.5 \\
\hline $\mathrm{H}(28)$ & $\mathrm{C}(32)$ & $\mathrm{H}(29)$ & 109.5 & $\mathrm{H}(28)$ & $\mathrm{C}(32)$ & $\mathrm{H}(30)$ & 109.5 \\
\hline $\mathrm{H}(29)$ & $\mathrm{C}(32)$ & $\mathrm{H}(30)$ & 109.5 & $\mathrm{C}(31)$ & $\mathrm{C}(33)$ & $\mathrm{H}(31)$ & 109.5 \\
\hline $\mathrm{C}(31)$ & $\mathrm{C}(33)$ & $\mathrm{H}(32)$ & 109.5 & $\mathrm{C}(31)$ & $\mathrm{C}(33)$ & $\mathrm{H}(33)$ & 109.5 \\
\hline $\mathrm{H}(31)$ & $\mathrm{C}(33)$ & $\mathrm{H}(32)$ & 109.5 & $\mathrm{H}(31)$ & $\mathrm{C}(33)$ & $\mathrm{H}(33)$ & 109.5 \\
\hline $\mathrm{H}(32)$ & $\mathrm{C}(33)$ & $\mathrm{H}(33)$ & 109.5 & $\mathrm{C}(31)$ & $\mathrm{C}(34)$ & $\mathrm{H}(34)$ & 109.5 \\
\hline $\mathrm{C}(31)$ & $\mathrm{C}(34)$ & $\mathrm{H}(35)$ & 109.5 & $\mathrm{C}(31)$ & $C(34)$ & $\mathrm{H}(36)$ & 109.5 \\
\hline $\mathrm{H}(34)$ & $C(34)$ & $\mathrm{H}(35)$ & 109.5 & $\mathrm{H}(34)$ & $\mathrm{C}(34)$ & $\mathrm{H}(36)$ & 109.5 \\
\hline $\mathrm{H}(35)$ & $C(34)$ & $\mathrm{H}(36)$ & 109.5 & $C(35)$ & $C(36)$ & $\mathrm{H}(37)$ & 109.5 \\
\hline$C(35)$ & $C(36)$ & $\mathrm{H}(38)$ & 109.5 & $C(35)$ & $\mathrm{C}(36)$ & $\mathrm{H}(39)$ & 109.5 \\
\hline $\mathrm{H}(37)$ & $C(36)$ & $\mathrm{H}(38)$ & 109.5 & $\mathrm{H}(37)$ & $\mathrm{C}(36)$ & $\mathrm{H}(39)$ & 109.5 \\
\hline $\mathrm{H}(38)$ & $\mathrm{C}(36)$ & $\mathrm{H}(39)$ & 109.5 & $C(35)$ & $\mathrm{C}(37)$ & $\mathrm{H}(40)$ & 109.5 \\
\hline$C(35)$ & $\mathrm{C}(37)$ & $\mathrm{H}(41)$ & 109.5 & $C(35)$ & $\mathrm{C}(37)$ & $\mathrm{H}(42)$ & 109.5 \\
\hline $\mathrm{H}(40)$ & $\mathrm{C}(37)$ & $\mathrm{H}(41)$ & 109.5 & $\mathrm{H}(40)$ & $\mathrm{C}(37)$ & $\mathrm{H}(42)$ & 109.5 \\
\hline $\mathrm{H}(41)$ & $\mathrm{C}(37)$ & $\mathrm{H}(42)$ & 109.5 & $C(35)$ & $\mathrm{C}(38)$ & $\mathrm{H}(43)$ & 109.5 \\
\hline$C(35)$ & $C(38)$ & $\mathrm{H}(44)$ & 109.5 & $C(35)$ & $\mathrm{C}(38)$ & $\mathrm{H}(45)$ & 109.5 \\
\hline $\mathrm{H}(43)$ & $C(38)$ & $\mathrm{H}(44)$ & 109.5 & $\mathrm{H}(43)$ & $\mathrm{C}(38)$ & $\mathrm{H}(45)$ & 109.5 \\
\hline $\mathrm{H}(44)$ & $\mathrm{C}(38)$ & $\mathrm{H}(45)$ & 109.5 & $\mathrm{O}(1)$ & $\mathrm{C}(40)$ & $\mathrm{H}(46)$ & 110.5 \\
\hline $\mathrm{O}(1)$ & $\mathrm{C}(40)$ & $\mathrm{H}(47)$ & 110.5 & $\mathrm{C}(41)$ & $\mathrm{C}(40)$ & $\mathrm{H}(46)$ & 110.5 \\
\hline$C(41)$ & $\mathrm{C}(40)$ & $\mathrm{H}(47)$ & 110.5 & $\mathrm{H}(46)$ & $\mathrm{C}(40)$ & $\mathrm{H}(47)$ & 109.5 \\
\hline$C(40)$ & $\mathrm{C}(41)$ & $\mathrm{H}(48)$ & 111.6 & $\mathrm{C}(40)$ & $\mathrm{C}(41)$ & $\mathrm{H}(49)$ & 111.6 \\
\hline$C(42)$ & $\mathrm{C}(41)$ & $\mathrm{H}(48)$ & 111.6 & $C(42)$ & $\mathrm{C}(41)$ & $\mathrm{H}(49)$ & 111.6 \\
\hline $\mathrm{H}(48)$ & $\mathrm{C}(41)$ & $\mathrm{H}(49)$ & 109.5 & $C(41)$ & $C(42)$ & $\mathrm{H}(50)$ & 110.4 \\
\hline$C(41)$ & $\mathrm{C}(42)$ & $\mathrm{H}(51)$ & 110.4 & $\mathrm{C}(43)$ & $\mathrm{C}(42)$ & $\mathrm{H}(50)$ & 110.4 \\
\hline $\mathrm{C}(43)$ & $\mathrm{C}(42)$ & $\mathrm{H}(51)$ & 110.4 & $\mathrm{H}(50)$ & $\mathrm{C}(42)$ & $\mathrm{H}(51)$ & 109.5 \\
\hline $\mathrm{O}(1)$ & $\mathrm{C}(43)$ & $\mathrm{H}(52)$ & 109.6 & $\mathrm{O}(1)$ & $\mathrm{C}(43)$ & $\mathrm{H}(53)$ & 109.7 \\
\hline$C(42)$ & $C(43)$ & $\mathrm{H}(52)$ & 109.7 & $C(42)$ & $\mathrm{C}(43)$ & $\mathrm{H}(53)$ & 109.7 \\
\hline $\mathrm{H}(52)$ & $\mathrm{C}(43)$ & $\mathrm{H}(53)$ & 109.5 & & & & \\
\hline
\end{tabular}


Table S12. Torsion Angles $\left({ }^{\circ}\right)$ for $\mathbf{5}$

atom atom atom atom angle

$\begin{array}{lllll}\mathrm{Pd}(1) & \mathrm{P}(1) & \mathrm{C}(7) & \mathrm{C}(8) & -35.6(6)\end{array}$

$\begin{array}{lllll}\mathrm{Pd}(1) & \mathrm{P}(1) & \mathrm{C}(13) & \mathrm{C}(14) & -83.2(5)\end{array}$

$\begin{array}{lllll}\mathrm{Pd}(1) & \mathrm{P}(1) & \mathrm{C}(19) & \mathrm{Fe}(1) & -144.2(4)\end{array}$

$\begin{array}{lllll}\mathrm{Pd}(1) & \mathrm{P}(1) & \mathrm{C}(19) & \mathrm{C}(23) & -43.5(5)\end{array}$

$\begin{array}{lllll}\mathrm{Pd}(1) & \mathrm{P}(2) & \mathrm{C}(29) & \mathrm{C}(30) & 167.6(5)\end{array}$

$\begin{array}{lllll}\mathrm{Pd}(1) & \mathrm{P}(2) & \mathrm{C}(31) & \mathrm{C}(33) & -67.4(5)\end{array}$

$\begin{array}{lllll}\mathrm{Pd}(1) & \mathrm{P}(2) & \mathrm{C}(35) & \mathrm{C}(36) & 35.9(5)\end{array}$

$\begin{array}{lllll}\mathrm{Pd}(1) & \mathrm{P}(2) & \mathrm{C}(35) & \mathrm{C}(38) & -80.3(5)\end{array}$

$\begin{array}{lllll}\mathrm{Pd}(1) & \mathrm{C}(1) & \mathrm{C}(6) & \mathrm{C}(5) & -178.6(5)\end{array}$

$\operatorname{Br}(1) \quad \operatorname{Pd}(1) \quad \mathrm{P}(1) \quad \mathrm{C}(13) \quad 125.1(2)$

$\operatorname{Br}(1) \quad \operatorname{Pd}(1) \quad \mathrm{P}(2) \quad \mathrm{C}(29) \quad 177.9(2)$

$\operatorname{Br}(1) \quad \operatorname{Pd}(1) \quad \mathrm{P}(2) \quad \mathrm{C}(35) \quad 55.1(2)$

$\operatorname{Br}(1) \quad \operatorname{Pd}(1) \quad \mathrm{C}(1) \quad \mathrm{C}(6) \quad 94.9(4)$

$\begin{array}{lllll}\mathrm{Fe}(1) & \mathrm{C}(19) & \mathrm{P}(1) & \mathrm{C}(13) & -14.8(5)\end{array}$

$\begin{array}{lllll}\mathrm{Fe}(1) & \mathrm{C}(19) & \mathrm{C}(23) & \mathrm{C}(22) & -57.4(4)\end{array}$

$\mathrm{Fe}(1) \quad \mathrm{C}(20) \quad \mathrm{C}(19) \quad \mathrm{P}(1) \quad 133.7(4)$

$\begin{array}{lllll}\mathrm{Fe}(1) & \mathrm{C}(20) & \mathrm{C}(21) & \mathrm{C}(22) & 59.3(4)\end{array}$

$\begin{array}{lllll}\mathrm{Fe}(1) & \mathrm{C}(21) & \mathrm{C}(22) & \mathrm{C}(23) & 60.7(4)\end{array}$

$\mathrm{Fe}(1) \quad \mathrm{C}(22) \quad \mathrm{C}(23) \quad \mathrm{C}(19) \quad 57.1(4)$

$\begin{array}{lllll}\mathrm{Fe}(1) & \mathrm{C}(23) & \mathrm{C}(19) & \mathrm{P}(1) & -135.2(4)\end{array}$

$\begin{array}{lllll}\mathrm{Fe}(1) & \mathrm{C}(23) & \mathrm{C}(22) & \mathrm{C}(21) & -59.7(4)\end{array}$

$\begin{array}{lllll}\mathrm{Fe}(1) & \mathrm{C}(23) & \mathrm{C}(29) & \mathrm{C}(30) & -65.4(7)\end{array}$

$\begin{array}{lllll}\mathrm{Fe}(1) & \mathrm{C}(24) & \mathrm{C}(28) & \mathrm{C}(27) & 58.6(5)\end{array}$

$\begin{array}{lllll}\mathrm{Fe}(1) & \mathrm{C}(25) & \mathrm{C}(26) & \mathrm{C}(27) & -58.7(5)\end{array}$

$\begin{array}{lllll}\mathrm{Fe}(1) & \mathrm{C}(26) & \mathrm{C}(27) & \mathrm{C}(28) & -60.2(4)\end{array}$

$\begin{array}{lllll}\mathrm{Fe}(1) & \mathrm{C}(27) & \mathrm{C}(28) & \mathrm{C}(24) & -58.5(5)\end{array}$

$\begin{array}{lllll}\mathrm{Fe}(1) & \mathrm{C}(28) & \mathrm{C}(27) & \mathrm{C}(26) & 60.1(5)\end{array}$

$\mathrm{P}(1) \quad \mathrm{Pd}(1) \quad \mathrm{P}(2) \quad \mathrm{C}(31) \quad 124.0(2)$

$\begin{array}{lllll}\mathrm{P}(1) & \mathrm{Pd}(1) & \mathrm{C}(1) & \mathrm{C}(2) & 77.3(5)\end{array}$

$\begin{array}{lllll}\mathrm{P}(1) & \mathrm{C}(7) & \mathrm{C}(8) & \mathrm{C}(9) & -169.6(5)\end{array}$

$\mathrm{P}(1) \quad \mathrm{C}(13) \quad \mathrm{C}(14) \quad \mathrm{C}(15) \quad 176.8(5)$ atom atom atom atom angle

$\begin{array}{lllll}\mathrm{Pd}(1) & \mathrm{P}(1) & \mathrm{C}(7) & \mathrm{C}(12) & 155.7(5)\end{array}$

$\begin{array}{lllll}\mathrm{Pd}(1) & \mathrm{P}(1) & \mathrm{C}(13) & \mathrm{C}(18) & 89.4(5)\end{array}$

$\begin{array}{lllll}\mathrm{Pd}(1) & \mathrm{P}(1) & \mathrm{C}(19) & \mathrm{C}(20) & 119.1(5)\end{array}$

$\begin{array}{lllll}\mathrm{Pd}(1) & \mathrm{P}(2) & \mathrm{C}(29) & \mathrm{C}(23) & -61.1(5)\end{array}$

$\begin{array}{lllll}\mathrm{Pd}(1) & \mathrm{P}(2) & \mathrm{C}(31) & \mathrm{C}(32) & 47.3(5)\end{array}$

$\begin{array}{lllll}\mathrm{Pd}(1) & \mathrm{P}(2) & \mathrm{C}(31) & \mathrm{C}(34) & 170.3(4)\end{array}$

$\begin{array}{lllll}\mathrm{Pd}(1) & \mathrm{P}(2) & \mathrm{C}(35) & \mathrm{C}(37) & 155.1(4)\end{array}$

$\begin{array}{lllll}\mathrm{Pd}(1) & \mathrm{C}(1) & \mathrm{C}(2) & \mathrm{C}(3) & 179.0(4)\end{array}$

$\begin{array}{llll}\mathrm{Br}(1) & \mathrm{Pd}(1) & \mathrm{P}(1) & \mathrm{C}(7)\end{array}$

$\begin{array}{lllll}\mathrm{Br}(1) & \mathrm{Pd}(1) & \mathrm{P}(1) & \mathrm{C}(19) & -106.5(2)\end{array}$

$\begin{array}{lllll}\operatorname{Br}(1) & \operatorname{Pd}(1) & P(2) & C(31) & -71.5(2)\end{array}$

$\mathrm{Br}(1) \quad \mathrm{Pd}(1) \quad \mathrm{C}(1) \quad \mathrm{C}(2) \quad-83.0(5)$

$\begin{array}{lllll}\mathrm{Fe}(1) & \mathrm{C}(19) & \mathrm{P}(1) & \mathrm{C}(7) & 94.8(5)\end{array}$

$\mathrm{Fe}(1) \quad \mathrm{C}(19) \quad \mathrm{C}(20) \quad \mathrm{C}(21) \quad 60.1(4)$

$\mathrm{Fe}(1) \quad \mathrm{C}(19) \quad \mathrm{C}(23) \quad \mathrm{C}(29) \quad 125.9(5)$

$\mathrm{Fe}(1) \quad \mathrm{C}(20) \quad \mathrm{C}(19) \quad \mathrm{C}(23) \quad-61.0(4)$

$\mathrm{Fe}(1) \quad \mathrm{C}(21) \quad \mathrm{C}(20) \quad \mathrm{C}(19) \quad-59.9(4)$

$\mathrm{Fe}(1) \quad \mathrm{C}(22) \quad \mathrm{C}(21) \quad \mathrm{C}(20) \quad-58.7(4)$

$\mathrm{Fe}(1) \quad \mathrm{C}(22) \quad \mathrm{C}(23) \quad \mathrm{C}(29) \quad-126.4(6)$

$\begin{array}{lllll}\mathrm{Fe}(1) & \mathrm{C}(23) & \mathrm{C}(19) & \mathrm{C}(20) & 59.6(4)\end{array}$

$\begin{array}{lllll}\mathrm{Fe}(1) & \mathrm{C}(23) & \mathrm{C}(29) & \mathrm{P}(2) & 157.6(4)\end{array}$

$\begin{array}{lllll}\mathrm{Fe}(1) & \mathrm{C}(24) & \mathrm{C}(25) & \mathrm{C}(26) & -60.1(5)\end{array}$

$\mathrm{Fe}(1) \quad \mathrm{C}(25) \quad \mathrm{C}(24) \quad \mathrm{C}(28) \quad 60.0(5)$

$\begin{array}{lllll}\mathrm{Fe}(1) & \mathrm{C}(26) & \mathrm{C}(25) & \mathrm{C}(24) & 59.8(4)\end{array}$

$\begin{array}{lllll}\mathrm{Fe}(1) & \mathrm{C}(27) & \mathrm{C}(26) & \mathrm{C}(25) & 58.5(5)\end{array}$

$\begin{array}{lllll}\mathrm{Fe}(1) & \mathrm{C}(28) & \mathrm{C}(24) & \mathrm{C}(25) & -59.5(4)\end{array}$

$\mathrm{P}(1) \quad \mathrm{Pd}(1) \quad \mathrm{P}(2) \quad \mathrm{C}(29) \quad 13.4(2)$

$\mathrm{P}(1) \quad \mathrm{Pd}(1) \quad \mathrm{P}(2) \quad \mathrm{C}(35) \quad-109.4(2)$

$\mathrm{P}(1) \quad \mathrm{Pd}(1) \quad \mathrm{C}(1) \quad \mathrm{C}(6) \quad-104.7(4)$

$\mathrm{P}(1) \quad \mathrm{C}(7) \quad \mathrm{C}(12) \quad \mathrm{C}(11) \quad 171.1(6)$

$\begin{array}{lllll}\mathrm{P}(1) & \mathrm{C}(13) & \mathrm{C}(18) & \mathrm{C}(17) & -175.6(5)\end{array}$ 
Table S12. Torsion Angles $\left({ }^{\circ}\right)$ for 5 (continued)

\begin{tabular}{|c|c|c|c|c|c|c|c|c|c|}
\hline atom & atom & atom & atom & angle & atom & atom & atom & atom & angle \\
\hline$P(1)$ & $C(19)$ & $\mathrm{Fe}(1)$ & $C(20)$ & $-119.8(6)$ & $\mathrm{P}(1)$ & $C(19)$ & $\mathrm{Fe}(1)$ & $\mathrm{C}(21)$ & $-157.2(5)$ \\
\hline $\mathrm{P}(1)$ & $C(19)$ & $\mathrm{Fe}(1)$ & $C(22)$ & 159.3(5) & $\mathrm{P}(1)$ & $C(19)$ & $\mathrm{Fe}(1)$ & $C(23)$ & $122.3(6)$ \\
\hline (1) & $\mathrm{C}(19)$ & $\mathrm{Fe}(1)$ & $\mathrm{C}(24)$ & $38.0(6)$ & $\mathrm{P}(1)$ & $C(19)$ & $\mathrm{Fe}(1)$ & $C(25)$ & $-6.2(5)$ \\
\hline $\mathrm{P}(1)$ & $\mathrm{C}(19)$ & $\mathrm{Fe}(1)$ & $C(26)$ & $-49.7(6)$ & $\mathrm{P}(1)$ & $C(19)$ & $\mathrm{Fe}(1)$ & $C(27)$ & $-93.7(10)$ \\
\hline $\mathrm{P}(1)$ & $\mathrm{C}(19)$ & $\mathrm{Fe}(1)$ & $C(28)$ & $79.0(10)$ & $\mathrm{P}(1)$ & $C(19)$ & $C(20)$ & $C(21)$ & $-166.2(4)$ \\
\hline $\mathrm{P}(1)$ & $\mathrm{C}(19)$ & $C(23)$ & $C(22)$ & $167.4(4)$ & $\mathrm{P}(1)$ & $\mathrm{C}(19)$ & $C(23)$ & $\mathrm{C}(29)$ & $-9.3(8)$ \\
\hline $\mathrm{P}(2)$ & $\operatorname{Pd}(1)$ & $\mathrm{P}(1)$ & $C(7)$ & 138.0(2) & $\mathrm{P}(2)$ & $\operatorname{Pd}(1)$ & $\mathrm{P}(1)$ & $\mathrm{C}(13)$ & $-99.7(2)$ \\
\hline $\mathrm{P}(2)$ & $\operatorname{Pd}(1)$ & $\mathrm{P}(1)$ & $C(19)$ & $28.7(2)$ & $\mathrm{P}(2)$ & $\operatorname{Pd}(1)$ & $C(1)$ & $C(2)$ & 170.1(3) \\
\hline $\mathrm{P}(2)$ & $\operatorname{Pd}(1)$ & $\mathrm{C}(1)$ & $C(6)$ & $-12.0(9)$ & $\mathrm{P}(2)$ & $C(29)$ & $C(23)$ & $C(19)$ & $67.9(6)$ \\
\hline $\mathrm{P}(2)$ & $C(29)$ & $\mathrm{C}(23)$ & $C(22)$ & $-108.1(6)$ & $\mathrm{O}(1)$ & $\mathrm{C}(40)$ & $\mathrm{C}(41)$ & $\mathrm{C}(42)$ & $-23(1)$ \\
\hline $\mathrm{O}(1)$ & $\mathrm{C}(43)$ & $\mathrm{C}(42)$ & $\mathrm{C}(41)$ & $-22(1)$ & $C(1)$ & $\operatorname{Pd}(1)$ & $\mathrm{P}(1)$ & $C(7)$ & $-60.6(3)$ \\
\hline $\mathrm{PC}(1)$ & $\operatorname{Pd}(1)$ & $\mathrm{P}(1)$ & $\mathrm{C}(13)$ & $61.7(3)$ & $\mathrm{C}(1)$ & $\operatorname{Pd}(1)$ & $\mathrm{P}(1)$ & $\mathrm{C}(19)$ & $-169.9(3)$ \\
\hline $\mathrm{C}(1)$ & $\operatorname{Pd}(1)$ & $\mathrm{P}(2)$ & $C(29)$ & $-77.2(6)$ & $C(1)$ & $\operatorname{Pd}(1)$ & $\mathrm{P}(2)$ & $C(31)$ & $33.4(5)$ \\
\hline $\mathrm{C}(1)$ & $\operatorname{Pd}(1)$ & $\mathrm{P}(2)$ & $C(35)$ & $159.9(5)$ & $\mathrm{C}(1)$ & $C(2)$ & $C(3)$ & $\mathrm{C}(4)$ & $-0.4(9)$ \\
\hline $\mathrm{C}(1)$ & $C(6)$ & $C(5)$ & $\mathrm{C}(4)$ & $0(1)$ & $\mathrm{C}(2)$ & $\mathrm{C}(1)$ & $C(6)$ & $\mathrm{C}(5)$ & $-0.5(10)$ \\
\hline $\mathrm{C}(2)$ & $C(3)$ & $\mathrm{C}(4)$ & $C(5)$ & $-1.0(9)$ & $\mathrm{C}(3)$ & $\mathrm{C}(2)$ & $\mathrm{C}(1)$ & $C(6)$ & $1.1(9)$ \\
\hline$C(3)$ & $\mathrm{C}(4)$ & $C(5)$ & $C(6)$ & $1.7(10)$ & $C(7)$ & $\mathrm{P}(1)$ & $C(13)$ & $C(14)$ & $44.8(5)$ \\
\hline $\mathrm{C}(7)$ & $\mathrm{P}(1)$ & $\mathrm{C}(13)$ & $C(18)$ & $-142.7(5)$ & $\mathrm{C}(7)$ & $\mathrm{P}(1)$ & $C(19)$ & $C(20)$ & $-1.9(5)$ \\
\hline $\mathrm{C}(7)$ & $\mathrm{P}(1)$ & $\mathrm{C}(19)$ & $C(23)$ & $-164.5(5)$ & $\mathrm{C}(7)$ & $C(8)$ & $\mathrm{C}(9)$ & $\mathrm{C}(10)$ & $-1(1)$ \\
\hline $\mathrm{C}(7)$ & $\mathrm{C}(12)$ & $\mathrm{C}(11)$ & $C(10)$ & $-2(1)$ & $C(8)$ & $C(7)$ & $\mathrm{P}(1)$ & $\mathrm{C}(13)$ & $-162.8(5)$ \\
\hline $\mathrm{C}(8)$ & $\mathrm{C}(7)$ & $\mathrm{P}(1)$ & $C(19)$ & $81.5(5)$ & $\mathrm{C}(8)$ & $C(7)$ & $\mathrm{C}(12)$ & $\mathrm{C}(11)$ & $2(1)$ \\
\hline $\mathrm{C}(8)$ & $\mathrm{C}(9)$ & $C(10)$ & $C(11)$ & 1(1) & $\mathrm{C}(9)$ & $C(8)$ & $C(7)$ & $C(12)$ & $0(1)$ \\
\hline $\mathrm{C}(9)$ & $\mathrm{C}(10)$ & $\mathrm{C}(11)$ & $C(12)$ & $0(1)$ & $C(12)$ & $C(7)$ & $\mathrm{P}(1)$ & $C(13)$ & $28.5(6)$ \\
\hline $\mathrm{C}(12)$ & $C(7)$ & $\mathrm{P}(1)$ & $\mathrm{C}(19)$ & $-87.3(6)$ & $\mathrm{C}(13)$ & $\mathrm{P}(1)$ & $\mathrm{C}(19)$ & $C(20)$ & $-111.4(5)$ \\
\hline $\mathrm{C}(13)$ & $\mathrm{P}(1)$ & $\mathrm{C}(19)$ & $C(23)$ & $86.0(5)$ & $C(13)$ & $C(14)$ & $C(15)$ & $C(16)$ & $-1.7(10)$ \\
\hline $\mathrm{C}(13)$ & $\mathrm{C}(18)$ & $\mathrm{C}(17)$ & $C(16)$ & $-0.3(9)$ & $C(14)$ & $C(13)$ & $\mathrm{P}(1)$ & $\mathrm{C}(19)$ & 149.4(5) \\
\hline $\mathrm{C}(14)$ & $\mathrm{C}(13)$ & $\mathrm{C}(18)$ & $C(17)$ & $-3.0(9)$ & $C(14)$ & $C(15)$ & $C(16)$ & $C(17)$ & $-1(1)$ \\
\hline $\mathrm{C}(15)$ & $\mathrm{C}(14)$ & $\mathrm{C}(13)$ & $\mathrm{C}(18)$ & $4.0(9)$ & $\mathrm{C}(15)$ & $C(16)$ & $\mathrm{C}(17)$ & $\mathrm{C}(18)$ & $2(1)$ \\
\hline $\mathrm{C}(18)$ & $\mathrm{C}(13)$ & $\mathrm{P}(1)$ & $\mathrm{C}(19)$ & $-38.0(6)$ & $\mathrm{C}(19)$ & $\mathrm{Fe}(1)$ & $\mathrm{C}(20)$ & $\mathrm{C}(21)$ & $-119.3(5)$ \\
\hline $\mathrm{C}(19)$ & $\mathrm{Fe}(1)$ & $\mathrm{C}(21)$ & $C(20)$ & $38.0(4)$ & $\mathrm{C}(19)$ & $\mathrm{Fe}(1)$ & $\mathrm{C}(21)$ & $\mathrm{C}(22)$ & $-81.9(4)$ \\
\hline $\mathrm{C}(19)$ & $\mathrm{Fe}(1)$ & $\mathrm{C}(22)$ & $\mathrm{C}(21)$ & $81.6(4)$ & $\mathrm{C}(19)$ & $\mathrm{Fe}(1)$ & $C(22)$ & $C(23)$ & $-37.4(3)$ \\
\hline
\end{tabular}


Table S12. Torsion Angles( $\left(^{\circ}\right)$ for 5 (continued)

\begin{tabular}{|c|c|c|c|c|c|c|c|c|c|}
\hline $\mathrm{m}$ & 1 & atom & n & angle & $n$ & $n$ & $\mathrm{~m}$ & $\mathrm{~m}$ & e \\
\hline$C(19)$ & $\mathrm{Fe}(1)$ & $\mathrm{C}(23)$ & $\mathrm{C}(22)$ & $120.2(5)$ & $\mathrm{C}(19)$ & $\mathrm{Fe}(1)$ & $\mathrm{C}(23)$ & $C(29)$ & $-116.8(7)$ \\
\hline$C(19)$ & $\mathrm{Fe}(1)$ & $\mathrm{C}(24)$ & $\mathrm{C}(25)$ & $-83.0(5)$ & $\mathrm{C}(19)$ & $\mathrm{Fe}(1)$ & $\mathrm{C}(24)$ & $C(28)$ & $158.3(4)$ \\
\hline $\mathrm{C}(19)$ & $\mathrm{Fe}(1)$ & $\mathrm{C}(25)$ & $\mathrm{C}(24)$ & 119.1(4) & $\mathrm{C}(19)$ & $\mathrm{Fe}(1)$ & $\mathrm{C}(25)$ & $C(26)$ & $-121.9(4)$ \\
\hline$C(19)$ & $\mathrm{Fe}(1)$ & $\mathrm{C}(26)$ & $\mathrm{C}(25)$ & $80.4(5)$ & $\mathrm{C}(19)$ & $\mathrm{Fe}(1)$ & $C(26)$ & $C(27)$ & $-159.5(4)$ \\
\hline$C(19)$ & $\mathrm{Fe}(1)$ & $\mathrm{C}(27)$ & $C(26)$ & $58(1)$ & $\mathrm{C}(19)$ & $\mathrm{Fe}(1)$ & $\mathrm{C}(27)$ & $\mathrm{C}(28)$ & $175.9(8)$ \\
\hline$C(19)$ & $\mathrm{Fe}(1)$ & $\mathrm{C}(28)$ & $\mathrm{C}(24)$ & $-55.8(10)$ & $\mathrm{C}(19)$ & $\mathrm{Fe}(1)$ & $\mathrm{C}(28)$ & $C(27)$ & $-176.3(7)$ \\
\hline$C(19)$ & $C(20)$ & $\mathrm{Fe}(1)$ & $\mathrm{C}(21)$ & $119.3(5)$ & $\mathrm{C}(19)$ & $C(20)$ & $\mathrm{Fe}(1)$ & $C(22)$ & $82.1(4)$ \\
\hline$C(19)$ & $C(20)$ & $\mathrm{Fe}(1)$ & $\mathrm{C}(23)$ & 38. & $\mathrm{C}(19)$ & $\mathrm{C}(20)$ & $\mathrm{Fe}(1)$ & $C(24)$ & $-58.2(8)$ \\
\hline$C(19)$ & $\mathrm{C}(20)$ & $\mathrm{Fe}(1)$ & $\mathrm{C}(25)$ & $-87.6(4)$ & $\mathrm{C}(19)$ & $\mathrm{C}(20)$ & $\mathrm{Fe}(1)$ & $C(26)$ & $-128.6(4)$ \\
\hline$C(19)$ & $\mathrm{C}(20)$ & $\mathrm{Fe}(1)$ & $\mathrm{C}(27)$ & $-170.3(4)$ & $\mathrm{C}(19)$ & $\mathrm{C}(20)$ & $\mathrm{Fe}(1)$ & $C(28)$ & $161.5(7)$ \\
\hline$C(19)$ & $\mathrm{C}(20)$ & $\mathrm{C}(21)$ & $\mathrm{C}(22)$ & $-0.6(7)$ & $\mathrm{C}(19)$ & $\mathrm{C}(23)$ & $\mathrm{Fe}(1)$ & $C(20)$ & $-38.7(4)$ \\
\hline$C(19)$ & $\mathrm{C}(23)$ & $\mathrm{Fe}(1)$ & $\mathrm{C}(21)$ & $-82.4(4)$ & $\mathrm{C}(19)$ & $\mathrm{C}(23)$ & $\mathrm{Fe}(1)$ & $C(22)$ & $-120.2(5)$ \\
\hline$C(19)$ & $\mathrm{C}(23)$ & $\mathrm{Fe}(1)$ & $\mathrm{C}(24)$ & $118.3(4)$ & $\mathrm{C}(19)$ & $\mathrm{C}(23)$ & $\mathrm{Fe}(1)$ & $C(25)$ & $75.0(5)$ \\
\hline$C(19)$ & $\mathrm{C}(23)$ & $\mathrm{Fe}(1)$ & $C(26)$ & $28(1)$ & $\mathrm{C}(19)$ & $\mathrm{C}(23)$ & $\mathrm{Fe}(1)$ & $C(27)$ & $-155.0(6)$ \\
\hline$C(19)$ & $\mathrm{C}(23)$ & $\mathrm{Fe}(1)$ & $\mathrm{C}(28)$ & $162.8(4)$ & $\mathrm{C}(19)$ & $\mathrm{C}(23)$ & $\mathrm{C}(22)$ & $\mathrm{C}(21)$ & $-2.6(7)$ \\
\hline$C(19)$ & $\mathrm{C}(23)$ & $\mathrm{C}(29)$ & $\mathrm{C}(30)$ & $-155.1(5)$ & $\mathrm{C}(20)$ & $\mathrm{Fe}(1)$ & $\mathrm{C}(19)$ & $\mathrm{C}(23)$ & $117.9(5)$ \\
\hline $\mathrm{C}(20)$ & $\mathrm{Fe}(1)$ & $\mathrm{C}(21)$ & $\mathrm{C}(22)$ & $-119.9(5)$ & $\mathrm{C}(20)$ & $\mathrm{Fe}(1)$ & $\mathrm{C}(22)$ & $\mathrm{C}(21)$ & $37.3(4)$ \\
\hline $\mathrm{C}(20)$ & $\mathrm{Fe}(1)$ & $\mathrm{C}(22)$ & $\mathrm{C}(23)$ & $-81.7(4)$ & $\mathrm{C}(20)$ & $\mathrm{Fe}(1)$ & $\mathrm{C}(23)$ & $C(22)$ & $81.5(4)$ \\
\hline $\mathrm{C}(20)$ & $\mathrm{Fe}(1)$ & $\mathrm{C}(23)$ & C(29) & $-155.5(7)$ & $\mathrm{C}(20)$ & $\mathrm{Fe}(1)$ & $\mathrm{C}(24)$ & $C(25)$ & $9.8(9)$ \\
\hline$C(20)$ & $\mathrm{Fe}(1)$ & $\mathrm{C}(24)$ & $\mathrm{C}(28)$ & $-158.6(6)$ & $\mathrm{C}(20)$ & $\mathrm{Fe}(1)$ & $\mathrm{C}(25)$ & $C(24)$ & 1(4) \\
\hline$C(20)$ & $\mathrm{Fe}(1)$ & $\mathrm{C}(25)$ & $C(26)$ & $-76.9(5)$ & $\mathrm{C}(20)$ & $\mathrm{Fe}(1)$ & $C(26)$ & $C(25)$ & $120.4(5)$ \\
\hline $\mathrm{C}(20)$ & $\mathrm{Fe}(1)$ & $C(26)$ & $\mathrm{C}(27)$ & $-119.6(5)$ & $C(20)$ & $\mathrm{Fe}(1)$ & $\mathrm{C}(27)$ & $C(26)$ & $77.8(5)$ \\
\hline $\mathrm{C}(20)$ & $\mathrm{Fe}(1)$ & $\mathrm{C}(27)$ & $\mathrm{C}(28)$ & $-164.4(5)$ & $\mathrm{C}(20)$ & $\mathrm{Fe}(1)$ & $\mathrm{C}(28)$ & $C(24)$ & $159.0(7)$ \\
\hline $\mathrm{C}(20)$ & $\mathrm{Fe}(1)$ & $C(28)$ & $\mathrm{C}(27)$ & $38(1)$ & $\mathrm{C}(20)$ & $\mathrm{C}(19)$ & $\mathrm{Fe}(1)$ & $\mathrm{C}(21)$ & $-37.4(4)$ \\
\hline $\mathrm{C}(20)$ & $\mathrm{C}(19)$ & $\mathrm{Fe}(1)$ & $\mathrm{C}(22)$ & $-81.0(4)$ & $\mathrm{C}(20)$ & $\mathrm{C}(19)$ & $\mathrm{Fe}(1)$ & $\mathrm{C}(23)$ & $-117.9(5)$ \\
\hline $\mathrm{C}(20)$ & $\mathrm{C}(19)$ & $\mathrm{Fe}(1)$ & $\mathrm{C}(24)$ & $157.8(4)$ & $\mathrm{C}(20)$ & $\mathrm{C}(19)$ & $\mathrm{Fe}(1)$ & $C(25)$ & $113.5(4)$ \\
\hline $\mathrm{C}(20)$ & $\mathrm{C}(19)$ & $\mathrm{Fe}(1)$ & $C(26)$ & $70.0(5)$ & $\mathrm{C}(20)$ & $\mathrm{C}(19)$ & $\mathrm{Fe}(1)$ & $C(27)$ & $26(1)$ \\
\hline$C(20)$ & $\mathrm{C}(19)$ & $\mathrm{Fe}(1)$ & $\mathrm{C}(28)$ & $-161.2(8)$ & $\mathrm{C}(20)$ & $\mathrm{C}(19)$ & $\mathrm{C}(23)$ & $C(22)$ & $2.2(6)$ \\
\hline $\mathrm{C}(20)$ & $\mathrm{C}(19)$ & $\mathrm{C}(23)$ & $\mathrm{C}(29)$ & $-174.5(5)$ & $\mathrm{C}(20)$ & $\mathrm{C}(21)$ & $\mathrm{Fe}(1)$ & $\mathrm{C}(22)$ & $119.9(5)$ \\
\hline $\mathrm{C}(20)$ & $\mathrm{C}(21)$ & $\mathrm{Fe}(1)$ & $\mathrm{C}(23)$ & $82.2(4)$ & $\mathrm{C}(20)$ & $\mathrm{C}(21)$ & $\mathrm{Fe}(1)$ & $C(24)$ & $177.3(8)$ \\
\hline $\mathrm{C}(20)$ & $\mathrm{C}(21)$ & $\mathrm{Fe}(1)$ & $C(25)$ & $-58.3(7)$ & $\mathrm{C}(20)$ & $\mathrm{C}(21)$ & $\mathrm{Fe}(1)$ & $C(26)$ & $-83.8(4)$ \\
\hline
\end{tabular}


Table S12. Torsion Angles( $\left(^{\circ}\right)$ for 5 (continued)

\begin{tabular}{|c|c|c|c|c|c|c|c|c|c|}
\hline $\mathrm{m}$ & m & tom & atom & angle & $n$ & $\mathrm{~m}$ & $\mathrm{~m}$ & $\mathrm{n}$ & e \\
\hline$C(20)$ & $\mathrm{C}(21)$ & $\mathrm{Fe}(1)$ & $\mathrm{C}(27)$ & $-124.5(4)$ & $\mathrm{C}(20)$ & $\mathrm{C}(21)$ & $\mathrm{Fe}(1)$ & $\mathrm{C}(28)$ & $163.0(4)$ \\
\hline $\mathrm{C}(20)$ & $\mathrm{C}(21)$ & $\mathrm{C}(22)$ & $\mathrm{C}(23)$ & $2.0(7)$ & $\mathrm{C}(21)$ & $\mathrm{Fe}(1)$ & $\mathrm{C}(19)$ & $\mathrm{C}(23)$ & $80.5(4)$ \\
\hline $\mathrm{C}(21)$ & $\mathrm{Fe}(1)$ & $\mathrm{C}(22)$ & $\mathrm{C}(23)$ & $-119.0(5)$ & $\mathrm{C}(21)$ & $\mathrm{Fe}(1)$ & $\mathrm{C}(23)$ & $\mathrm{C}(22)$ & $37.8(4)$ \\
\hline $\mathrm{C}(21)$ & $\mathrm{Fe}(1)$ & $\mathrm{C}(23)$ & C(29) & $160.8(7)$ & $\mathrm{C}(21)$ & $\mathrm{Fe}(1)$ & $\mathrm{C}(24)$ & $\mathrm{C}(25)$ & $145.0(8)$ \\
\hline $\mathrm{C}(21)$ & $\mathrm{Fe}(1)$ & $\mathrm{C}(24)$ & $\mathrm{C}(28)$ & $26(1)$ & $\mathrm{C}(21)$ & $\mathrm{Fe}(1)$ & $C(25)$ & $\mathrm{C}(24)$ & $-155.4(6)$ \\
\hline $\mathrm{C}(21)$ & $\mathrm{Fe}(1)$ & $\mathrm{C}(25)$ & $C(26)$ & $-36.5(8)$ & $\mathrm{C}(21)$ & $\mathrm{Fe}(1)$ & $C(26)$ & $\mathrm{C}(25)$ & $162.5(4)$ \\
\hline$C(21)$ & $\mathrm{Fe}(1)$ & $C(26)$ & $\mathrm{C}(27)$ & $-77.4(5)$ & $\mathrm{C}(21)$ & $\mathrm{Fe}(1)$ & $\mathrm{C}(27)$ & $C(26)$ & $116.5(5)$ \\
\hline $\mathrm{C}(21)$ & $\mathrm{Fe}(1)$ & $\mathrm{C}(27)$ & $\mathrm{C}(28)$ & $-125.7(5)$ & $\mathrm{C}(21)$ & $\mathrm{Fe}(1)$ & $\mathrm{C}(28)$ & $\mathrm{C}(24)$ & $-170.1(4)$ \\
\hline $\mathrm{C}(21)$ & $\mathrm{Fe}(1)$ & $\mathrm{C}(28)$ & $\mathrm{C}(27)$ & $69.4(5)$ & $\mathrm{C}(21)$ & $C(20)$ & $\mathrm{Fe}(1)$ & $\mathrm{C}(22)$ & $-37.2(4)$ \\
\hline$C(21)$ & $\mathrm{C}(20)$ & $\mathrm{Fe}(1)$ & $\mathrm{C}(23)$ & $-80.8(4)$ & $\mathrm{C}(21)$ & $\mathrm{C}(20)$ & $\mathrm{Fe}(1)$ & $\mathrm{C}(24)$ & $-177.5(7)$ \\
\hline $\mathrm{C}(21)$ & $\mathrm{C}(20)$ & $\mathrm{Fe}(1)$ & $C(25)$ & $153.1(4)$ & $\mathrm{C}(21)$ & $\mathrm{C}(20)$ & $\mathrm{Fe}(1)$ & $C(26)$ & $112.1(4)$ \\
\hline $\mathrm{C}(21)$ & $\mathrm{C}(20)$ & $\mathrm{Fe}(1)$ & $\mathrm{C}(27)$ & $70.4(5)$ & $\mathrm{C}(21)$ & $\mathrm{C}(20)$ & $\mathrm{Fe}(1)$ & $\mathrm{C}(28)$ & $42.2(9)$ \\
\hline$C(21)$ & $\mathrm{C}(20)$ & $\mathrm{C}(19)$ & $\mathrm{C}(23)$ & $-1.0(6)$ & $\mathrm{C}(21)$ & $\mathrm{C}(22)$ & $\mathrm{Fe}(1)$ & $\mathrm{C}(23)$ & $119.0(5)$ \\
\hline $\mathrm{C}(21)$ & $\mathrm{C}(22)$ & $\mathrm{Fe}(1)$ & $\mathrm{C}(24)$ & $-159.6(4)$ & $\mathrm{C}(21)$ & $C(22)$ & $\mathrm{Fe}(1)$ & $C(25)$ & $176(1)$ \\
\hline $\mathrm{C}(21)$ & $\mathrm{C}(22)$ & $\mathrm{Fe}(1)$ & $C(26)$ & $-46.7(7)$ & $\mathrm{C}(21)$ & $C(22)$ & $\mathrm{Fe}(1)$ & $\mathrm{C}(27)$ & $-78.0(4)$ \\
\hline $\mathrm{C}(21)$ & $\mathrm{C}(22)$ & $\mathrm{Fe}(1)$ & $\mathrm{C}(28)$ & $-120.5(4)$ & $\mathrm{C}(21)$ & $C(22)$ & $\mathrm{C}(23)$ & C(29) & $173.9(5)$ \\
\hline$C(22)$ & $\mathrm{Fe}(1)$ & C(19) & $\mathrm{C}(23)$ & $36.9(4)$ & $\mathrm{C}(22)$ & $\mathrm{Fe}(1)$ & $\mathrm{C}(23)$ & C(29) & $123.0(7)$ \\
\hline $\mathrm{C}(22)$ & $\mathrm{Fe}(1)$ & $\mathrm{C}(24)$ & $\mathrm{C}(25)$ & $-171.7(4)$ & $\mathrm{C}(22)$ & $\mathrm{Fe}(1)$ & $\mathrm{C}(24)$ & $\mathrm{C}(28)$ & $69.5(5)$ \\
\hline$C(22)$ & $\mathrm{Fe}(1)$ & $\mathrm{C}(25)$ & $\mathrm{C}(24)$ & $29(1)$ & $\mathrm{C}(22)$ & $\mathrm{Fe}(1)$ & $\mathrm{C}(25)$ & $C(26)$ & $148(1)$ \\
\hline$C(22)$ & $\mathrm{Fe}(1)$ & $C(26)$ & $\mathrm{C}(25)$ & $-165.7(5)$ & $\mathrm{C}(22)$ & $\mathrm{Fe}(1)$ & $C(26)$ & $\mathrm{C}(27)$ & $-45.7(7)$ \\
\hline$C(22)$ & $\mathrm{Fe}(1)$ & $\mathrm{C}(27)$ & $C(26)$ & $156.9(4)$ & $\mathrm{C}(22)$ & $\mathrm{Fe}(1)$ & $\mathrm{C}(27)$ & $\mathrm{C}(28)$ & $-85.3(5)$ \\
\hline $\mathrm{C}(22)$ & $\mathrm{Fe}(1)$ & $\mathrm{C}(28)$ & $\mathrm{C}(24)$ & $-128.9(5)$ & $\mathrm{C}(22)$ & $\mathrm{Fe}(1)$ & $\mathrm{C}(28)$ & $\mathrm{C}(27)$ & $110.6(5)$ \\
\hline$C(22)$ & $\mathrm{C}(21)$ & $\mathrm{Fe}(1)$ & $\mathrm{C}(23)$ & $-37.7(4)$ & $\mathrm{C}(22)$ & $\mathrm{C}(21)$ & $\mathrm{Fe}(1)$ & $\mathrm{C}(24)$ & $57.4(10)$ \\
\hline$C(22)$ & $\mathrm{C}(21)$ & $\mathrm{Fe}(1)$ & $\mathrm{C}(25)$ & $-178.2(6)$ & $\mathrm{C}(22)$ & $\mathrm{C}(21)$ & $\mathrm{Fe}(1)$ & $C(26)$ & $156.4(4)$ \\
\hline$C(22)$ & $\mathrm{C}(21)$ & $\mathrm{Fe}(1)$ & $\mathrm{C}(27)$ & 115.7(4) & $\mathrm{C}(22)$ & $\mathrm{C}(21)$ & $\mathrm{Fe}(1)$ & $\mathrm{C}(28)$ & $77.1(5)$ \\
\hline$C(22)$ & $\mathrm{C}(23)$ & $\mathrm{Fe}(1)$ & $\mathrm{C}(24)$ & $-121.5(4)$ & $\mathrm{C}(22)$ & $\mathrm{C}(23)$ & $\mathrm{Fe}(1)$ & $\mathrm{C}(25)$ & $-164.8(4)$ \\
\hline$C(22)$ & $\mathrm{C}(23)$ & $\mathrm{Fe}(1)$ & $C(26)$ & $148(1)$ & $\mathrm{C}(22)$ & $\mathrm{C}(23)$ & $\mathrm{Fe}(1)$ & $\mathrm{C}(27)$ & $-34.9(7)$ \\
\hline$C(22)$ & $\mathrm{C}(23)$ & $\mathrm{Fe}(1)$ & $\mathrm{C}(28)$ & $-77.0(5)$ & $\mathrm{C}(22)$ & $\mathrm{C}(23)$ & $\mathrm{C}(29)$ & $C(30)$ & $29.0(8)$ \\
\hline $\mathrm{C}(23)$ & $\mathrm{Fe}(1)$ & $\mathrm{C}(24)$ & $\mathrm{C}(25)$ & $-127.8(4)$ & $\mathrm{C}(23)$ & $\mathrm{Fe}(1)$ & $\mathrm{C}(24)$ & $\mathrm{C}(28)$ & $113.4(5)$ \\
\hline$C(23)$ & $\mathrm{Fe}(1)$ & $C(25)$ & $\mathrm{C}(24)$ & $76.2(5)$ & $\mathrm{C}(23)$ & $\mathrm{Fe}(1)$ & $\mathrm{C}(25)$ & $C(26)$ & $-164.8(4)$ \\
\hline
\end{tabular}


Table S12. Torsion Angles( $\left(^{\circ}\right)$ for 5 (continued)

\begin{tabular}{|c|c|c|c|c|c|c|c|c|c|}
\hline $\mathrm{m}$ & $\mathrm{m}$ & tom & atom & angle & $\mathrm{n}$ & $n$ & $\mathrm{~m}$ & $\mathrm{~m}$ & e \\
\hline (23) & $\mathrm{Fe}(1)$ & $C(26)$ & $C(25)$ & $57(1)$ & $\mathrm{C}(23)$ & $\mathrm{Fe}(1)$ & $C(26)$ & $\mathrm{C}(27)$ & $177.7(10)$ \\
\hline$C(23)$ & $\mathrm{Fe}(1)$ & $\mathrm{C}(27)$ & $C(26)$ & $-178.8(5)$ & $\mathrm{C}(23)$ & $\mathrm{Fe}(1)$ & $\mathrm{C}(27)$ & $\mathrm{C}(28)$ & $-61.0(8)$ \\
\hline $\mathrm{C}(23)$ & $\mathrm{Fe}(1)$ & $\mathrm{C}(28)$ & $\mathrm{C}(24)$ & $-87.8(5)$ & $\mathrm{C}(23)$ & $\mathrm{Fe}(1)$ & $\mathrm{C}(28)$ & $\mathrm{C}(27)$ & $151.7(5)$ \\
\hline $\mathrm{C}(23)$ & $\mathrm{C}(19)$ & $\mathrm{Fe}(1)$ & $\mathrm{C}(24)$ & $-84.3(5)$ & $\mathrm{C}(23)$ & $C(19)$ & $\mathrm{Fe}(1)$ & $C(25)$ & $-128.6(4)$ \\
\hline $\mathrm{C}(23)$ & $\mathrm{C}(19)$ & $\mathrm{Fe}(1)$ & $C(26)$ & $-172.1(4)$ & $\mathrm{C}(23)$ & $C(19)$ & $\mathrm{Fe}(1)$ & $\mathrm{C}(27)$ & $144.0(9)$ \\
\hline $\mathrm{C}(23)$ & $\mathrm{C}(19)$ & $\mathrm{Fe}(1)$ & $\mathrm{C}(28)$ & $-43.3(9)$ & $\mathrm{C}(23)$ & $C(22)$ & $\mathrm{Fe}(1)$ & $\mathrm{C}(24)$ & $81.4(5)$ \\
\hline$(23)$ & $\mathrm{C}(22)$ & $\mathrm{Fe}(1)$ & $C(25)$ & $57(1)$ & $\mathrm{C}(23)$ & $C(22)$ & $\mathrm{Fe}(1)$ & $C(26)$ & $165.7(5)$ \\
\hline (23) & $\mathrm{C}(22)$ & $\mathrm{Fe}(1)$ & $\mathrm{C}(27)$ & $163.1(4)$ & $\mathrm{C}(23)$ & $C(22)$ & $\mathrm{Fe}(1)$ & $\mathrm{C}(28)$ & $120.6(4)$ \\
\hline (23) & C(29) & $\mathrm{P}(2)$ & $\mathrm{C}(31)$ & $-176.8(4)$ & $\mathrm{C}(23)$ & $C(29)$ & $\mathrm{P}(2)$ & $C(35)$ & $64.9(5)$ \\
\hline$C(24)$ & $\mathrm{Fe}(1)$ & $\mathrm{C}(23)$ & $\mathrm{C}(29)$ & $1.5(7)$ & $\mathrm{C}(24)$ & $\mathrm{Fe}(1)$ & $C(25)$ & $C(26)$ & $119.0(6)$ \\
\hline $\mathrm{C}(24)$ & $\mathrm{Fe}(1)$ & $C(26)$ & $\mathrm{C}(25)$ & $-38.0(4)$ & $\mathrm{C}(24)$ & $\mathrm{Fe}(1)$ & $C(26)$ & $\mathrm{C}(27)$ & $82.1(5)$ \\
\hline $\mathrm{C}(24)$ & $\mathrm{Fe}(1)$ & $\mathrm{C}(27)$ & $C(26)$ & $-81.0(5)$ & $\mathrm{C}(24)$ & $\mathrm{Fe}(1)$ & $\mathrm{C}(27)$ & $\mathrm{C}(28)$ & $36.8(5)$ \\
\hline$C(24)$ & $\mathrm{Fe}(1)$ & $\mathrm{C}(28)$ & $\mathrm{C}(27)$ & $-120.5(7)$ & $C(24)$ & $C(25)$ & $\mathrm{Fe}(1)$ & $C(26)$ & $-119.0(6)$ \\
\hline$C(24)$ & $\mathrm{C}(25)$ & $\mathrm{Fe}(1)$ & $\mathrm{C}(27)$ & $-81.6(5)$ & $\mathrm{C}(24)$ & $C(25)$ & $\mathrm{Fe}(1)$ & $\mathrm{C}(28)$ & $-37.7(4)$ \\
\hline $\mathrm{C}(24)$ & $\mathrm{C}(25)$ & $C(26)$ & $\mathrm{C}(27)$ & $1.1(7)$ & $\mathrm{C}(24)$ & $\mathrm{C}(28)$ & $\mathrm{Fe}(1)$ & $C(25)$ & $38.1(5)$ \\
\hline$C(24)$ & $\mathrm{C}(28)$ & $\mathrm{Fe}(1)$ & $C(26)$ & $81.9(5)$ & $\mathrm{C}(24)$ & $\mathrm{C}(28)$ & $\mathrm{Fe}(1)$ & $\mathrm{C}(27)$ & $120.5(7)$ \\
\hline $\mathrm{C}(24)$ & $\mathrm{C}(28)$ & $\mathrm{C}(27)$ & $C(26)$ & $1.6(8)$ & $\mathrm{C}(25)$ & $\mathrm{Fe}(1)$ & $\mathrm{C}(23)$ & $\mathrm{C}(29)$ & $-41.8(7)$ \\
\hline$C(25)$ & $\mathrm{Fe}(1)$ & $\mathrm{C}(24)$ & $\mathrm{C}(28)$ & $-118.7(6)$ & $\mathrm{C}(25)$ & $\mathrm{Fe}(1)$ & $C(26)$ & $\mathrm{C}(27)$ & $120.0(6)$ \\
\hline$C(25)$ & $\mathrm{Fe}(1)$ & $\mathrm{C}(27)$ & $C(26)$ & $-37.0(4)$ & $\mathrm{C}(25)$ & $\mathrm{Fe}(1)$ & $\mathrm{C}(27)$ & $\mathrm{C}(28)$ & $80.8(5)$ \\
\hline$C(25)$ & $\mathrm{Fe}(1)$ & $\mathrm{C}(28)$ & $\mathrm{C}(27)$ & $-82.3(5)$ & $C(25)$ & $C(24)$ & $\mathrm{Fe}(1)$ & $C(26)$ & $37.6(4)$ \\
\hline$C(25)$ & $\mathrm{C}(24)$ & $\mathrm{Fe}(1)$ & $\mathrm{C}(27)$ & $81.7(5)$ & $C(25)$ & $C(24)$ & $\mathrm{Fe}(1)$ & $\mathrm{C}(28)$ & $118.7(6)$ \\
\hline$C(25)$ & $\mathrm{C}(24)$ & $\mathrm{C}(28)$ & $\mathrm{C}(27)$ & $-1.0(8)$ & $\mathrm{C}(25)$ & $C(26)$ & $\mathrm{Fe}(1)$ & $\mathrm{C}(27)$ & $-120.0(6)$ \\
\hline$C(25)$ & $C(26)$ & $\mathrm{Fe}(1)$ & $\mathrm{C}(28)$ & $-81.6(5)$ & $\mathrm{C}(25)$ & $C(26)$ & $\mathrm{C}(27)$ & $\mathrm{C}(28)$ & $-1.7(7)$ \\
\hline$C(26)$ & $\mathrm{Fe}(1)$ & $\mathrm{C}(23)$ & $\mathrm{C}(29)$ & $-88(1)$ & $C(26)$ & $\mathrm{Fe}(1)$ & $\mathrm{C}(24)$ & $\mathrm{C}(28)$ & $-81.1(5)$ \\
\hline$C(26)$ & $\mathrm{Fe}(1)$ & $\mathrm{C}(27)$ & $\mathrm{C}(28)$ & 117.8(7) & $C(26)$ & $\mathrm{Fe}(1)$ & $\mathrm{C}(28)$ & $\mathrm{C}(27)$ & $-38.6(5)$ \\
\hline$C(26)$ & $\mathrm{C}(25)$ & $\mathrm{Fe}(1)$ & $\mathrm{C}(27)$ & $37.4(4)$ & $C(26)$ & $C(25)$ & $\mathrm{Fe}(1)$ & $\mathrm{C}(28)$ & $81.2(5)$ \\
\hline$C(26)$ & $\mathrm{C}(25)$ & $\mathrm{C}(24)$ & $\mathrm{C}(28)$ & $-0.1(7)$ & $C(26)$ & $C(27)$ & $\mathrm{Fe}(1)$ & $\mathrm{C}(28)$ & $-117.8(7)$ \\
\hline$C(27)$ & $\mathrm{Fe}(1)$ & $\mathrm{C}(23)$ & $C(29)$ & $88.1(8)$ & $\mathrm{C}(27)$ & $\mathrm{Fe}(1)$ & $\mathrm{C}(24)$ & $\mathrm{C}(28)$ & $-37.0(5)$ \\
\hline $\mathrm{C}(27)$ & $C(26)$ & $\mathrm{Fe}(1)$ & $\mathrm{C}(28)$ & $38.4(5)$ & $\mathrm{C}(28)$ & $\mathrm{Fe}(1)$ & $\mathrm{C}(23)$ & $\mathrm{C}(29)$ & $46.0(7)$ \\
\hline$C(29)$ & $\mathrm{P}(2)$ & $\mathrm{C}(31)$ & $C(32)$ & $162.1(5)$ & $\mathrm{C}(29)$ & $\mathrm{P}(2)$ & $\mathrm{C}(31)$ & $\mathrm{C}(33)$ & $47.4(5)$ \\
\hline C(29) & $\mathrm{P}(2)$ & $\mathrm{C}(31)$ & $\mathrm{C}(34)$ & $-74.9(5)$ & $\mathrm{C}(29)$ & $\mathrm{P}(2)$ & $\mathrm{C}(35)$ & $C(36)$ & $-86.4(5)$ \\
\hline$C(29)$ & $\mathrm{P}(2)$ & $C(35)$ & $\mathrm{C}(37)$ & $32.8(5)$ & $\mathrm{C}(29)$ & $\mathrm{P}(2)$ & $C(35)$ & $\mathrm{C}(38)$ & $157.5(4)$ \\
\hline
\end{tabular}


Table S12. Torsion Angles $\left({ }^{\circ}\right)$ for 5 (continued)

$\begin{array}{llllllllll}\text { atom } & \text { atom } & \text { atom } & \text { atom } & \text { angle } & \text { atom } & \text { atom } & \text { atom } & \text { atom } & \text { angle } \\ \mathrm{C}(30) & \mathrm{C}(29) & \mathrm{P}(2) & \mathrm{C}(31) & 51.9(6) & \mathrm{C}(30) & \mathrm{C}(29) & \mathrm{P}(2) & \mathrm{C}(35) & -66.5(6) \\ \mathrm{C}(31) & \mathrm{P}(2) & \mathrm{C}(35) & \mathrm{C}(36) & 161.4(4) & \mathrm{C}(31) & \mathrm{P}(2) & \mathrm{C}(35) & \mathrm{C}(37) & -79.4(5) \\ \mathrm{C}(31) & \mathrm{P}(2) & \mathrm{C}(35) & \mathrm{C}(38) & 45.3(5) & \mathrm{C}(32) & \mathrm{C}(31) & \mathrm{P}(2) & \mathrm{C}(35) & -81.0(6) \\ \mathrm{C}(33) & \mathrm{C}(31) & \mathrm{P}(2) & \mathrm{C}(35) & 164.3(4) & \mathrm{C}(34) & \mathrm{C}(31) & \mathrm{P}(2) & \mathrm{C}(35) & 42.0(6) \\ \mathrm{C}(40) & \mathrm{O}(1) & \mathrm{C}(43) & \mathrm{C}(42) & 7(1) & \mathrm{C}(40) & \mathrm{C}(41) & \mathrm{C}(42) & \mathrm{C}(43) & 27(1) \\ \mathrm{C}(41) & \mathrm{C}(40) & \mathrm{O}(1) & \mathrm{C}(43) & 11(1) & & & & & \end{array}$


Table S13. Non-bonded Contacts out to $3.60 \AA$ for 5

$\begin{array}{llllllll}\text { atom } & \text { atom } & \text { distance } & \text { ADC } & \text { atom } & \text { atom } & \text { distance } & \text { ADC } \\ \mathrm{O}(1) & \mathrm{C}(21) & 3.45(1) & 1 & \mathrm{O}(1) & \mathrm{C}(12) & 3.454(9) & 35302 \\ \mathrm{O}(1) & \mathrm{C}(25) & 3.59(1) & 35302 & \mathrm{C}(2) & \mathrm{C}(17) & 3.469(8) & 65501 \\ \mathrm{C}(3) & \mathrm{C}(16) & 3.508(8) & 65501 & \mathrm{C}(3) & \mathrm{C}(17) & 3.590(9) & 65501 \\ \mathrm{C}(5) & \mathrm{C}(38) & 3.561(9) & 44402 & \mathrm{C}(33) & \mathrm{C}(37) & 3.58(1) & 34402\end{array}$

The ADC (atom designator code) specifies the position of an atom in a crystal. The 5-digit number shown in the table is a composite of three one-digit numbers and one two-digit number: TA (first digit) + TB (second digit) + TC (third digit) + SN (last two digits). TA, TB and TC are the crystal lattice translation digits along cell edges $\mathrm{a}, \mathrm{b}$ and $\mathrm{c}$. A translation digit of 5 indicates the origin unit cell. If $\mathrm{TA}=4$, this indicates a translation of one unit cell length along the a-axis in the negative direction. Each translation digit can range in value from 1 to 9 and thus \pm 4 lattice translations from the origin $(\mathrm{TA}=5, \mathrm{~TB}=5, \mathrm{TC}=5)$ can be represented.

The SN, or symmetry operator number, refers to the number of the symmetry operator used to generate the coordinates of the target atom. A list of symmetry operators relevant to this structure are given below.

For a given intermolecular contact, the first atom (origin atom) is located in the origin unit cell and its position can be generated using the identity operator $(\mathrm{SN}=1)$. Thus, the ADC for an origin atom is always 55501. The position of the second atom (target atom) can be generated using the $\mathrm{ADC}$ and the coordinates of the atom in the parameter table. For example, an ADC of 47502 refers to the target atom moved through symmetry operator two, then translated -1 cell translations along the a axis, +2 cell translations along the $b$ axis, and 0 cell translations along the c axis.

An ADC of 1 indicates an intermolecular contact between two fragments (eg. cation and anion) that reside in the same asymmetric unit.

Symmetry Operators:

(1) $\mathrm{X}, \mathrm{Y}, \mathrm{Z} \quad$ (2) $-\mathrm{X}, \quad 1 / 2+\mathrm{Y},-\mathrm{Z}$ 


\section{Crystallographic data for 6.}

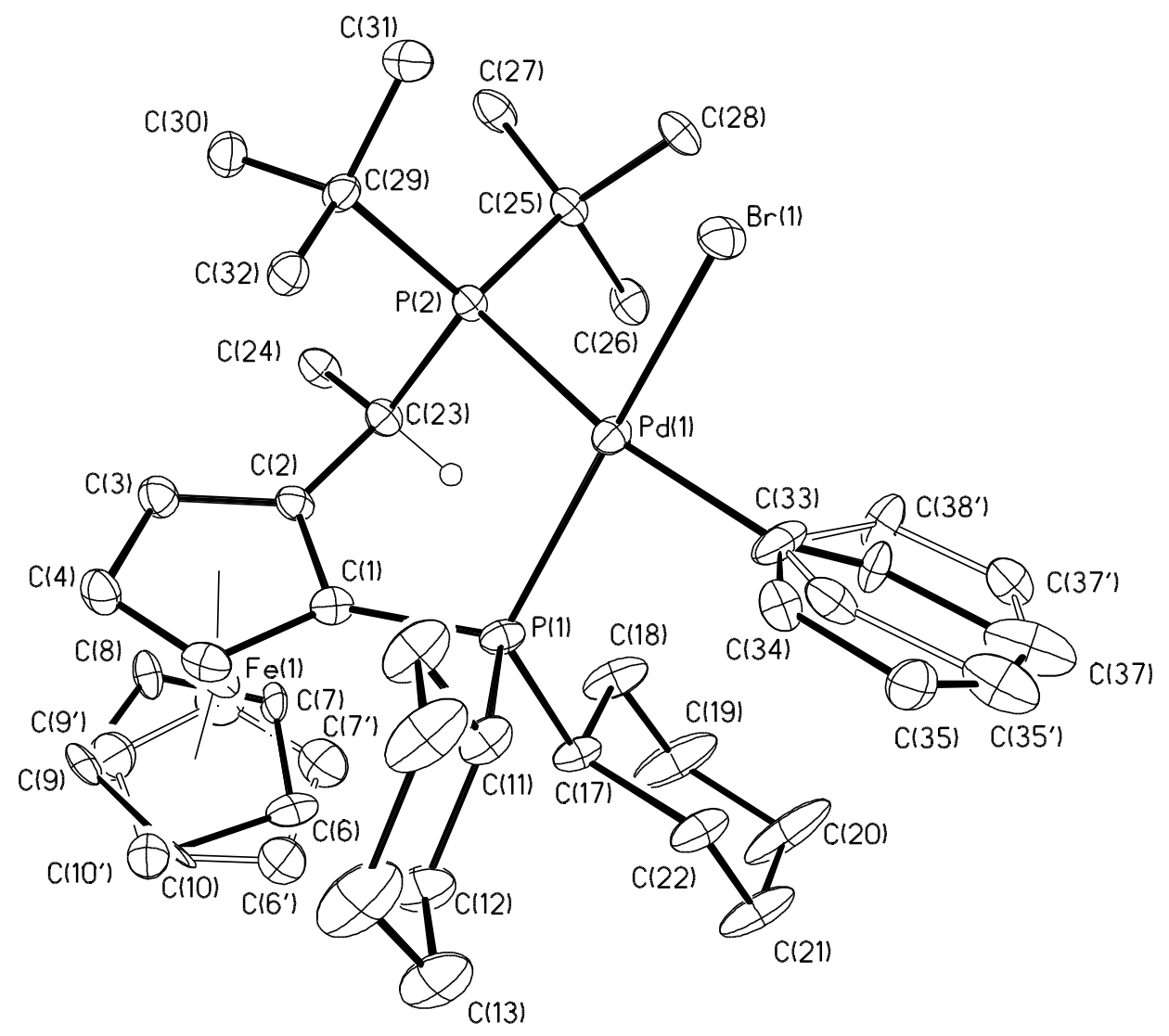

Figure S7. X-ray crystal structure of 6

\section{Data Collection}

The data were collected on a Nonius KappaCCD diffractometer with graphite monochromated Mo-K $\alpha$ radiation at a temperature of 183(2) $\mathrm{K}$ to a maximum $2 \theta$ value of 56.56

o. Five omega scans consisting of 37, 37, 19, 19, and 20 data frames, respectively, were collected with a frame width of $2.0^{\circ}$ and a detector-to-crystal distance, Dx, of $35 \mathrm{~mm}$. Each frame was exposed twice (for the purpose of de-zingering) for a total of 60 seconds. The data frames were processed and scaled using the DENZO software package. ${ }^{1}$ 


\section{$\underline{\text { Structure Solution and Refinement }}$}

The structure was solved by direct methods and expanded using Fourier techniques ${ }^{2}$. The non-hydrogen atoms, with exceptions noted, were refined anisotropically and hydrogen atoms were treated as idealized contributions.

\section{REFERENCES}

(1) Z. Otwinowski and W. Minor, "Processing of X-Ray Diffraction Data Collected in Oscillation Mode," Methods in Enzymology, vol. 276: Macromolecular Crystallography, part A, 307-326, 1997, C.W. Carter, Jr. \& R.M. Sweet, Eds., Academic Press.

(2) Acta Cryst. A46 (1990) 467-473

\section{$\underline{\text { Structural Description }}$}

The molecule possesses a high degree of positional disorder in both the palladium complex and the co-crystallized solvent. Successful refinement in the space group $C 222_{1}$ yielded a composite of two orientations of the palladium complex with equal occupancy factors. Two alternative positions for the unsubstituted $\mathrm{Cp}$ ring, $\mathrm{C}(6-10)$, and the phenyl-ring, $\mathrm{C}(33-38)$, were modeled and refined with anisotropic displacement parameters. The palladium complex cocrystallized with one ordered molecule of tetrahydrofuran and one severely disordered molecule of pentane. The co-crystallized pentane resides in an infinitely extending channel formed by adjacent palladium complexes and propagating along the crystallographic $c$-axis. Thus the structure refinement represents an approximate location and orientation of the pentane molecule. All solvent atoms were refined isotropically. 
Table S14. Crystal data and structure refinement for $\mathbf{6}$.

Empirical formula

Formula weight

Temperature

Wavelength

Crystal system

Space group

Unit cell dimensions

Volume

$\mathrm{Z}$

Density (calculated)

Absorption coefficient

$\mathrm{F}(000)$

Crystal size

Theta range for data collection

Index ranges

Reflections collected

Independent reflections

Completeness to theta $=28.28^{\circ}$

Absorption correction

Max. and min. transmission

Refinement method

Data / restraints / parameters

Goodness-of-fit on $\mathrm{F}^{2}$

Final $\mathrm{R}$ indices [I $>2 \operatorname{sigma}(\mathrm{I})]$

$\mathrm{R}$ indices (all data)

Absolute structure parameter

Largest diff. peak and hole
$\mathrm{C}_{47} \mathrm{H}_{77} \mathrm{Br} \mathrm{Fe} \mathrm{O} \mathrm{P} 2 \mathrm{Pd}$

962.19

183(2) K

$0.71073 \AA$

Orthorhombic

C222(1)

$$
\begin{array}{ll}
\mathrm{a}=22.412(5) \AA & \alpha=90^{\circ} . \\
\mathrm{b}=24.106(5) \AA & \beta=90^{\circ} . \\
\mathrm{c}=16.388(3) \AA & \gamma=90^{\circ} .
\end{array}
$$$$
\text { 8854(3) } \AA^{3}
$$$$
8
$$

$1.444 \mathrm{~g} / \mathrm{cm}^{3}$

$17.43 \mathrm{~cm}^{-1}$

4032

$0.35 \times 0.20 \times 0.20 \mathrm{~mm}^{3}$

2.20 to $28.28^{\circ}$.

$-29<=\mathrm{h}<=29,-29<=\mathrm{k}<=31,-21<=\mathrm{l}<=21$

10177

10177 [R(int $)=0.0000]$

$99.1 \%$

None

0.7219 and 0.5806

Full-matrix least-squares on $\mathrm{F}^{2}$

10177 / 0 / 481

0.931

$\mathrm{R} 1=0.0481, \mathrm{wR} 2=0.1213$

$\mathrm{R} 1=0.0696, \mathrm{wR} 2=0.1361$

$0.013(10)$

0.599 and -0.732 e. $\AA^{-3}$ 
Table S15. Atomic coordinates ( $\mathrm{x} 10^{4}$ ) and equivalent isotropic displacement parameters $\left(\AA^{2} \mathrm{x}\right.$ $10^{3}$ )for 6 . $U(\mathrm{eq})$ is defined as one third of the trace of the orthogonalized $\mathrm{U}^{\mathrm{ij}}$ tensor.

\begin{tabular}{|c|c|c|c|c|}
\hline & $\mathrm{x}$ & $\mathrm{y}$ & $\mathrm{Z}$ & $\mathrm{U}(\mathrm{eq})$ \\
\hline $\operatorname{Pd}(1)$ & $2037(1)$ & $7075(1)$ & $4595(1)$ & $25(1)$ \\
\hline $\operatorname{Br}(1)$ & $2557(1)$ & $7212(1)$ & $3259(1)$ & $33(1)$ \\
\hline $\mathrm{Fe}(1)$ & $1506(1)$ & $5905(1)$ & $7230(1)$ & $32(1)$ \\
\hline $\mathrm{P}(2)$ & $2880(1)$ & $6733(1)$ & $5364(1)$ & $22(1)$ \\
\hline $\mathrm{P}(1)$ & $1297(1)$ & $6741(1)$ & $5418(1)$ & $26(1)$ \\
\hline $\mathrm{C}(1)$ & $1574(1)$ & $6164(1)$ & $6031(1)$ & $26(1)$ \\
\hline$C(2)$ & $2157(1)$ & $6126(1)$ & $6396(1)$ & $25(1)$ \\
\hline$C(3)$ & $2240(1)$ & $5579(1)$ & $6671(1)$ & $32(1)$ \\
\hline $\mathrm{C}(4)$ & $1725(1)$ & $5261(1)$ & $6482(1)$ & $33(1)$ \\
\hline$C(5)$ & 1313(1) & $5612(1)$ & $6086(1)$ & $31(1)$ \\
\hline$C(6)$ & $1005(1)$ & $6374(1)$ & 7961(1) & $33(1)$ \\
\hline$C(7)$ & $1605(1)$ & $6392(1)$ & $8239(1)$ & $26(1)$ \\
\hline $\mathrm{C}(8)$ & $1783(1)$ & $5842(1)$ & $8432(1)$ & $29(1)$ \\
\hline $\mathrm{C}(9)$ & $1294(1)$ & $5484(1)$ & $8273(1)$ & $33(1)$ \\
\hline$C(10)$ & $813(1)$ & $5813(1)$ & $7982(1)$ & $43(1)$ \\
\hline$C\left(6^{\prime}\right)$ & $727(1)$ & $6271(1)$ & $7805(1)$ & $45(1)$ \\
\hline$C\left(7^{\prime}\right)$ & $1255(1)$ & $6558(1)$ & $8035(1)$ & $49(1)$ \\
\hline $\mathrm{C}\left(8^{\prime}\right)$ & $1647(1)$ & $6170(1)$ & $8407(2)$ & $51(1)$ \\
\hline $\mathrm{C}\left(9^{\prime}\right)$ & $1362(1)$ & $5644(1)$ & $8406(2)$ & $47(1)$ \\
\hline$C\left(10^{\prime}\right)$ & 794(1) & $5706(1)$ & $8034(1)$ & $37(1)$ \\
\hline $\mathrm{C}(11)$ & $687(1)$ & $6441(1)$ & $4780(1)$ & $34(1)$ \\
\hline$C(12)$ & 131(1) & $6241(1)$ & $5219(1)$ & $44(1)$ \\
\hline $\mathrm{C}(13)$ & $-355(1)$ & 6093(1) & 4601(1) & $55(1)$ \\
\hline $\mathrm{C}(14)$ & $-123(1)$ & $5662(1)$ & $3984(1)$ & $74(1)$ \\
\hline $\mathrm{C}(15)$ & $446(1)$ & $5864(1)$ & $3555(1)$ & $61(1)$ \\
\hline$C(16)$ & $930(1)$ & $6019(1)$ & $4167(1)$ & $49(1)$ \\
\hline $\mathrm{C}(17)$ & $906(1)$ & $7231(1)$ & $6105(1)$ & $30(1)$ \\
\hline $\mathrm{C}(18)$ & 1371(1) & 7593(1) & $6557(1)$ & $46(1)$ \\
\hline $\mathrm{C}(19)$ & $1087(1)$ & $8024(1)$ & $7117(1)$ & $81(1)$ \\
\hline$C(20)$ & $638(1)$ & $8378(1)$ & $6646(2)$ & $95(1)$ \\
\hline
\end{tabular}




\begin{tabular}{|c|c|c|c|c|}
\hline$C(21)$ & $180(1)$ & $8033(1)$ & $6230(1)$ & $65(1)$ \\
\hline $\mathrm{C}(22)$ & $457(1)$ & $7609(1)$ & $5645(1)$ & $42(1)$ \\
\hline $\mathrm{C}(23)$ & $2615(1)$ & $6581(1)$ & $6433(1)$ & $25(1)$ \\
\hline $\mathrm{C}(24)$ & $3064(1)$ & $6478(1)$ & $7129(1)$ & $31(1)$ \\
\hline$C(25)$ & $3459(1)$ & $7299(1)$ & $5526(1)$ & $26(1)$ \\
\hline$C(26)$ & $3179(1)$ & $7729(1)$ & $6100(1)$ & $34(1)$ \\
\hline$C(27)$ & $4063(1)$ & $7099(1)$ & $5869(1)$ & $35(1)$ \\
\hline $\mathrm{C}(28)$ & $3586(1)$ & $7602(1)$ & $4716(1)$ & $31(1)$ \\
\hline$C(29)$ & $3229(1)$ & $6081(1)$ & $4920(1)$ & $26(1)$ \\
\hline $\mathrm{C}(30)$ & $3598(1)$ & $5743(1)$ & $5528(1)$ & $35(1)$ \\
\hline $\mathrm{C}(31)$ & $3631(1)$ & $6233(1)$ & 4201(1) & $35(1)$ \\
\hline $\mathrm{C}(32)$ & $2708(1)$ & $5736(1)$ & $4577(1)$ & $29(1)$ \\
\hline $\mathrm{C}(33)$ & $1425(1)$ & $7588(1)$ & 4081(1) & $65(1)$ \\
\hline C (35') & $631(1)$ & $8179(1)$ & $3296(1)$ & $66(1)$ \\
\hline C(37) & 894(1) & $8524(1)$ & $3737(1)$ & $82(1)$ \\
\hline $\mathrm{C}(34)$ & $973(1)$ & $7311(1)$ & $3397(1)$ & $25(1)$ \\
\hline$C(35)$ & $563(2)$ & $7686(2)$ & $3043(2)$ & $42(1)$ \\
\hline $\mathrm{C}(38)$ & $1360(1)$ & $8053(1)$ & $4198(2)$ & $30(1)$ \\
\hline C(34') & $1046(1)$ & $7601(1)$ & $3581(2)$ & $35(1)$ \\
\hline C(37') & $1309(2)$ & $8675(2)$ & 4199(2) & $45(1)$ \\
\hline $\mathrm{C}\left(38^{\prime}\right)$ & $1634(2)$ & $8215(1)$ & $4439(2)$ & $35(1)$ \\
\hline $\mathrm{O}(1)$ & 2092(2) & $4244(1)$ & $9345(2)$ & $180(1)$ \\
\hline C(39) & $2692(2)$ & $4178(2)$ & $8837(2)$ & $151(2)$ \\
\hline $\mathrm{C}(40)$ & $2479(2)$ & $4233(2)$ & $8031(2)$ & $157(2)$ \\
\hline $\mathrm{C}(41)$ & $1919(2)$ & $4057(2)$ & 7947(3) & $175(2)$ \\
\hline $\mathrm{C}(42)$ & $1689(1)$ & $4165(1)$ & $8653(1)$ & $73(1)$ \\
\hline C(43) & $4551(3)$ & $4969(4)$ & $7224(4)$ & $316(4)$ \\
\hline $\mathrm{C}(44)$ & $4123(3)$ & $4973(4)$ & $7949(3)$ & $283(3)$ \\
\hline$C(45)$ & 4707(3) & $5063(3)$ & $8718(4)$ & $238(3)$ \\
\hline$C(46)$ & $4457(4)$ & 4908(4) & $9494(3)$ & $377(6)$ \\
\hline $\mathrm{C}(47)$ & $4870(6)$ & 5000 & 10000 & $334(6)$ \\
\hline
\end{tabular}


Table S16. Bond lengths $[\AA]$ and angles $\left[{ }^{\circ}\right]$ for $\mathbf{6}$.

\begin{tabular}{|c|c|c|c|}
\hline $\operatorname{Pd}(1)-C(33)$ & 2.0291(19) & $\mathrm{C}\left(6^{\prime}\right)-\mathrm{C}\left(10^{\prime}\right)$ & 1.4200 \\
\hline $\mathrm{Pd}(1)-\mathrm{P}(1)$ & $2.2847(5)$ & $\mathrm{C}\left(7^{\prime}\right)-\mathrm{C}\left(8^{\prime}\right)$ & 1.4200 \\
\hline $\mathrm{Pd}(1)-\mathrm{P}(2)$ & $2.4157(5)$ & $\mathrm{C}\left(8^{\prime}\right)-\mathrm{C}\left(9^{\prime}\right)$ & 1.4200 \\
\hline $\operatorname{Pd}(1)-\operatorname{Br}(1)$ & $2.5031(4)$ & $\mathrm{C}\left(9^{\prime}\right)-\mathrm{C}\left(10^{\prime}\right)$ & 1.4200 \\
\hline $\mathrm{Fe}(1)-\mathrm{C}(6)$ & $1.9926(14)$ & $\mathrm{C}(11)-\mathrm{C}(12)$ & $1.517(2)$ \\
\hline $\mathrm{Fe}(1)-\mathrm{C}(10)$ & $1.9953(18)$ & $\mathrm{C}(11)-\mathrm{C}(16)$ & $1.531(2)$ \\
\hline $\mathrm{Fe}(1)-\mathrm{C}(4)$ & $2.0377(16)$ & $\mathrm{C}(12)-\mathrm{C}(13)$ & $1.528(2)$ \\
\hline $\mathrm{Fe}(1)-\mathrm{C}(7)$ & $2.0403(17)$ & $\mathrm{C}(13)-\mathrm{C}(14)$ & $1.540(3)$ \\
\hline $\mathrm{Fe}(1)-\mathrm{C}(3)$ & $2.0408(16)$ & $\mathrm{C}(14)-\mathrm{C}(15)$ & $1.535(3)$ \\
\hline $\mathrm{Fe}(1)-\mathrm{C}(9)$ & $2.045(2)$ & $\mathrm{C}(15)-\mathrm{C}(16)$ & $1.524(3)$ \\
\hline $\mathrm{Fe}(1)-\mathrm{C}(5)$ & $2.0498(15)$ & $\mathrm{C}(17)-\mathrm{C}(18)$ & $1.548(2)$ \\
\hline $\mathrm{Fe}(1)-\mathrm{C}\left(9^{\prime}\right)$ & $2.054(3)$ & $\mathrm{C}(17)-\mathrm{C}(22)$ & $1.552(2)$ \\
\hline $\mathrm{Fe}(1)-\mathrm{C}\left(8^{\prime}\right)$ & $2.057(3)$ & $\mathrm{C}(18)-\mathrm{C}(19)$ & $1.526(3)$ \\
\hline $\mathrm{Fe}(1)-\mathrm{C}(1)$ & $2.0671(13)$ & $\mathrm{C}(19)-\mathrm{C}(20)$ & $1.529(3)$ \\
\hline $\mathrm{Fe}(1)-\mathrm{C}(2)$ & $2.0691(14)$ & $\mathrm{C}(20)-\mathrm{C}(21)$ & $1.486(3)$ \\
\hline $\mathrm{Fe}(1)-\mathrm{C}(8)$ & $2.072(2)$ & $\mathrm{C}(21)-\mathrm{C}(22)$ & $1.533(3)$ \\
\hline$P(2)-C(23)$ & $1.8868(14)$ & $\mathrm{C}(23)-\mathrm{C}(24)$ & $1.543(2)$ \\
\hline $\mathrm{P}(2)-\mathrm{C}(25)$ & $1.9004(15)$ & $C(25)-C(26)$ & $1.536(2)$ \\
\hline $\mathrm{P}(2)-\mathrm{C}(29)$ & $1.9008(15)$ & $\mathrm{C}(25)-\mathrm{C}(28)$ & $1.5418(19)$ \\
\hline $\mathrm{P}(1)-\mathrm{C}(1)$ & $1.8236(15)$ & $\mathrm{C}(25)-\mathrm{C}(27)$ & $1.544(2)$ \\
\hline $\mathrm{P}(1)-\mathrm{C}(17)$ & $1.8525(15)$ & $\mathrm{C}(29)-\mathrm{C}(31)$ & $1.527(2)$ \\
\hline $\mathrm{P}(1)-\mathrm{C}(11)$ & $1.8658(16)$ & $\mathrm{C}(29)-\mathrm{C}(30)$ & $1.529(2)$ \\
\hline $\mathrm{C}(1)-\mathrm{C}(2)$ & $1.440(2)$ & $\mathrm{C}(29)-\mathrm{C}(32)$ & $1.541(2)$ \\
\hline $\mathrm{C}(1)-\mathrm{C}(5)$ & $1.458(2)$ & $\mathrm{C}(33)-\mathrm{C}(38)$ & $1.147(3)$ \\
\hline$C(2)-C(3)$ & $1.406(2)$ & $\mathrm{C}(33)-\mathrm{C}\left(34^{\prime}\right)$ & $1.182(3)$ \\
\hline$C(2)-C(23)$ & $1.502(2)$ & $\mathrm{C}(33)-\mathrm{C}(34)$ & $1.653(3)$ \\
\hline$C(3)-C(4)$ & $1.421(2)$ & $\mathrm{C}(33)-\mathrm{C}\left(38^{\prime}\right)$ & $1.687(4)$ \\
\hline$C(4)-C(5)$ & $1.410(2)$ & $\mathrm{C}\left(35^{\prime}\right)-\mathrm{C}(37)$ & $1.250(3)$ \\
\hline$C(6)-C(7)$ & 1.4200 & $\mathrm{C}\left(35^{\prime}\right)-\mathrm{C}(35)$ & $1.269(4)$ \\
\hline$C(6)-C(10)$ & 1.4200 & $\mathrm{C}\left(35^{\prime}\right)-\mathrm{C}\left(34^{\prime}\right)$ & $1.741(4)$ \\
\hline$C(7)-C(8)$ & 1.4200 & $\mathrm{C}(37)-\mathrm{C}\left(37^{\prime}\right)$ & $1.252(4)$ \\
\hline $\mathrm{C}(8)-\mathrm{C}(9)$ & 1.4200 & $\mathrm{C}(37)-\mathrm{C}(38)$ & $1.717(4)$ \\
\hline $\mathrm{C}(9)-\mathrm{C}(10)$ & 1.4200 & $\mathrm{C}(34)-\mathrm{C}(35)$ & $1.414(4)$ \\
\hline $\mathrm{C}\left(6^{\prime}\right)-\mathrm{C}\left(7^{\prime}\right)$ & 1.4200 & $\mathrm{C}\left(37^{\prime}\right)-\mathrm{C}\left(38^{\prime}\right)$ & $1.383(5)$ \\
\hline
\end{tabular}




\begin{tabular}{|c|c|c|c|}
\hline $\mathrm{O}(1)-\mathrm{C}(42)$ & $1.461(4)$ & $\mathrm{C}(7)-\mathrm{Fe}(1)-\mathrm{C}(5)$ & $164.38(6)$ \\
\hline $\mathrm{O}(1)-\mathrm{C}(39)$ & $1.590(6)$ & $\mathrm{C}(3)-\mathrm{Fe}(1)-\mathrm{C}(5)$ & $68.10(6)$ \\
\hline C(39)-C(40) & $1.410(5)$ & $\mathrm{C}(9)-\mathrm{Fe}(1)-\mathrm{C}(5)$ & $122.99(8)$ \\
\hline $\mathrm{C}(40)-\mathrm{C}(41)$ & $1.332(7)$ & $\mathrm{C}(6)-\mathrm{Fe}(1)-\mathrm{C}\left(9^{\prime}\right)$ & $61.38(8)$ \\
\hline$C(41)-C(42)$ & $1.294(5)$ & $\mathrm{C}(10)-\mathrm{Fe}(1)-\mathrm{C}\left(9^{\prime}\right)$ & $42.56(8)$ \\
\hline C(43)-C(44) & $1.526(9)$ & $\mathrm{C}(4)-\mathrm{Fe}(1)-\mathrm{C}\left(9^{\prime}\right)$ & $111.59(9)$ \\
\hline$C(44)-C(45)$ & $1.829(9)$ & $\mathrm{C}(7)-\mathrm{Fe}(1)-\mathrm{C}\left(9^{\prime}\right)$ & $55.43(10)$ \\
\hline$C(45)-C(46)$ & $1.439(9)$ & $\mathrm{C}(3)-\mathrm{Fe}(1)-\mathrm{C}\left(9^{\prime}\right)$ & $115.40(8)$ \\
\hline$C(46)-C(47)$ & $1.261(13)$ & $\mathrm{C}(9)-\mathrm{Fe}(1)-\mathrm{C}\left(9^{\prime}\right)$ & $13.10(10)$ \\
\hline C(46)-C(46)\#1 & $1.717(12)$ & $\mathrm{C}(5)-\mathrm{Fe}(1)-\mathrm{C}\left(9^{\prime}\right)$ & $136.00(10)$ \\
\hline \multirow[t]{2}{*}{$C(47)-C(46) \# 1$} & $1.261(13)$ & $\mathrm{C}(6)-\mathrm{Fe}(1)-\mathrm{C}\left(8^{\prime}\right)$ & $49.20(7)$ \\
\hline & & $\mathrm{C}(10)-\mathrm{Fe}(1)-\mathrm{C}\left(8^{\prime}\right)$ & $64.82(8)$ \\
\hline $\mathrm{C}(33)-\mathrm{Pd}(1)-\mathrm{P}(1)$ & $88.27(5)$ & $\mathrm{C}(4)-\mathrm{Fe}(1)-\mathrm{C}\left(8^{\prime}\right)$ & 139.74(9) \\
\hline $\mathrm{C}(33)-\mathrm{Pd}(1)-\mathrm{P}(2)$ & $162.27(6)$ & $\mathrm{C}(7)-\mathrm{Fe}(1)-\mathrm{C}\left(8^{\prime}\right)$ & 17.11(9) \\
\hline $\mathrm{P}(1)-\mathrm{Pd}(1)-\mathrm{P}(2)$ & $98.045(19)$ & $\mathrm{C}(3)-\mathrm{Fe}(1)-\mathrm{C}\left(8^{\prime}\right)$ & $114.57(8)$ \\
\hline$C(33)-\operatorname{Pd}(1)-\operatorname{Br}(1)$ & $82.61(5)$ & $\mathrm{C}(9)-\mathrm{Fe}(1)-\mathrm{C}\left(8^{\prime}\right)$ & $53.48(10)$ \\
\hline $\mathrm{P}(1)-\mathrm{Pd}(1)-\mathrm{Br}(1)$ & $154.346(11)$ & $\mathrm{C}(5)-\mathrm{Fe}(1)-\mathrm{C}\left(8^{\prime}\right)$ & $175.93(9)$ \\
\hline $\mathrm{P}(2)-\mathrm{Pd}(1)-\mathrm{Br}(1)$ & $97.833(16)$ & $\mathrm{C}\left(9^{\prime}\right)-\mathrm{Fe}(1)-\mathrm{C}\left(8^{\prime}\right)$ & $40.41(5)$ \\
\hline $\mathrm{C}(6)-\mathrm{Fe}(1)-\mathrm{C}(10)$ & 41.7 & $\mathrm{C}(6)-\mathrm{Fe}(1)-\mathrm{C}(1)$ & $116.26(6)$ \\
\hline $\mathrm{C}(6)-\mathrm{Fe}(1)-\mathrm{C}(4)$ & $158.28(6)$ & $\mathrm{C}(10)-\mathrm{Fe}(1)-\mathrm{C}(1)$ & $132.76(7)$ \\
\hline $\mathrm{C}(10)-\mathrm{Fe}(1)-\mathrm{C}(4)$ & $118.26(6)$ & $\mathrm{C}(4)-\mathrm{Fe}(1)-\mathrm{C}(1)$ & $68.96(6)$ \\
\hline $\mathrm{C}(6)-\mathrm{Fe}(1)-\mathrm{C}(7)$ & 41.2 & $\mathrm{C}(7)-\mathrm{Fe}(1)-\mathrm{C}(1)$ & $126.07(7)$ \\
\hline $\mathrm{C}(10)-\mathrm{Fe}(1)-\mathrm{C}(7)$ & 69.4 & $\mathrm{C}(3)-\mathrm{Fe}(1)-\mathrm{C}(1)$ & $68.31(6)$ \\
\hline $\mathrm{C}(4)-\mathrm{Fe}(1)-\mathrm{C}(7)$ & $154.08(6)$ & $\mathrm{C}(9)-\mathrm{Fe}(1)-\mathrm{C}(1)$ & $164.17(7)$ \\
\hline $\mathrm{C}(6)-\mathrm{Fe}(1)-\mathrm{C}(3)$ & $160.41(6)$ & $\mathrm{C}(5)-\mathrm{Fe}(1)-\mathrm{C}(1)$ & $41.47(6)$ \\
\hline $\mathrm{C}(10)-\mathrm{Fe}(1)-\mathrm{C}(3)$ & $149.49(6)$ & $\mathrm{C}\left(9^{\prime}\right)-\mathrm{Fe}(1)-\mathrm{C}(1)$ & $175.21(8)$ \\
\hline $\mathrm{C}(4)-\mathrm{Fe}(1)-\mathrm{C}(3)$ & $40.78(6)$ & $\mathrm{C}\left(8^{\prime}\right)-\mathrm{Fe}(1)-\mathrm{C}(1)$ & $141.88(10)$ \\
\hline $\mathrm{C}(7)-\mathrm{Fe}(1)-\mathrm{C}(3)$ & $119.80(6)$ & $\mathrm{C}(6)-\mathrm{Fe}(1)-\mathrm{C}(2)$ & $130.42(6)$ \\
\hline $\mathrm{C}(6)-\mathrm{Fe}(1)-\mathrm{C}(9)$ & $69.36(5)$ & $\mathrm{C}(10)-\mathrm{Fe}(1)-\mathrm{C}(2)$ & $170.36(6)$ \\
\hline $\mathrm{C}(10)-\mathrm{Fe}(1)-\mathrm{C}(9)$ & 41.1 & $\mathrm{C}(4)-\mathrm{Fe}(1)-\mathrm{C}(2)$ & $68.26(6)$ \\
\hline $\mathrm{C}(4)-\mathrm{Fe}(1)-\mathrm{C}(9)$ & $100.38(7)$ & $\mathrm{C}(7)-\mathrm{Fe}(1)-\mathrm{C}(2)$ & $108.06(6)$ \\
\hline $\mathrm{C}(7)-\mathrm{Fe}(1)-\mathrm{C}(9)$ & $68.45(6)$ & $\mathrm{C}(3)-\mathrm{Fe}(1)-\mathrm{C}(2)$ & $40.02(6)$ \\
\hline $\mathrm{C}(3)-\mathrm{Fe}(1)-\mathrm{C}(9)$ & $111.77(7)$ & $\mathrm{C}(9)-\mathrm{Fe}(1)-\mathrm{C}(2)$ & $147.54(7)$ \\
\hline $\mathrm{C}(6)-\mathrm{Fe}(1)-\mathrm{C}(5)$ & $128.85(6)$ & $\mathrm{C}(5)-\mathrm{Fe}(1)-\mathrm{C}(2)$ & $68.53(6)$ \\
\hline $\mathrm{C}(10)-\mathrm{Fe}(1)-\mathrm{C}(5)$ & $111.26(7)$ & $\mathrm{C}\left(9^{\prime}\right)-\mathrm{Fe}(1)-\mathrm{C}(2)$ & $144.03(8)$ \\
\hline $\mathrm{C}(4)-\mathrm{Fe}(1)-\mathrm{C}(5)$ & $40.34(6)$ & $\mathrm{C}\left(8^{\prime}\right)-\mathrm{Fe}(1)-\mathrm{C}(2)$ & $115.52(8)$ \\
\hline
\end{tabular}




\begin{tabular}{|c|c|c|c|}
\hline $\mathrm{C}(1)-\mathrm{Fe}(1)-\mathrm{C}(2)$ & $40.76(6)$ & $\mathrm{C}(2)-\mathrm{C}(3)-\mathrm{C}(4)$ & $109.18(13)$ \\
\hline $\mathrm{C}(6)-\mathrm{Fe}(1)-\mathrm{C}(8)$ & 68.8 & $\mathrm{C}(2)-\mathrm{C}(3)-\mathrm{Fe}(1)$ & $71.08(9)$ \\
\hline $\mathrm{C}(10)-\mathrm{Fe}(1)-\mathrm{C}(8)$ & $68.76(6)$ & $\mathrm{C}(4)-\mathrm{C}(3)-\mathrm{Fe}(1)$ & $69.49(9)$ \\
\hline $\mathrm{C}(4)-\mathrm{Fe}(1)-\mathrm{C}(8)$ & $116.32(7)$ & $C(5)-C(4)-C(3)$ & $108.02(14)$ \\
\hline $\mathrm{C}(7)-\mathrm{Fe}(1)-\mathrm{C}(8)$ & 40.4 & $\mathrm{C}(5)-\mathrm{C}(4)-\mathrm{Fe}(1)$ & $70.29(9)$ \\
\hline $\mathrm{C}(3)-\mathrm{Fe}(1)-\mathrm{C}(8)$ & $98.96(7)$ & $\mathrm{C}(3)-\mathrm{C}(4)-\mathrm{Fe}(1)$ & $69.73(9)$ \\
\hline $\mathrm{C}(9)-\mathrm{Fe}(1)-\mathrm{C}(8)$ & 40.4 & $\mathrm{C}(4)-\mathrm{C}(5)-\mathrm{C}(1)$ & $108.27(13)$ \\
\hline $\mathrm{C}(5)-\mathrm{Fe}(1)-\mathrm{C}(8)$ & $155.18(7)$ & $\mathrm{C}(4)-\mathrm{C}(5)-\mathrm{Fe}(1)$ & $69.36(9)$ \\
\hline $\mathrm{C}\left(9^{\prime}\right)-\mathrm{Fe}(1)-\mathrm{C}(8)$ & $29.76(10)$ & $\mathrm{C}(1)-\mathrm{C}(5)-\mathrm{Fe}(1)$ & $69.90(8)$ \\
\hline $\mathrm{C}\left(8^{\prime}\right)-\mathrm{Fe}(1)-\mathrm{C}(8)$ & $23.69(9)$ & $\mathrm{C}(7)-\mathrm{C}(6)-\mathrm{C}(10)$ & 108.0 \\
\hline $\mathrm{C}(1)-\mathrm{Fe}(1)-\mathrm{C}(8)$ & $154.65(8)$ & $\mathrm{C}(7)-\mathrm{C}(6)-\mathrm{Fe}(1)$ & $71.20(7)$ \\
\hline $\mathrm{C}(2)-\mathrm{Fe}(1)-\mathrm{C}(8)$ & $115.76(7)$ & $\mathrm{C}(10)-\mathrm{C}(6)-\mathrm{Fe}(1)$ & $69.24(6)$ \\
\hline $\mathrm{C}(23)-\mathrm{P}(2)-\mathrm{C}(25)$ & $102.98(6)$ & $\mathrm{C}(8)-\mathrm{C}(7)-\mathrm{C}(6)$ & 108.0 \\
\hline $\mathrm{C}(23)-\mathrm{P}(2)-\mathrm{C}(29)$ & $108.92(6)$ & $\mathrm{C}(8)-\mathrm{C}(7)-\mathrm{Fe}(1)$ & $71.00(6)$ \\
\hline $\mathrm{C}(25)-\mathrm{P}(2)-\mathrm{C}(29)$ & $111.42(6)$ & $\mathrm{C}(6)-\mathrm{C}(7)-\mathrm{Fe}(1)$ & $67.59(6)$ \\
\hline $\mathrm{C}(23)-\mathrm{P}(2)-\mathrm{Pd}(1)$ & $107.71(5)$ & $\mathrm{C}(9)-\mathrm{C}(8)-\mathrm{C}(7)$ & 108.0 \\
\hline $\mathrm{C}(25)-\mathrm{P}(2)-\mathrm{Pd}(1)$ & $111.25(5)$ & $\mathrm{C}(9)-\mathrm{C}(8)-\mathrm{Fe}(1)$ & $68.79(6)$ \\
\hline C(29)-P(2)-Pd(1) & $113.90(5)$ & $\mathrm{C}(7)-\mathrm{C}(8)-\mathrm{Fe}(1)$ & 68.6 \\
\hline $\mathrm{C}(1)-\mathrm{P}(1)-\mathrm{C}(17)$ & $108.21(7)$ & $\mathrm{C}(10)-\mathrm{C}(9)-\mathrm{C}(8)$ & 108.0 \\
\hline $\mathrm{C}(1)-\mathrm{P}(1)-\mathrm{C}(11)$ & $105.29(7)$ & $\mathrm{C}(10)-\mathrm{C}(9)-\mathrm{Fe}(1)$ & $67.57(6)$ \\
\hline $\mathrm{C}(17)-\mathrm{P}(1)-\mathrm{C}(11)$ & $103.95(7)$ & $\mathrm{C}(8)-\mathrm{C}(9)-\mathrm{Fe}(1)$ & $70.86(6)$ \\
\hline $\mathrm{C}(1)-\mathrm{P}(1)-\mathrm{Pd}(1)$ & $110.25(5)$ & $\mathrm{C}(9)-\mathrm{C}(10)-\mathrm{C}(6)$ & 108.0 \\
\hline $\mathrm{C}(17)-\mathrm{P}(1)-\mathrm{Pd}(1)$ & $118.48(5)$ & $\mathrm{C}(9)-\mathrm{C}(10)-\mathrm{Fe}(1)$ & $71.29(6)$ \\
\hline $\mathrm{C}(11)-\mathrm{P}(1)-\mathrm{Pd}(1)$ & $109.74(5)$ & $\mathrm{C}(6)-\mathrm{C}(10)-\mathrm{Fe}(1)$ & $69.04(5)$ \\
\hline $\mathrm{C}(2)-\mathrm{C}(1)-\mathrm{C}(5)$ & $106.30(12)$ & $\mathrm{C}\left(7^{\prime}\right)-\mathrm{C}\left(6^{\prime}\right)-\mathrm{C}\left(10^{\prime}\right)$ & 108.0 \\
\hline $\mathrm{C}(2)-\mathrm{C}(1)-\mathrm{P}(1)$ & $125.92(11)$ & $\mathrm{C}\left(7^{\prime}\right)-\mathrm{C}\left(6^{\prime}\right)-\mathrm{Fe}(1)$ & $69.14(8)$ \\
\hline $\mathrm{C}(5)-\mathrm{C}(1)-\mathrm{P}(1)$ & $126.39(11)$ & $\mathrm{C}\left(10^{\prime}\right)-\mathrm{C}\left(6^{\prime}\right)-\mathrm{Fe}(1)$ & $69.00(8)$ \\
\hline $\mathrm{C}(2)-\mathrm{C}(1)-\mathrm{Fe}(1)$ & $69.70(7)$ & $\mathrm{C}\left(8^{\prime}\right)-\mathrm{C}\left(7^{\prime}\right)-\mathrm{C}\left(6^{\prime}\right)$ & 108.0 \\
\hline $\mathrm{C}(5)-\mathrm{C}(1)-\mathrm{Fe}(1)$ & $68.63(8)$ & $\mathrm{C}\left(8^{\prime}\right)-\mathrm{C}\left(7^{\prime}\right)-\mathrm{Fe}(1)$ & $67.45(8)$ \\
\hline $\mathrm{P}(1)-\mathrm{C}(1)-\mathrm{Fe}(1)$ & $136.73(8)$ & $\mathrm{C}\left(6^{\prime}\right)-\mathrm{C}\left(7^{\prime}\right)-\mathrm{Fe}(1)$ & $72.30(7)$ \\
\hline $\mathrm{C}(3)-\mathrm{C}(2)-\mathrm{C}(1)$ & $108.22(13)$ & $\mathrm{C}\left(7^{\prime}\right)-\mathrm{C}\left(8^{\prime}\right)-\mathrm{C}\left(9^{\prime}\right)$ & 108.0 \\
\hline $\mathrm{C}(3)-\mathrm{C}(2)-\mathrm{C}(23)$ & $125.54(13)$ & $\mathrm{C}\left(7^{\prime}\right)-\mathrm{C}\left(8^{\prime}\right)-\mathrm{Fe}(1)$ & 72.93(7) \\
\hline $\mathrm{C}(1)-\mathrm{C}(2)-\mathrm{C}(23)$ & $126.14(13)$ & $\mathrm{C}\left(9^{\prime}\right)-\mathrm{C}\left(8^{\prime}\right)-\mathrm{Fe}(1)$ & $69.71(9)$ \\
\hline $\mathrm{C}(3)-\mathrm{C}(2)-\mathrm{Fe}(1)$ & $68.91(8)$ & $\mathrm{C}\left(10^{\prime}\right)-\mathrm{C}\left(9^{\prime}\right)-\mathrm{C}\left(8^{\prime}\right)$ & 108.0 \\
\hline $\mathrm{C}(1)-\mathrm{C}(2)-\mathrm{Fe}(1)$ & $69.55(8)$ & $\mathrm{C}\left(10^{\prime}\right)-\mathrm{C}\left(9^{\prime}\right)-\mathrm{Fe}(1)$ & $72.86(7)$ \\
\hline $\mathrm{C}(23)-\mathrm{C}(2)-\mathrm{Fe}(1)$ & $130.02(10)$ & $\mathrm{C}\left(8^{\prime}\right)-\mathrm{C}\left(9^{\prime}\right)-\mathrm{Fe}(1)$ & $69.87(8)$ \\
\hline
\end{tabular}




\begin{tabular}{|c|c|c|c|}
\hline $\mathrm{C}\left(9^{\prime}\right)-\mathrm{C}\left(10^{\prime}\right)-\mathrm{C}\left(6^{\prime}\right)$ & 108.0 & $\mathrm{C}\left(34^{\prime}\right)-\mathrm{C}(33)-\mathrm{C}(34)$ & $25.6(2)$ \\
\hline $\mathrm{C}\left(9^{\prime}\right)-\mathrm{C}\left(10^{\prime}\right)-\mathrm{Fe}(1)$ & $67.47(8)$ & $\mathrm{C}(38)-\mathrm{C}(33)-\mathrm{C}\left(38^{\prime}\right)$ & $26.07(19)$ \\
\hline $\mathrm{C}\left(6^{\prime}\right)-\mathrm{C}\left(10^{\prime}\right)-\mathrm{Fe}(1)$ & $72.41(7)$ & $\mathrm{C}\left(34^{\prime}\right)-\mathrm{C}(33)-\mathrm{C}\left(38^{\prime}\right)$ & $114.7(2)$ \\
\hline$C(12)-C(11)-C(16)$ & $113.13(14)$ & $\mathrm{C}(34)-\mathrm{C}(33)-\mathrm{C}\left(38^{\prime}\right)$ & $140.21(19)$ \\
\hline $\mathrm{C}(12)-\mathrm{C}(11)-\mathrm{P}(1)$ & $117.32(11)$ & $\mathrm{C}(38)-\mathrm{C}(33)-\mathrm{Pd}(1)$ & $127.8(2)$ \\
\hline $\mathrm{C}(16)-\mathrm{C}(11)-\mathrm{P}(1)$ & $111.34(11)$ & $\mathrm{C}\left(34^{\prime}\right)-\mathrm{C}(33)-\mathrm{Pd}(1)$ & $142.1(2)$ \\
\hline$C(11)-C(12)-C(13)$ & $110.14(14)$ & $\mathrm{C}(34)-\mathrm{C}(33)-\mathrm{Pd}(1)$ & $116.66(15)$ \\
\hline$C(12)-C(13)-C(14)$ & $110.67(15)$ & $\mathrm{C}\left(38^{\prime}\right)-\mathrm{C}(33)-\mathrm{Pd}(1)$ & $102.40(14)$ \\
\hline $\mathrm{C}(15)-\mathrm{C}(14)-\mathrm{C}(13)$ & $111.55(17)$ & $\mathrm{C}(37)-\mathrm{C}\left(35^{\prime}\right)-\mathrm{C}(35)$ & $150.3(3)$ \\
\hline$C(16)-C(15)-C(14)$ & $111.64(15)$ & $\mathrm{C}(37)-\mathrm{C}\left(35^{\prime}\right)-\mathrm{C}\left(34^{\prime}\right)$ & $97.2(2)$ \\
\hline$C(15)-C(16)-C(11)$ & $109.96(15)$ & $\mathrm{C}(35)-\mathrm{C}\left(35^{\prime}\right)-\mathrm{C}\left(34^{\prime}\right)$ & $53.1(2)$ \\
\hline$C(18)-C(17)-C(22)$ & $109.70(14)$ & $\mathrm{C}\left(35^{\prime}\right)-\mathrm{C}(37)-\mathrm{C}\left(37^{\prime}\right)$ & $153.2(3)$ \\
\hline $\mathrm{C}(18)-\mathrm{C}(17)-\mathrm{P}(1)$ & $109.42(10)$ & $\mathrm{C}\left(35^{\prime}\right)-\mathrm{C}(37)-\mathrm{C}(38)$ & $95.9(2)$ \\
\hline $\mathrm{C}(22)-\mathrm{C}(17)-\mathrm{P}(1)$ & $112.64(10)$ & $\mathrm{C}\left(37^{\prime}\right)-\mathrm{C}(37)-\mathrm{C}(38)$ & $58.3(2)$ \\
\hline $\mathrm{C}(19)-\mathrm{C}(18)-\mathrm{C}(17)$ & $113.03(14)$ & $\mathrm{C}(35)-\mathrm{C}(34)-\mathrm{C}(33)$ & $114.7(2)$ \\
\hline$C(18)-C(19)-C(20)$ & $110.47(16)$ & $\mathrm{C}\left(35^{\prime}\right)-\mathrm{C}(35)-\mathrm{C}(34)$ & $112.9(3)$ \\
\hline$C(21)-C(20)-C(19)$ & $111.93(18)$ & $\mathrm{C}(33)-\mathrm{C}(38)-\mathrm{C}(37)$ & $130.6(3)$ \\
\hline$C(20)-C(21)-C(22)$ & $112.33(16)$ & $\mathrm{C}(33)-\mathrm{C}\left(34^{\prime}\right)-\mathrm{C}\left(35^{\prime}\right)$ & $126.2(3)$ \\
\hline $\mathrm{C}(21)-\mathrm{C}(22)-\mathrm{C}(17)$ & $110.47(13)$ & $\mathrm{C}(37)-\mathrm{C}\left(37^{\prime}\right)-\mathrm{C}\left(38^{\prime}\right)$ & $109.3(3)$ \\
\hline $\mathrm{C}(2)-\mathrm{C}(23)-\mathrm{C}(24)$ & $111.01(12)$ & $\mathrm{C}\left(37^{\prime}\right)-\mathrm{C}\left(38^{\prime}\right)-\mathrm{C}(33)$ & 118.2(3) \\
\hline $\mathrm{C}(2)-\mathrm{C}(23)-\mathrm{P}(2)$ & $108.63(9)$ & $\mathrm{C}(42)-\mathrm{O}(1)-\mathrm{C}(39)$ & $96.0(2)$ \\
\hline $\mathrm{C}(24)-\mathrm{C}(23)-\mathrm{P}(2)$ & $120.84(10)$ & $\mathrm{C}(40)-\mathrm{C}(39)-\mathrm{O}(1)$ & 101.2(4) \\
\hline$C(26)-C(25)-C(28)$ & $106.45(12)$ & $\mathrm{C}(41)-\mathrm{C}(40)-\mathrm{C}(39)$ & $112.7(4)$ \\
\hline$C(26)-C(25)-C(27)$ & $110.23(12)$ & $\mathrm{C}(42)-\mathrm{C}(41)-\mathrm{C}(40)$ & $102.6(4)$ \\
\hline$C(28)-C(25)-C(27)$ & $107.42(12)$ & $\mathrm{C}(41)-\mathrm{C}(42)-\mathrm{O}(1)$ & $118.3(3)$ \\
\hline $\mathrm{C}(26)-\mathrm{C}(25)-\mathrm{P}(2)$ & $106.94(10)$ & $\mathrm{C}(43)-\mathrm{C}(44)-\mathrm{C}(45)$ & $95.1(5)$ \\
\hline $\mathrm{C}(28)-\mathrm{C}(25)-\mathrm{P}(2)$ & $110.21(9)$ & $\mathrm{C}(46)-\mathrm{C}(45)-\mathrm{C}(44)$ & $107.5(6)$ \\
\hline $\mathrm{C}(27)-\mathrm{C}(25)-\mathrm{P}(2)$ & $115.26(10)$ & $\mathrm{C}(47)-\mathrm{C}(46)-\mathrm{C}(45)$ & $104.5(8)$ \\
\hline $\mathrm{C}(31)-\mathrm{C}(29)-\mathrm{C}(30)$ & $108.16(12)$ & $\mathrm{C}(47)-\mathrm{C}(46)-\mathrm{C}(46) \# 1$ & $47.1(6)$ \\
\hline $\mathrm{C}(31)-\mathrm{C}(29)-\mathrm{C}(32)$ & $107.12(12)$ & $\mathrm{C}(45)-\mathrm{C}(46)-\mathrm{C}(46) \# 1$ & $141.9(9)$ \\
\hline $\mathrm{C}(30)-\mathrm{C}(29)-\mathrm{C}(32)$ & $111.08(12)$ & $\mathrm{C}(46) \# 1-\mathrm{C}(47)-\mathrm{C}(46)$ & $85.8(12)$ \\
\hline $\mathrm{C}(31)-\mathrm{C}(29)-\mathrm{P}(2)$ & $109.84(10)$ & $\mathrm{C}(38)-\mathrm{C}(33)-\mathrm{C}(34)$ & $115.5(2)$ \\
\hline $\mathrm{C}(30)-\mathrm{C}(29)-\mathrm{P}(2)$ & $114.51(10)$ & $\mathrm{C}(38)-\mathrm{C}(33)-\mathrm{C}\left(34^{\prime}\right)$ & $90.0(3)$ \\
\hline $\mathrm{C}(32)-\mathrm{C}(29)-\mathrm{P}(2)$ & $105.89(10)$ & & \\
\hline
\end{tabular}

Symmetry transformations used to generate equivalent atoms: $\# 1 \mathrm{x},-\mathrm{y}+1,-\mathrm{z}+2$ 
Table S17. Anisotropic displacement parameters $\left(\AA^{2} \times 10^{3}\right)$ for 6 . The anisotropic displacement factor exponent takes the form: $-2 \pi^{2}\left[h^{2} a^{* 2} U^{11}+\ldots+2 h k a^{*} b^{*} U^{12}\right]$

\begin{tabular}{|c|c|c|c|c|c|c|}
\hline & $\mathrm{U}^{11}$ & $\mathrm{U}^{22}$ & $\mathrm{U}^{33}$ & $\mathrm{U}^{23}$ & $\mathrm{U}^{13}$ & $\mathrm{U}^{12}$ \\
\hline $\operatorname{Pd}(1)$ & $27(1)$ & $30(1)$ & $18(1)$ & $0(1)$ & $1(1)$ & $7(1)$ \\
\hline $\operatorname{Br}(1)$ & $32(1)$ & $45(1)$ & $22(1)$ & $5(1)$ & $3(1)$ & $2(1)$ \\
\hline $\mathrm{Fe}(1)$ & $35(1)$ & $37(1)$ & $24(1)$ & $1(1)$ & $2(1)$ & $-14(1)$ \\
\hline $\mathrm{P}(2)$ & $22(1)$ & $23(1)$ & $20(1)$ & $2(1)$ & $-2(1)$ & $-3(1)$ \\
\hline $\mathrm{P}(1)$ & $21(1)$ & $37(1)$ & $20(1)$ & $-4(1)$ & $0(1)$ & $5(1)$ \\
\hline$C(1)$ & $25(1)$ & $35(1)$ & 19(1) & $-1(1)$ & $-2(1)$ & $1(1)$ \\
\hline$C(2)$ & $22(1)$ & $32(1)$ & 21(1) & $5(1)$ & $-1(1)$ & $-3(1)$ \\
\hline $\mathrm{C}(3)$ & $31(1)$ & $33(1)$ & $32(1)$ & $6(1)$ & $-2(1)$ & $-2(1)$ \\
\hline$C(4)$ & $35(1)$ & $25(1)$ & $39(1)$ & $-3(1)$ & $-3(1)$ & $-9(1)$ \\
\hline$C(5)$ & $28(1)$ & $37(1)$ & $28(1)$ & $-9(1)$ & $3(1)$ & $-8(1)$ \\
\hline$C(6)$ & $26(1)$ & $61(2)$ & 11(1) & $-3(1)$ & $5(1)$ & $10(2)$ \\
\hline$C(7)$ & $41(2)$ & $12(1)$ & $25(1)$ & $-4(1)$ & $7(1)$ & $1(1)$ \\
\hline $\mathrm{C}(8)$ & $50(2)$ & $22(1)$ & $15(1)$ & $5(1)$ & $-5(1)$ & $-6(1)$ \\
\hline $\mathrm{C}(9)$ & $41(2)$ & $33(2)$ & $24(1)$ & $14(1)$ & $12(1)$ & $-11(1)$ \\
\hline $\mathrm{C}(10)$ & $39(2)$ & $38(2)$ & $51(2)$ & $5(1)$ & $33(1)$ & $-21(1)$ \\
\hline$C\left(6^{\prime}\right)$ & $45(2)$ & $45(2)$ & $43(2)$ & $1(2)$ & $1(2)$ & $-2(2)$ \\
\hline$C\left(7^{\prime}\right)$ & $51(2)$ & $50(2)$ & $46(2)$ & $-1(2)$ & $4(2)$ & $-6(2)$ \\
\hline$C\left(8^{\prime}\right)$ & $52(2)$ & $52(2)$ & $49(2)$ & $0(2)$ & $0(2)$ & $-1(2)$ \\
\hline $\mathrm{C}\left(9^{\prime}\right)$ & $49(2)$ & $48(2)$ & $43(2)$ & $5(2)$ & $3(2)$ & $1(2)$ \\
\hline $\mathrm{C}\left(10^{\prime}\right)$ & $40(2)$ & $34(2)$ & $37(2)$ & $1(1)$ & $0(2)$ & $1(2)$ \\
\hline $\mathrm{C}(11)$ & $31(1)$ & $43(1)$ & $28(1)$ & $-8(1)$ & $-12(1)$ & $8(1)$ \\
\hline$C(12)$ & $26(1)$ & $58(1)$ & $47(1)$ & $-13(1)$ & $-13(1)$ & $-2(1)$ \\
\hline$C(13)$ & $34(1)$ & $63(1)$ & $70(1)$ & $-24(1)$ & $-24(1)$ & $4(1)$ \\
\hline $\mathrm{C}(14)$ & $55(1)$ & $76(1)$ & $92(1)$ & $-39(1)$ & $-45(1)$ & $17(1)$ \\
\hline$C(15)$ & $54(1)$ & $73(1)$ & $55(1)$ & $-39(1)$ & $-35(1)$ & $25(1)$ \\
\hline$C(16)$ & $47(1)$ & $60(1)$ & $39(1)$ & $-18(1)$ & $-22(1)$ & $17(1)$ \\
\hline $\mathrm{C}(17)$ & $22(1)$ & $43(1)$ & $26(1)$ & $-7(1)$ & $0(1)$ & $9(1)$ \\
\hline $\mathrm{C}(18)$ & $32(1)$ & $68(1)$ & $40(1)$ & $-33(1)$ & $-9(1)$ & $16(1)$ \\
\hline $\mathrm{C}(19)$ & $50(1)$ & $115(2)$ & $78(1)$ & $-67(1)$ & $-22(1)$ & $44(1)$ \\
\hline $\mathrm{C}(20)$ & $60(1)$ & $105(1)$ & $120(2)$ & $-76(1)$ & $-41(1)$ & $59(1)$ \\
\hline
\end{tabular}




$\begin{array}{lllllll}\mathrm{C}(21) & 40(1) & 92(1) & 61(1) & -38(1) & -11(1) & 40(1) \\ \mathrm{C}(22) & 32(1) & 64(1) & 29(1) & -10(1) & -2(1) & 21(1) \\ \mathrm{C}(23) & 27(1) & 27(1) & 22(1) & 1(1) & -1(1) & -3(1) \\ \mathrm{C}(24) & 28(1) & 40(1) & 26(1) & 4(1) & -7(1) & -10(1) \\ \mathrm{C}(25) & 27(1) & 29(1) & 24(1) & 3(1) & -1(1) & -6(1) \\ \mathrm{C}(26) & 45(1) & 24(1) & 33(1) & -6(1) & -1(1) & -10(1) \\ \mathrm{C}(27) & 29(1) & 44(1) & 34(1) & 8(1) & -6(1) & -15(1) \\ \mathrm{C}(28) & 36(1) & 32(1) & 26(1) & 2(1) & 1(1) & -17(1) \\ \mathrm{C}(29) & 25(1) & 22(1) & 31(1) & -4(1) & 1(1) & 3(1) \\ \mathrm{C}(30) & 32(1) & 27(1) & 45(1) & 2(1) & -4(1) & 0(1) \\ \mathrm{C}(31) & 31(1) & 39(1) & 36(1) & -6(1) & 5(1) & -3(1) \\ \mathrm{C}(32) & 31(1) & 25(1) & 32(1) & -3(1) & -1(1) & 1(1) \\ \mathrm{C}(33) & 51(1) & 104(1) & 41(1) & 42(1) & 27(1) & 54(1) \\ \mathrm{C}\left(35^{\prime}\right) & 64(1) & 79(1) & 53(1) & -9(1) & 19(1) & -26(1) \\ \mathrm{C}(37) & 64(1) & 116(2) & 66(1) & -25(1) & 21(1) & -38(1) \\ \mathrm{C}(34) & 40(2) & 25(1) & 10(1) & -5(1) & -3(1) & -6(1) \\ \mathrm{C}(35) & 32(2) & 59(2) & 35(1) & 15(2) & -8(1) & 5(2) \\ \mathrm{C}(38) & 34(2) & 19(1) & 36(1) & 1(1) & -15(1) & 8(1) \\ \mathrm{C}\left(34^{\prime}\right) & 32(2) & 49(2) & 25(1) & 6(1) & -2(1) & -17(2) \\ \mathrm{C}\left(37^{\prime}\right) & 45(2) & 42(2) & 49(2) & 11(2) & 11(2) & -5(2) \\ \mathrm{C}\left(38^{\prime}\right) & 46(2) & 21(1) & 37(1) & -17(1) & -15(1) & 12(1) \\ & & & & & \end{array}$


Table S18. Hydrogen coordinates ( $\mathrm{x} 10^{4}$ ) and isotropic displacement parameters $\left(\AA^{2} \times 10^{3}\right)$ for 6 .

\begin{tabular}{|c|c|c|c|c|}
\hline & $\mathrm{x}$ & $\mathrm{y}$ & $\mathrm{Z}$ & $\mathrm{U}(\mathrm{eq})$ \\
\hline $\mathrm{H}(3 \mathrm{~A})$ & 2603 & 5437 & 6957 & 38 \\
\hline $\mathrm{H}(4 \mathrm{~A})$ & 1665 & 4858 & 6604 & 40 \\
\hline $\mathrm{H}(5 \mathrm{~A})$ & 909 & 5501 & 5883 & 37 \\
\hline $\mathrm{H}(6 \mathrm{~A})$ & 759 & 6700 & 7790 & 39 \\
\hline $\mathrm{H}(7 \mathrm{~A})$ & 1860 & 6732 & 8280 & 31 \\
\hline $\mathrm{H}(8 \mathrm{~A})$ & 2189 & 5726 & 8620 & 35 \\
\hline $\mathrm{H}(9 \mathrm{~A})$ & 1291 & 5072 & 8341 & 39 \\
\hline $\mathrm{H}(10 \mathrm{~A})$ & 408 & 5674 & 7828 & 51 \\
\hline $\mathrm{H}\left(6^{\prime} \mathrm{A}\right)$ & 376 & 6435 & 7516 & 53 \\
\hline $\mathrm{H}\left(7^{\prime} \mathrm{A}\right)$ & 1335 & 6962 & 7953 & 59 \\
\hline $\mathrm{H}\left(8^{\prime} \mathrm{A}\right)$ & 2043 & 6257 & 8657 & 61 \\
\hline H(9'A) & 1522 & 5296 & 8657 & 56 \\
\hline $\mathrm{H}(10 \mathrm{~B})$ & 492 & 5406 & 7952 & 44 \\
\hline $\mathrm{H}(11 \mathrm{~A})$ & 546 & 6758 & 4437 & 41 \\
\hline $\mathrm{H}(12 \mathrm{~A})$ & -16 & 6536 & 5589 & 52 \\
\hline $\mathrm{H}(12 \mathrm{~B})$ & 228 & 5911 & 5553 & 52 \\
\hline $\mathrm{H}(13 \mathrm{~A})$ & -481 & 6432 & 4308 & 66 \\
\hline $\mathrm{H}(13 \mathrm{~B})$ & -706 & 5942 & 4891 & 66 \\
\hline $\mathrm{H}(14 \mathrm{~A})$ & -39 & 5310 & 4272 & 89 \\
\hline $\mathrm{H}(14 \mathrm{~B})$ & -436 & 5590 & 3571 & 89 \\
\hline$H(15 A)$ & 596 & 5567 & 3192 & 73 \\
\hline $\mathrm{H}(15 \mathrm{~B})$ & 350 & 6190 & 3214 & 73 \\
\hline $\mathrm{H}(16 \mathrm{~A})$ & 1276 & 6180 & 3875 & 58 \\
\hline$H(16 B)$ & 1067 & 5683 & 4459 & 58 \\
\hline $\mathrm{H}(17 \mathrm{~A})$ & 680 & 7011 & 6520 & 36 \\
\hline $\mathrm{H}(18 \mathrm{~A})$ & 1634 & 7350 & 6884 & 56 \\
\hline $\mathrm{H}(18 \mathrm{~B})$ & 1622 & 7786 & 6149 & 56 \\
\hline $\mathrm{H}(19 \mathrm{~A})$ & 1401 & 8266 & 7349 & 97 \\
\hline H(19B) & 882 & 7835 & 7574 & 97 \\
\hline
\end{tabular}




\begin{tabular}{|c|c|c|c|c|}
\hline $\mathrm{H}(20 \mathrm{~A})$ & 438 & 8636 & 7027 & 114 \\
\hline $\mathrm{H}(20 \mathrm{~B})$ & 852 & 8602 & 6233 & 114 \\
\hline $\mathrm{H}(21 \mathrm{~A})$ & -94 & 8277 & 5921 & 78 \\
\hline $\mathrm{H}(21 \mathrm{~B})$ & -59 & 7834 & 6646 & 78 \\
\hline $\mathrm{H}(22 \mathrm{~A})$ & 666 & 7805 & 5198 & 50 \\
\hline $\mathrm{H}(22 \mathrm{~B})$ & 139 & 7378 & 5400 & 50 \\
\hline $\mathrm{H}(23 \mathrm{~A})$ & 2388 & 6919 & 6602 & 30 \\
\hline $\mathrm{H}(24 \mathrm{~A})$ & 2848 & 6409 & 7639 & 47 \\
\hline $\mathrm{H}(24 \mathrm{~B})$ & 3320 & 6805 & 7195 & 47 \\
\hline $\mathrm{H}(24 \mathrm{C})$ & 3311 & 6155 & 6997 & 47 \\
\hline $\mathrm{H}(26 \mathrm{~A})$ & 3465 & 8029 & 6200 & 51 \\
\hline $\mathrm{H}(26 \mathrm{~B})$ & 3074 & 7551 & 6619 & 51 \\
\hline $\mathrm{H}(26 \mathrm{C})$ & 2818 & 7882 & 5848 & 51 \\
\hline $\mathrm{H}(27 \mathrm{~A})$ & 4331 & 7418 & 5934 & 53 \\
\hline $\mathrm{H}(27 \mathrm{~B})$ & 4242 & 6832 & 5491 & 53 \\
\hline $\mathrm{H}(27 \mathrm{C})$ & 4000 & 6922 & 6400 & 53 \\
\hline $\mathrm{H}(28 \mathrm{~A})$ & 3884 & 7892 & 4807 & 47 \\
\hline $\mathrm{H}(28 \mathrm{~B})$ & 3216 & 7769 & 4511 & 47 \\
\hline $\mathrm{H}(28 \mathrm{C})$ & 3738 & 7336 & 4314 & 47 \\
\hline $\mathrm{H}(30 \mathrm{~A})$ & 3763 & 5416 & 5253 & 52 \\
\hline $\mathrm{H}(30 \mathrm{~B})$ & 3343 & 5624 & 5981 & 52 \\
\hline $\mathrm{H}(30 \mathrm{C})$ & 3925 & 5972 & 5739 & 52 \\
\hline $\mathrm{H}(31 \mathrm{~A})$ & 3810 & 5895 & 3975 & 53 \\
\hline $\mathrm{H}(31 \mathrm{~B})$ & 3948 & 6483 & 4388 & 53 \\
\hline $\mathrm{H}(31 \mathrm{C})$ & 3394 & 6418 & 3779 & 53 \\
\hline $\mathrm{H}(32 \mathrm{~A})$ & 2863 & 5392 & 4337 & 44 \\
\hline $\mathrm{H}(32 \mathrm{~B})$ & 2500 & 5950 & 4156 & 44 \\
\hline $\mathrm{H}(32 \mathrm{C})$ & 2430 & 5645 & 5018 & 44 \\
\hline $\mathrm{H}(34 \mathrm{~A})$ & 987 & 6929 & 3254 & 30 \\
\hline $\mathrm{H}(35 \mathrm{~A})$ & 269 & 7580 & 2656 & 50 \\
\hline $\mathrm{H}(38 \mathrm{~A})$ & 1603 & 8202 & 4620 & 36 \\
\hline $\mathrm{H}(34 \mathrm{~B})$ & 959 & 7262 & 3307 & 42 \\
\hline $\mathrm{H}(37 \mathrm{~A})$ & 1388 & 9046 & 4360 & 55 \\
\hline $\mathrm{H}(38 \mathrm{~B})$ & 1966 & 8251 & 4795 & 42 \\
\hline H(39A) & 2983 & 4473 & 8973 & 182 \\
\hline $\mathrm{H}(39 \mathrm{~B})$ & 2878 & 3811 & 8928 & 182 \\
\hline
\end{tabular}




$\begin{array}{lrrrr}\mathrm{H}(40 \mathrm{~A}) & 2502 & 4628 & 7868 & 189 \\ \mathrm{H}(40 B) & 2741 & 4019 & 7660 & 189 \\ \mathrm{H}(41 \mathrm{~A}) & 1711 & 4261 & 7507 & 210 \\ \mathrm{H}(41 \mathrm{~B}) & 1906 & 3654 & 7827 & 210 \\ \mathrm{H}(42 \mathrm{~A}) & 1413 & 3858 & 8793 & 87 \\ \mathrm{H}(42 B) & 1445 & 4505 & 8597 & 87 \\ \mathrm{H}(43 \mathrm{~A}) & 4319 & 4944 & 6732 & 474 \\ \mathrm{H}(43 \mathrm{~B}) & 4752 & 5320 & 7241 & 474 \\ \mathrm{H}(43 \mathrm{C}) & 4840 & 4675 & 7234 & 474 \\ \mathrm{H}(44 \mathrm{~A}) & 3904 & 4619 & 8011 & 339 \\ \mathrm{H}(44 \mathrm{~B}) & 3840 & 5288 & 7932 & 339 \\ \mathrm{H}(45 \mathrm{~A}) & 5055 & 4826 & 8588 & 285 \\ \mathrm{H}(45 B) & 4841 & 5455 & 8732 & 285 \\ \mathrm{H}(46 \mathrm{~A}) & 4289 & 4542 & 9529 & 453 \\ \mathrm{H}(46 \mathrm{~B}) & 4148 & 5175 & 9588 & 453 \\ \mathrm{H}(47 \mathrm{~A}) & 4717 & 4947 & 10542 & 500 \\ \mathrm{H}(47 \mathrm{~B}) & 5176 & 4731 & 9894 & 500 \\ \mathrm{H}(47 \mathrm{C}) & 5034 & 5367 & 9953 & 500\end{array}$

